\title{
A Comprehensive Spectroscopic Analysis of DB White Dwarfs
}

\author{
P. Bergeron ${ }^{1}$, F. Wesemael ${ }^{1}$, Pierre Dufour ${ }^{1}$, A. Beauchamp ${ }^{1,2}$, C. Hunter ${ }^{1,3}$, Rex A. \\ Saffer $^{4}$, A. Gianninas ${ }^{1}$, M. T. Ruiz ${ }^{5}$, M.-M. Limoges ${ }^{1}$, Patrick Dufour ${ }^{1}$, G. Fontaine ${ }^{1}$, \\ James Liebert ${ }^{6}$ \\ ${ }^{1}$ Département de Physique, Université de Montréal, C.P. 6128, Succ. Centre-Ville, \\ Montréal, QC H3C 3J\%, Canada; bergeron@astro.umontreal.ca, \\ wesemael@astro.umontreal.ca,gianninas@astro.umontreal.ca,limoges@astro.umontreal.ca, \\ dufourpa@astro.umontreal.ca,fontaine@astro.umontreal.ca \\ ${ }^{2}$ Forensic Technologies Wai Inc., 5757 Boulevard Cavendish, Montréal, QC H4W 2W8, \\ Canada; alain.beauchamp@fti-ibis.com \\ ${ }^{3}$ ITS Yale University, Box 27387, West Haven, CT 06516, USA; chris.hunter@yale.edu \\ ${ }^{4}$ DataTime Consulting, 109 Forrest Avenue, Narberth, PA 19072, USA; \\ rex.saffer@comcast.net \\ ${ }^{5}$ Departamento de Astronomía, Universidad de Chile, Casilla 36-D, Santiago, Chile; \\ mtruiz@das.uchile.cl \\ ${ }^{6}$ Steward Observatory, University of Arizona, Tucson, AZ 85721, USA
}

\begin{abstract}
We present a detailed analysis of 108 helium-line (DB) white dwarfs based on model atmosphere fits to high signal-to-noise optical spectroscopy. We derive a mean mass of $0.67 M_{\odot}$ for our sample, with a dispersion of only $0.09 M_{\odot}$. White dwarfs also showing hydrogen lines, the DBA stars, comprise $44 \%$ of our sample, and their mass distribution appears similar to that of DB stars. As in our previous investigation, we find no evidence for the existence of low-mass $\left(M<0.5 M_{\odot}\right)$ DB white dwarfs. We derive a luminosity function based on a subset of DB white dwarfs identified in the Palomar-Green survey. We show that $20 \%$ of all white dwarfs in the temperature range of interest are DB stars, although the fraction drops to half this value above $T_{\text {eff }} \sim 20,000 \mathrm{~K}$. We also show that the persistence of DB stars with no hydrogen features at low temperatures is difficult to reconcile with a scenario involving accretion from the interstellar medium, often invoked to account for the observed hydrogen abundances in DBA stars. We present evidence for the existence of two different evolutionary channels that produce
\end{abstract}


DB white dwarfs: the standard model where DA stars are transformed into DB stars through the convective dilution of a thin hydrogen layer, and a second channel where DB stars retain a helium-atmosphere throughout their evolution. We finally demonstrate that the instability strip of pulsating V777 Her white dwarfs contains no nonvariables, if the hydrogen content of these stars is properly accounted for.

Subject headings: stars: abundances — stars: evolution — stars: fundamental parameters — stars: luminosity function, mass function — stars: oscillations white dwarfs

\section{Introduction}

DB white dwarfs are characterized by the extreme purity of their helium-dominated atmospheres with, occasionally, only minute traces of hydrogen (DBA stars) and heavy elements (DBQ or DBZ stars) seen in their optical spectra. It has been 15 years since we last reviewed the work carried out in Montreal on the optical spectra of DB and DBA white dwarfs (Beauchamp et al. 1996). Parts of this work have been published over the years Beauchamp et al. 1995, 1997; Beauchamp 1998; Beauchamp \& Wesemael 1998; Beauchamp et al. 1999; Hunter et al. 2001; Wesemael et al. 2001), and full and definitive results from this longterm project are presented here.

Progress on several fronts relevant to the study of DB stars has been significant since our review (Beauchamp et al. 1996): the discovery of stars in the DB gap through the Sloan Digital Sky Survey (SDSS; Eisenstein et al. 2006), the discovery of a new class of carbon-rich stars - the so-called Hot DQ stars (Dufour et al. 2007b, 2008), the analysis of the DB stars in the ESO Supernova Ia Progenitor Survey (SPY; Voss et al. 2007), the far-ultraviolet observations of DB stars with FUSE (Petitclerc et al. 2005; Desharnais et al. 2008; Dufour et al. 2010a), the likelihood of atmospheric contamination of the photospheres of DB stars through disk-fed accretion (e.g., Dufour et al. 2010b), etc. Yet our knowledge of some of the fundamental properties of DB stars, embodied in a set of questions posed by Beauchamp et al. (1996), remains sketchy. These questions referred to the respective mass distributions for the DA and DB stars and to the possible existence of a bimodal mass distribution for DB degenerates, to the existence of statistical evidence for differences between the DB and DBA samples, as well as to the existence of atmospheric peculiarities in hot DB stars that might afford possible evidence for a recent episode of convective mixing.

One particular problem with the analysis of DB white dwarfs is related to the effect of 
invisible traces of hydrogen that can affect significantly the temperature scale of DB stars based on line profile fitting techniques. For instance, Beauchamp et al. (1999, see their Table 1) showed that small amounts of hydrogen, of the order of $\mathrm{H} / \mathrm{He} \sim 10^{-5}-10^{-4}$, can produce differences as large as $3000 \mathrm{~K}$ in $T_{\text {eff }}$ for the hottest DB stars. In the case of the Beauchamp et al. analysis, upper limits on the hydrogen abundance were based only on the absence of $\mathrm{H} \beta$. To improve on this aspect of the analysis, Voss et al. (2007) secured high-resolution spectra in the region near $\mathrm{H} \alpha$ for a sample of $\sim 60$ DB stars in the SPY survey. A similar analysis had been carried out earlier by Hunter et al. (2001), but on a smaller sample of hot DB stars. With their high-resolution data, Voss et al. were able to secure more stringent limits on the hydrogen abundance. In their Table 1, they contrast the effective temperatures and surface gravities of DB stars without visible hydrogen obtained from two distinct grids of model atmospheres: one with a pure helium composition, and one with a trace of hydrogen, $\mathrm{H} / \mathrm{He}=10^{-5}$, the upper limit imposed by their data. As discussed in Voss et al., the average temperature difference they measure, around $230 \mathrm{~K}$, is significantly smaller than that obtained by Beauchamp et al. (1999) based on much higher upper limits on the hydrogen abundance. However, this evaluation is somewhat misleading since the upper limit imposed by the SPY data is strongly temperature dependent according to the results shown in Figure 9 of Voss et al. In fact, at $T_{\text {eff }} \sim 24,000 \mathrm{~K}$, their detection limit of $300 \mathrm{~m} \AA$ for the $\mathrm{H} \alpha$ equivalent width indicates an upper limit of $\mathrm{H} / \mathrm{He} \sim 10^{-4}$, or a factor of ten larger than the value used in their Table 1. Hence we feel that the influence of invisible amounts of hydrogen on the determination of the atmospheric parameters of DB stars still needs to be properly addressed.

Another complication with the spectroscopic analysis of DB stars is related to the problem of the convective efficiency in their atmospheres. While the spectroscopic analysis of Beauchamp et al. (1999) relied on model atmospheres calculated with the so-called ML2/ $\alpha=1.25$ version of the mixing-length theory, Voss et al. (2007) adopted in their study a much less efficient $\alpha=0.6$ version, i.e. the same convective efficiency as for DA white dwarfs (Bergeron et al. 1995). This may represent an important source of discrepancy when comparing the atmospheric parameters from various analyses. While some authors argued that there is no reason to expect the mixing length parameterization to be different in DA and DB white dwarfs (e.g., Castanheira et al. 2006), we may as well argue that there is no reason to expect it to be identical. Hence we feel the problem of the convective efficiency in the atmospheres of DB stars needs to be reexamined as well.

Of deeper astrophysical interest is the question of the possible evolutionary channels that could produce DB white dwarfs. The progenitors of DB white dwarfs are believed to be DA stars, transformed into helium-atmosphere white dwarfs as a result of the convective dilution of a thin radiative hydrogen layer $\left(M_{\mathrm{H}} \sim 10^{-15} M_{\odot}\right)$ with the deeper convective 
helium envelope (Fontaine \& Wesemael 1987). Evidence for this mechanism rests on the complete absence of helium-atmosphere white dwarfs in the Palomar-Green (PG) survey between $T_{\text {eff }} \sim 45,000 \mathrm{~K}$ - where the coolest DO stars are found, and $\sim 30,000 \mathrm{~K}-$ where the hottest DB stars are located. This so-called DB gap has partially been filled in by the discovery of extremely hot DB stars in the SDSS (Eisenstein et al. 2006), although the number of DB stars in the gap remains a factor of 2.5 lower than what is expected based on the number of cooler DB stars in the SDSS. We are thus dealing with a DB deficiency, rather than with a real gap, in this particular range of effective temperature. How do these DB stars in the gap fit in our understanding of white dwarf evolution in general?

All these questions remain current, and need to be addressed for a complete understanding of the physical properties and evolutionary status of DB stars. With the hope of shedding some light on these issues, we present in this paper a complete and comprehensive spectroscopic analysis of over 100 DB stars. We first begin in Section 2 with a theoretical investigation of model atmospheres of DB stars to explore the observational and theoretical issues at stake. The observational data used in our analysis are then presented in Section 3. Our spectroscopic analysis follows in Section 4 and the results are interpreted in Section 5. We present our conclusions in Section 6.

\section{Theoretical Considerations}

\subsection{Model Atmospheres}

Our model atmospheres and synthetic spectra are built from the model atmosphere code described in Tremblay \& Bergeron (2009) and references therein, in which we have incorporated the improved Stark profiles of neutral helium of Beauchamp et al. (1997). These detailed profiles for over twenty lines of neutral helium take into account the transition from quadratic to linear Stark broadening, the transition from impact to quasi-static regime for electrons, as well as forbidden components. In the context of DB stars, these models are comparable to those described by Beauchamp (1995) and Beauchamp et al. (1996), with the exception that at low temperatures $\left(T_{\text {eff }}<10,800 \mathrm{~K}\right)$, we now use the free-free absorption coefficient of the negative helium ion of John (1994). For the hydrogen lines in DBA stars, we rely on the improved calculations for Stark broadening of Tremblay \& Bergeron (2009). Our models are in LTE and include convective energy transport treated within the mixinglength theory. The theoretical spectra are calculated using the occupation formalism of Hummer \& Mihalas (1988) for both the hydrogen and helium populations and corresponding bound-bound, bound-free, and pseudo-continuum opacities. 
An important issue related to the modeling of DB spectra is the inclusion of van der Waals broadening at low effective temperatures $\left(T_{\text {eff }} \lesssim 15,000 \mathrm{~K}\right)$, where Stark broadening is no longer the dominant source of line broadening. Beauchamp et al. (1996, see their Figure 6 ) show the effect of this broadening mechanism in a $\log g$ vs $T_{\text {eff }}$ diagram for DB stars with parameters determined from optical spectroscopy. The neglect of van der Waals broadening results in spuriously high $\log g$ values at low effective temperatures. In this series of synthetic spectrum calculations, we include the treatment of this broadening mechanism provided by Deridder \& Van Rensbergen (1976).

Our model grid covers a range of effective temperature between $T_{\text {eff }}=11,000 \mathrm{~K}$ and $40,000 \mathrm{~K}$ by steps of $1000 \mathrm{~K}$, while the $\log g$ ranges from 7.0 to 9.0 by steps of 0.5 dex. In addition to pure helium models, we also calculated models with $\log \mathrm{H} / \mathrm{He}=-6.5$ to -2.0 by steps of 0.5 . In order to explore the effect of varying the convective efficiency on the predicted emergent fluxes, models have been calculated with the ML2 version of the mixing-length theory, which corresponds to the prescription of Böhm \& Cassinelli (1971), with various values of $\alpha=\ell / H$, i.e. the ratio of the mixing length to the pressure scale height, ranging from 0.75 to 1.75 by steps of 0.25 .

\subsection{Exploration of the Parameter Space}

The determination of the atmospheric parameters, $T_{\text {eff }}, \log g$, and $\mathrm{H} / \mathrm{He}$, of DB stars represents an inherent difficulty in any analysis of their properties. While the atmospheric parameters for DA stars derived from various studies agree generally well (see, e.g., Figure 9 of Liebert et al. 2005), a similar comparison for DB stars is less than satisfactory, as shown for instance in Figures 3 and 4 of Voss et al. (2007), which compare the values obtained from the SPY survey with those obtained from independent studies (Beauchamp et al. 1999; Friedrich et al. 2000; Castanheira et al. 2006).

In addition to being rarer than their hydrogen-line DA counterparts, the hotter DB stars are characterized by an optical spectrum where the neutral helium transitions exhibit little sensitivity to effective temperature. As an illustration, we show in the left panel of Figure 1 the model spectra 1 for various values of $T_{\text {eff }}$ (shown in blue), compared to a model template at $T_{\text {eff }}=24,000 \mathrm{~K}$ (shown in red). There is a wide range of temperature, between $T_{\text {eff }} \sim 20,000 \mathrm{~K}$ and 30,000 K, where the optical spectra are virtually identical. This underscores the need to secure well calibrated, high signal-to-noise spectroscopic observations to study these stars. Equivalently, we show in the left panel of Figure 2 the variation of the

\footnotetext{
${ }^{1}$ Unless specified, the models displayed here assume a value of $\alpha=1.25$.
} 
equivalent width of He I $\lambda 4471$ as a function of effective temperature for various hydrogen abundances. In all cases, the variation of the equivalent width shows a wide plateau, about $10,000 \mathrm{~K}$ wide, that inhibits the determination of accurate effective temperatures from the optical spectrum of DB stars in this particular range of temperature. Also shown in this figure are the measured equivalent widths in our sample of DB stars, arbitrarily located at $T_{\text {eff }}=22,000 \mathrm{~K}$. Interestingly enough, the DB stars in our sample with the strongest lines have equivalent widths that are larger than predicted by any of our models shown in this panel.

Luckily, we fare somewhat better with gravity indicators, as illustrated in the middle panel of Figure1, The large gravity-sensitivity of several segments containing neutral helium transitions (notably the $2^{3} P-6^{3} D \lambda 3819$ line, the $4100-4200 \AA$ region that contains the $2^{3} P-5^{3} S \lambda 4121,2^{1} P-6^{1} D \lambda 4144$, and $2^{1} P-6^{1} S \lambda 4169$ lines, as well as the strong $2^{1} P-5^{1} D \lambda 4388$ transition; Wickramasinghe 1979) can be used successfully through the entire temperature range. We disagree in that respect with the statement of Voss et al. (2007, see Section 3.1) that the dependence of line profiles on $\log g$ is smaller for the cooler DB stars. Interestingly, the variation of the He I $\lambda 4471$ equivalent width with $T_{\text {eff }}$ is also strongly $\log g$ dependent, as can be observed in the middle panel of Figure 2, For low-gravity DB white dwarfs, the maximum is now strongly peaked near 22,000 K, while for high-gravity DB stars, the plateau becomes even wider, and the maximum equivalent width is reached at temperatures near 33,000 K. Again, variations of $\log g$ help very little in matching the maximum observed equivalent widths of the DB stars in our sample.

Less well documented is the sensitivity of the optical spectrum of DB stars to the convective efficiency. Helium atmospheres of white dwarfs become convective below $T_{\text {eff }} \sim$ $60,000 \mathrm{~K}$, but it is not until the temperature drops below $\sim 30,000 \mathrm{~K}$ that the helium convection zone becomes sufficiently important, depending on the assumed convective efficiency (see Figure 10 of Tassoul et al. 1990). For completeness and future reference, we show in Figure 3 the extent of the helium convection zone in a $0.6 M_{\odot}$ DB white dwarf. Convective energy transport is generally treated in model atmosphere calculations within the mixing-length theory, and unless convection becomes adiabatic, the emergent model fluxes will depend on the assumed convective efficiency, parameterized in this case by the free parameter $\alpha$. This additional parameterization was first introduced in the context of DB stars by Beauchamp (1995) on the basis of work carried out in the hydrogen-line DA stars (Bergeron et al. 1995).

To illustrate this sensitivity, we compare in the right panel of Figure 1 model spectra calculated with $\alpha=0.75$ and 1.75. The effect is subtle, and only noticeable between $T_{\text {eff }} \sim$ $18,000 \mathrm{~K}$ and $28,000 \mathrm{~K}$. At higher effective temperatures, the atmospheres of DB stars 
become almost completely radiative (see Figure 3), while at lower temperatures convection becomes adiabatic, and the convective efficiency no longer depends on the specific value of $\alpha$. Despite the apparent lack of sensitivity to variations of $\alpha$, the effects on the normalized line profiles are more pronounced. This is illustrated in the right panel of Figure 2, where the equivalent width of He I $\lambda 4471$ as a function of effective temperature is shown for model atmospheres calculated with various convective efficiencies. Once again, the line strength is sensitive to $\alpha$ for effective temperatures between $18,000 \mathrm{~K}$ and $28,000 \mathrm{~K}$ and, in contrast to the results shown in the previous panels, the largest predicted equivalent widths (achieved with $\alpha=0.75)$ are larger than the largest measured values. This means that, were we to overestimate $\alpha$ in our models, all the stars would be shifted toward the maximum of the curve (i.e., near 30,000 $\mathrm{K}$ for $\alpha=1.75$ ) and form a clump. In contrast, were we to underestimate $\alpha$, the stars we fit would be lumped on respective sides of the maximum of the curve (i.e., near 18,000 $\mathrm{K}$ and 30,000 $\mathrm{K}$ for $\alpha=0.75$ ), and gaps would develop in the distribution of stars with respect to effective temperature. This behavior can be used to constrain $\alpha$, as we show below.

We finally note that the results presented here are consistent with those of Voss et al. (2007) who mention that the helium lines in their models, calculated with ML2/ $\alpha=0.6$ (i.e. a value even smaller than the smallest value used in Figure 2), reach a maximum strength around $T_{\text {eff }}=20,000 \mathrm{~K}$.

\section{Spectroscopic Observations}

Our sample of DB stars was selected from the online version of the Villanova White Dwarf Catalog2. One of the first goals of this project was to secure spectroscopic observations of all DB stars identified in the PG Survey (Green et al. 1986) in order to compare the luminosity function of DB stars with that obtained by Liebert et al. (2005) for DA stars. Otherwise, DB white dwarfs were routinely observed over the years and included in our sample. Due to observational constraints, we focused mainly on the brightest white dwarfs in the catalog, and as such, there is little overlap between our sample and the SDSS sample. The distribution of $V$ magnitudes 3 for the 118 objects observed spectroscopically is presented in Figure 4 (we exclude here the 10 misclassified stars discussed further below). Several objects in this original sample are excluded from our analysis. Three are DA + DB double degenerate systems and have been analyzed elsewhere: PG 1115+166 (Bergeron \& Liebert 2002), KUV

\footnotetext{
${ }^{2}$ http://www.astronomy.villanova.edu/WDCatalog/index.html

${ }^{3}$ These can be photographic magnitudes in some cases, or even $B$ magnitudes.
} 
02196+2816 (Limoges et al. 2009), and KUV 14197+2514 (Limoges \& Bergeron 2010). Two are magnetic DB stars: PG 0853+164 (Wesemael et al. 2001) and Feige 7 (Achilleos et al. 1992). Finally, five white dwarfs do not show enough helium lines in their optical spectra to be analyzed quantitatively: LP 891-12 (0443-275.1), G102-39 (0551+123), GD 84 (0714+458), G227-5 (1727+560), and LDS 678A (1917-077).

We are thus left with a total of 108 DB white dwarfs, including several DBA and DBZ stars (the analysis of Beauchamp et al. (1996) included close to 80 objects). The complete list of objects is provided in Table 1. For comparison, a recent analysis based on the SPY sample (Voss et al. 2007), the largest completed up to now, includes 69 white dwarfs. Most of our objects were observed at high signal-to-noise ratio $(\mathrm{S} / \mathrm{N})$ in the blue region of the optical spectrum (3700-5200 ^) with the Steward Observatory $2.3 \mathrm{~m}$ Bok Telescope equipped with the Boller \& Chivens spectrograph. The 4 .'5 slit together with the 600 line $\mathrm{mm}^{-1}$ grating blazed at $3568 \AA$ in first order provides a spectral coverage from about 3500 to $5250 \AA$ at a resolution of $\sim 6 \AA$ FWHM. Additional blue spectra in the southern hemisphere were secured at Carnegie Observatories' 2.5 m Irénée du Pont Telescope at Las Campanas (Chile) with the Boller \& Chivens spectrograph. The $1^{\prime \prime} .5$ slit with the 600 line $\mathrm{mm}^{-1}$ grating blazed at 5000 $\AA$ provided a spectral coverage from about 3500 to $6600 \AA$ at a slightly better resolution of $3 \AA$ FWHM. Finally, the spectrum of GD $554(2250+746)$ was obtained at the Mt. Hopkins $6.5 \mathrm{~m}$ MMT telescope using the Blue Channel of the MMT Spectrograph. The 500 lines $\mathrm{mm}^{-1}$ grating and a $1^{\prime \prime} .0$ slit provided a spectral coverage from about 3400 to $6300 \AA$ with a resolution of $\sim 4 \AA \mathrm{FWHM}$.

Our blue optical spectra are displayed in Figure 5 in order of decreasing effective temperature. In addition to the numerous neutral helium lines that dominate the spectra of these stars, hydrogen lines $(\mathrm{H} \beta)$ and the Ca II $\mathrm{H} \& \mathrm{~K}$ doublet are also visible in some objects. The spectrum of L7-44 (1708-871) suffers from residual flux calibration problem, while that of HE 0429-1651 (0429-168) is significantly contaminated by the presence of an M dwarf companion. Note that the spectra of the DB stars recently identified by Lépine et al. (2011) represent new observations at higher $\mathrm{S} / \mathrm{N}$. One of the most obvious qualities of our sample is its homogeneity, both in terms of wavelength coverage and signal-to-noise. While its size remains small compared to that of the SDSS sample, there is no comparison in terms of the quality of the spectra, as can be seen from Figure 6, where we compare the distribution of S/N of our observations with that of the SDSS spectra from the Data Release 4. The majority of our spectra have S/N well above 50, while the SDSS spectra are strongly peaked between $\mathrm{S} / \mathrm{N} \sim 5$ and 20. Since the exposure time of a given SDSS spectrum is set by that of the entire plate, the corresponding $\mathrm{S} / \mathrm{N}$ is necessarily a function of the magnitude of the star, and SDSS white dwarfs are much fainter than those studied here. 
As part of our survey, we observed several DB white dwarf candidates that turned out to be lower surface gravity objects, mostly sdOB stars (with the glaring exception of one DA star). For completeness, we show these misclassified objects in Figure 7, Note that several of these have already been reclassified properly in the online version of the Villanova White Dwarf Catalog.

Because coverage of the $\mathrm{H} \alpha$ line is essential for the determination of accurate atmospheric parameters for DB stars, we complemented our blue data with red spectra obtained from several distinct sources: the KPNO $4 \mathrm{~m}$ data used by Hunter et al. (2001), spectra secured at the du Pont $2.5 \mathrm{~m}$ telescope, spectra extracted from the Data Release 4 of the SDSS, and SPY spectra from Voss et al. (2007, only those with $\mathrm{H} \alpha$ detected), available from the ESO archive web site. We have also recently secured additional spectra at the Steward Observatory $2.3 \mathrm{~m}$ Bok Telescope and at the KPNO $4 \mathrm{~m}$ telescope. Our own spectroscopic observations (36 objects) as well as SDSS spectra (17 objects) are displayed in Figure 8 in order of right ascension; these have typically 3 to $5 \AA$ resolution (FWHM). SPY/UVES spectra for 13 additional objects can be visualized as online material in Voss et al. (2007). To guide the eye, we also show in this figure the location of the $\mathrm{H} \alpha$ absorption feature. Hydrogen is detected in $50 \%$ of the objects shown here, a value in good agreement with the $55 \%$ fraction of DBA stars estimated by Voss et al. (2007). This fraction drops to $\sim 44 \%$ if we consider all stars in our sample, since 42 objects remain without $\mathrm{H} \alpha$ data. Note how the strength of $\mathrm{H} \alpha$ varies considerably from object to object, and how it is particularly strong in LP 497-114 (1311+129).

Finally, in Section 4.4, we also make use of IUE low-dispersion spectra issued from the re-

duction procedure of Holberg et al. (2003), which follows the prescription of Massa \& Fitzpatrick (2000) for the correction of residual temporal and thermal effects and for the absolute flux calibration. A total of 34 DB stars in our sample have UV spectra suitable for analysis.

\section{Atmospheric Parameter Determination}

\subsection{Fitting Technique}

Our fitting technique is similar to that outlined in Liebert et al. (2005, and references therein) for the analysis of DA stars. The first step is to normalize the flux from individual predefined wavelength segments, in both observed and model spectra, to a continuum set to unity. The comparison with model spectra, which are convolved with the appropriate Gaussian instrumental profile, is then carried out in terms of this normalized spectrum only. The most sensitive aspect of this approach is to properly define the continuum of the observed 
spectra. Here we rely on the procedure developed by Liebert et al. (2005), where the fluxed spectrum is first fitted with model spectra, multiplied by a high order polynomial (up to $\lambda^{5}$ ), in order to account for any residual error in the flux calibration. The nonlinear least-squares minimization method of Levenberg-Marquardt (Press et al. 1986) is used throughout. Note that the values of $T_{\text {eff }}$ and $\log g$ derived in this first step are meaningless since too many fitting parameters are considered, and the model fit just serves as a smooth fitting function used to define the continuum of the observed spectrum. Normal points are then fixed at several wavelengths defined by this smooth function, and used to normalize the spectrum. This method turns out to be quite accurate when a glitch is present in the spectrum at the location where the continuum is set. It also provides a precise value of the line center, which can be corrected to the laboratory wavelength. An example of continuum fitting using this procedure is illustrated in the top panel of Figure 9.

Once the spectrum is normalized to a continuum set to unity, as shown in the bottom panel of Figure 9, we use our grid of model spectra to determine $T_{\text {eff }}, \log g$, and $\mathrm{H} / \mathrm{He}$ in terms of the normalized spectrum only. Our three-dimensional minimization technique again relies on the nonlinear least-squares method of Levenberg-Marquardt, which is based on a steepest descent method.

\subsection{Determination of $T_{\text {eff }}, \log g$, and $\mathbf{H} / \mathbf{H e}$}

As shown in Figure 2, two solutions exist for a given DB spectrum, one on each side of the maximum strength of the neutral helium lines. In most cases, it is easy to distinguish these cool and hot solutions from an examination of our best fits, as displayed in the left panel of Figure 10 for the cool DB star LP 475-242 (0437+138). In addition, we also look at our spectroscopic solution in terms of absolute fluxes (not shown here) by normalizing both the observed and predicted model spectra at $4250 \AA$. In general, the slopes agree well, except in some cases where the object has been observed at high airmasses, in which case the slope of the observed spectrum might be distorted.

However, when the star is close to the temperature where the helium lines reach their maximum strength - a temperature range that strongly depends on $\log g$ and on the assumed convective efficiency according to Figure 2 - the cool and hot solutions cannot be distinguished, as shown in the right panel of Figure 10 for the hot DB star PG 1115+158. The comparison of the slopes of the energy distributions does not help either in this case. Fortunately, we discovered that this ambiguity could be resolved with the help of spectroscopic observations at $\mathrm{H} \alpha$, which add an additional constraint in our fitting procedure. Since our $\mathrm{H} \alpha$ data are independent of our blue observations, we use an iterative procedure where 
we fit the blue spectrum with an assumed hydrogen abundance to obtain a first estimate of $T_{\text {eff }}$ and $\log g$. The $\mathrm{H} \alpha$ spectra are then used to determine the hydrogen abundance, or upper limits on $\mathrm{H} / \mathrm{He}$, at these particular values of $T_{\text {eff }}$ and $\log g$. The procedure is then repeated until the value of $\mathrm{H} / \mathrm{He}$ has converged. An example of our solution for PG 1115+158 is displayed in Figure 11. The weak $\mathrm{H} \alpha$ absorption feature shown in the insert (from a SDSS spectrum, in this case) serves as an important constraint to determine the hydrogen abundance, which otherwise could only be inferred from the weak depression observed near $\mathrm{H} \beta$. Interestingly, our final solution for this object lies between the cool and hot solutions displayed in Figure 10 .

The procedure described above works well when any hydrogen absorption feature (H $\alpha$ or $\mathrm{H} \beta$ ) is visible in the optical spectrum. When no such feature is present, only upper limits on the hydrogen abundance can be determined. These limits depend on the signal-to-noise ratio of the observations, and on whether they are based on our blue spectra for $\mathrm{H} \beta$, or our red spectra for $\mathrm{H} \alpha$. We show, in Figure 12, the limits on the hydrogen abundance imposed by the absence of $\mathrm{H} \alpha$ and $\mathrm{H} \beta$ features in our spectra (we adopt a detection limit of 200 $\mathrm{m} \AA$ and $300 \mathrm{~m} \AA$ for the equivalent widths of $\mathrm{H} \alpha$ and $\mathrm{H} \beta$, respectively); the results for $\mathrm{H} \alpha$ are qualitatively similar to those shown in Figure 9 of Voss et al. (2007). For high S/N spectra with no detectable hydrogen feature4, we set the hydrogen abundance in our fitting procedure at the value given by these upper limits at the appropriate temperature. In cases where the spectrum is noisier, our fitting procedure may find an upper limit on the hydrogen abundance that is larger than those of Figure 12.

\subsection{DBZ White Dwarfs}

Four DBZ stars in our sample have sufficiently strong calcium lines that the inclusion of the Ca II H \& K doublet is required to fit them properly. These are GD 40 (0300-013), CBS 78 (0838+375), KUV 15519+1730 (1551+175), and G241-6 (2222+683). We take a simple approach here of including only calcium in our equation of state and opacity calculations. A more detailed analysis of these DBZ stars, including additional heavy elements, will be presented elsewhere. A smaller grid of models between $T_{\text {eff }}=12,000 \mathrm{~K}$ and $17,000 \mathrm{~K}$ has been calculated for this purpose, with calcium abundances of $\log \mathrm{Ca} / \mathrm{He}=-7.5$ to -6.0 by steps of 0.5 , and the same $\log g$ and $\mathrm{H} / \mathrm{He}$ values as above. For each star we adopt the

\footnotetext{
${ }^{4}$ We use in particular the fact that no detectable $\mathrm{H} \alpha$ absorption feature has been reported by Voss et al. (2007, see their Table 2) in 0308-565, 0429-168, 0845-188, 0900+142, 1046-017, 1144-084, 1336+123, $2129+000$, and 2144-079.
} 
calcium abundance that best reproduces the calcium doublet. Our best fits for these 4 DBZ stars are presented in Figure 13.

\subsection{Convective Efficiency in DB White Dwarfs}

The problem of the convective efficiency in the atmosphere of DA stars has been tackled by Bergeron et al. (1995) who used optical spectroscopic observations combined with UV energy distributions to show that the so-called ML2 $/ \alpha=0.6$ parametrization of the mixing-length theory provides the best internal consistency between optical and UV effective temperatures, trigonometric parallaxes, $V$ magnitudes, and gravitational redshift:5. Similarly, Beauchamp et al. (1996, see their Figure 5) showed a comparison of optical and UV temperatures of DB white dwarfs using model atmospheres calculated with the ML1, ML2, and ML3 versions of the mixing-length theory; the authors only concluded then that ML2 and ML3 provided a more satisfactory correlation than ML1. The spectroscopic analysis of Beauchamp et al. (1999), however, relied on model atmospheres calculated with a convective efficiency of ML2 $/ \alpha=1.25$, although details of this particular choice were not provided in that study. The spectroscopic analysis of the DB stars in the SPY survey by Voss et al. (2007), on the other hand, relied on models calculated with ML2/ $\alpha=0.6$, i.e. the same convective efficiency as for DA models. As shown in Figure 2, the strength of the helium lines in DB stars are significantly affected by the assumed parameterization of the mixing-length theory used in the model atmosphere calculations, in particular between $T_{\text {eff }} \sim 18,000 \mathrm{~K}$ and $30,000 \mathrm{~K}$. It is thus of utmost importance to calibrate the convective efficiency in DB stars as accurately as possible.

The $\log g$ versus $T_{\text {eff }}$ distribution of all DB stars in our sample is displayed in Figure 14 for three versions of the mixing-length theory, namely ML2/ $\alpha=0.75,1.25$, and 1.75. As expected, only objects with $18,000 \mathrm{~K} \lesssim T_{\text {eff }} \lesssim 30,000 \mathrm{~K}$ are affected by this parameterization. As discussed at the end of Section 2.2, if the convective efficiency is underestimated, the helium lines are predicted too strong near the maximum equivalent widths, and DB stars will have a tendency to be lumped on either side of this maximum. This is precisely what is observed in Figure 14 for $\alpha=0.75$ where the maximum occurs near 23,000 K according to Figure 2 (see also Figure 3 of Bergeron et al. 1995 for DA stars near the ZZ Ceti instability strip). On the other hand, if the convective efficiency is overestimated, helium lines are predicted too weak near the maximum, and DB stars in this case will tend to accumulate at

\footnotetext{
${ }^{5}$ The more recent analysis by Tremblay \& Bergeron (2009), based on improved Stark profiles, suggests a more efficient value of $\alpha=0.8$.
} 
this location. Again, this is what is observed in Figure 14 for $\alpha=1.75$ where DB stars form a clump at $T_{\text {eff }} \sim 25,000 \mathrm{~K}$, even leaving a void near 20,000 K (see a similar result for DA stars in Bergeron et al. 1995). The results with $\alpha=1.25$, however, provide a smoother distribution and a uniform increase of the number of DB stars as a function of effective temperature, which is exactly what is expected in terms of the white dwarf luminosity function. Based on these results alone, a value around $\alpha=1.25$ seems entirely reasonable, even though we cannot easily rule out values between $\alpha \sim 1.0$ and 1.5 at this stage.

Bergeron et al. (1995) went a step further to calibrate the convective efficiency in DA stars, by comparing optical temperatures determined from spectroscopy with those obtained from UV energy distributions. Such a comparison is displayed in Figure 15 for two versions of the convective efficiency, namely ML2/ $\alpha=1.25$ and 1.75. Also shown in the bottom panel is a comparison of our UV temperatures, derived from fits to $I U E$ spectra with ML2/ $\alpha=0.6$ models, with those obtained by Castanheira et al. (2006) based on similar assumptions; note the almost perfect agreement here. In all UV fits, we simply assume $\log g=8$ and pure helium compositions for all objects. We first begin by examining the results for $\alpha=1.25$. Although there is a good overall agreement between optical and UV temperatures, there are also several discrepant results at the hot end of the sequence. The two objects with $T_{\text {opt }} \sim 30,000 \mathrm{~K}$ correspond to PG 0112+104, recently analyzed in great detail by Dufour et al. (2010a), and PG 1654+160. Although the IUE spectrum of the first object is of good quality, the spectrum of the second object suffers from a significant discontinuity between the SWP and LWP images. It is also possible that the UV energy distributions may become increasingly less sensitive to temperature at the hot of the DB sequence, while interstellar extinction may have to be taken into account for the intrinsically brighter, and more distant, DB stars. Indeed, for PG 0112+104, Dufour et al. (2010a) found the energy distribution extending into the FUV region $(\sim 925 \AA)$ globally consistent with the $\sim 31,000 \mathrm{~K}$ effective temperature derived from the optical spectroscopy provided that a small amount of reddening were included in the fits $(E(B-V)=0.015)$. The most important outlier in Figure 15 is LP 497-114 $(1311+129)$ with a $\sim 8000 \mathrm{~K}$ temperature difference; this peculiar white dwarf is further discussed in Section 5.4.

More worrisome in the top panel of Figure 15 is the systematic offset between both temperature scales (with $T_{\mathrm{UV}}>T_{\mathrm{opt}}$ ) for $15,000 \mathrm{~K}<T_{\mathrm{UV}}<22,000 \mathrm{~K}$. Note that a more efficient prescription of the mixing-length theory, shown in the middle panel of Figure 15, improves the agreement between both temperature scales near $T_{\mathrm{UV}} \sim 22,000 \mathrm{~K}$, but the agreement gets worse for hot DB stars, and the systematic shift still remains for $15,000 \mathrm{~K}$ $<T_{\mathrm{UV}}<20,000 \mathrm{~K}$. Hence, it is not clear that increasing the convective efficiency would improve the overall agreement. 
Since UV temperatures may depend on surface gravity, hydrogen abundance, and convective efficiency, we explore in Figure 16 the effects of variations of these parameters on our temperature determinations. All in all, we see that UV temperatures are fairly independent of our initial assumption of $\log g=8$ and pure helium compositions in our fits. The same conclusion applies to our particular choice of $\alpha$. We thus conclude that the systematic shifts observed in Figure 15 with our ML2/ $\alpha=1.25$ models cannot be explained in terms of inadequate assumptions in our fits to UV data. What the overall results suggest, instead, is that theoretical improvements probably need to be made at the level of the model atmospheres, perhaps even with the treatment of convective energy transport (see below).

\subsection{Error Estimation}

The internal uncertainties of the atmospheric parameters $-T_{\text {eff }}, \log g$, and $\mathrm{H} / \mathrm{He}-$ can be obtained directly from the covariance matrix of our fitting method. These depend mostly on the signal-to-noise ratio of the observations and on the sensitivity of the models to each parameter. For DA white dwarfs, these internal errors can become negligibly small with sufficiently high signal-to-noise spectroscopic observations $(\mathrm{S} / \mathrm{N} \gtrsim 50$; Bergeron et al. 1992, Liebert et al. 2005). Since our DB spectra are rather homogeneous in terms of S/N (see Figures 5 and 66) and of sufficiently high quality $(\mathrm{S} / \mathrm{N}>50)$, the internal errors in our analysis will be mostly dominated by the sensitivity of the models to each fitted parameter. The theoretical spectra displayed in Figure 1 indicate that the internal errors will be relatively small for $\log g$ (similar conclusions apply to $\mathrm{H} / \mathrm{He}$ ), but can be relatively large for $T_{\text {eff }}$, particularly in the temperature range where the strength of the helium lines reaches its maximum.

The true error budget, however, must also take into account external uncertainties originating mostly from flux calibration, which can be estimated from multiple observations of the same star, as performed by Liebert et al. (2005, Figure 8) for DA stars, or by Voss et al. (2007, Figures 1 and 2) for DB stars. Even though we have not reobserved DB stars for that specific purpose (except in a few cases), we still managed to secure repeated observations of 28 DB stars in our sample, either because of flux calibration problems (e.g., objects observed at high airmass), or insufficient $\mathrm{S} / \mathrm{N}$. Hence the external errors determined below will tend to be overestimated, if anything.

\footnotetext{
${ }^{6}$ In light of these results, we are surprised that Castanheira et al. (2006) succeeded in constraining $\log g$ by using only IUE data (see their Table 1); note that their $\log g$ determinations were omitted in the comparison displayed in Figure 4 of Voss et al. (2007).
} 
We compare in Figure 17 the $T_{\text {eff }}$ and $\log g$ measurements for the 28 DB stars in our sample with mutliple spectra. As expected, the external error on $T_{\text {eff }}$ are particularly large between $\sim 20,000 \mathrm{~K}$ and 25,000 K, where the helium lines reach their maximum strength. Otherwise, outside this temperature range, the temperature estimates are in excellent agreement. The spread in $\log g$ measurements is more significant, however, but some of the most extreme outliers are white dwarfs with a first spectroscopic observation at very low $\mathrm{S} / \mathrm{N}$; these include the DB stars reported by Lépine et al. (2011) for instance. If we take the average uncertainties of both parameters, we obtain $\left\langle\Delta T_{\text {eff }} / T_{\text {eff }}\right\rangle=4.6 \%$ and $\langle\Delta \log g\rangle=0.069$, but if we remove the obvious outliers, these numbers drop to $\left\langle\Delta T_{\text {eff }} / T_{\text {eff }}\right\rangle=2.3 \%$ and $\langle\Delta \log g\rangle=0.052$. Given that these are probably overestimated, as discussed in the previous paragraph, we adopt these values as conservative estimates of the external uncertainties of our atmospheric parameters. Note that these results are comparable to those obtained by Voss et al. (2007), $\left\langle\Delta T_{\text {eff }} / T_{\text {eff }}\right\rangle=2.03 \%$ and $\langle\Delta \log g\rangle=0.058$, based on multiple observations of 24 objects.

\subsection{Adopted Atmospheric Parameters}

The atmospheric parameters for the 108 DB and DBA stars in our sample are reported in Table 1 ; the values in parentheses for $T_{\text {eff }}$ and $\log g$ represent the combined (in quadrature) internal and external errors of the fitting technique, while only the internal error is available for $\mathrm{H} / \mathrm{He}$. For DB stars without detectable hydrogen, upper limits on the hydrogen abundance are given in Table 1 . The stellar mass $(M)$ and white dwarf cooling age $(\log \tau)$ of each star are obtained from evolutionary models similar to those described in Fontaine et al. (2001) but with $\mathrm{C} / \mathrm{O}$ cores, $q(\mathrm{He}) \equiv \log M_{\mathrm{He}} / M_{\star}=10^{-2}$ and $q(\mathrm{H})=10^{-10}$, which are representative of helium-atmosphere white dwarfs. The absolute visual magnitude $\left(M_{V}\right)$ and luminosity $(L)$ are determined with the improved calibration from Holberg \& Bergeron (2006), defined with the Hubble Space Telescope absolute flux scale of Vega. The $V$ magnitude 7 are taken from the Villanova White Dwarf Catalog, which combined with $M_{V}$ yield the distance $D$. Finally, for all PG stars in the complete sample, we also provide the $1 / V_{\max }$ weighting $\left(\mathrm{pc}^{-3}\right)$ used in the calculation of the luminosity function, where $V_{\max }$ represents the volume defined by the maximum distance at which a given object would still appear in the sample given the magnitude limit of the PG survey (see Liebert et al. 2005 for details).

Sample fits for both DB and DBA stars covering the full temperature range of our sample

\footnotetext{
${ }^{7}$ Note that these sometimes represent photographic magnitudes, Strömgren $y$ magnitudes, or even $B$ magnitudes.
} 
are displayed in Figure 18. The left panels show the blue portion of our spectroscopic fits, while the right panels show the corresponding region near $\mathrm{H} \alpha$. The importance of the red coverage is clearly illustrated, with $\mathrm{H} \alpha$ being barely detected in several DBA stars, while $\mathrm{H} \beta$ remains spectroscopically invisible. In other objects, only an upper limit on $\mathrm{H} / \mathrm{He}$ could be set, based on the absence of $\mathrm{H} \alpha$. We also show in this figure the DBA star with the largest hydrogen abundance measured in our sample (LP 497-114; 1311+129). A trend for the coolest objects to have larger than average surface gravities is already apparent.

\section{Selected Results}

\subsection{Comparison with Results from SPY}

As mentioned in the Introduction, the spectroscopic analysis of $\sim 70 \mathrm{DB}$ and DBA stars in the SPY survey (Voss et al. 2007) represents the largest set of data against which our atmospheric parameter determinations can be compared. We have 44 white dwarfs in common with the SPY sample, 22 of which are DBA stars. The comparison of effective temperatures and surface gravities is displayed in Figure 19.

Voss et al. (2007) also included van der Waals broadening in their models, in a simplified form. Even though they found a much better agreement with the observed line profiles and strengths, their fits were not completely satisfactory according to the authors; they also mention that a fit in which $\log g$ is actually allowed to vary converges to very high values. Hence a value of $\log g=8$ was simply assumed for the coolest DB stars in their sample (shown as open circles in Figure 19). The comparison of $\log g$ values is thus meaningless for these stars, although we note that the corresponding temperatures are in excellent agreement, except for the two coolest white dwarfs for which our values of $\log g \sim 9$ are likely overestimated. Worth mentioning in this context are the results shown in Figure 5 of Kepler et al. (2007) for the DB stars identified in the Data Release 4 of the SDSS, where the masses gradually increase to very large values $\left(M>1.0 M_{\odot}\right)$ at low effective temperatures. Whether this increase is due to an inappropriate treatment of van der Waals broadening, or to the neglect of this broadening mechanism altogether, is not discussed in their analysis.

For $T_{\text {eff }} \lesssim 19,000 \mathrm{~K}$, the $T_{\text {eff }}$ determinations displayed in Figure 19 are in good agreement, although our $\log g$ values in this temperature range appear systematically larger than those of Voss et al. by 0.15 dex, on average. The $\log g$ values agree much better at higher temperatures, however, with the exception of PG 1115+158 (labeled in the figure) for which we get a surface gravity 0.4 dex higher than the value derived by Voss et al. $(\log g=7.52)$. This white dwarf is of particular interest because of its low inferred mass of only $0.385 M_{\odot}$, 
and also because it is a member of the pulsating V777 Her class. Beauchamp et al. (1996) found no low-mass DB stars in their sample and argued that evolutionary scenarios that could produce such low-mass degenerates could simply not form DB white dwarfs. Both spectroscopic solutions for PG 1115+158 are compared in Figures 11 and 20. While the effective temperatures agree well within the uncertainties, the low surface gravity obtained by Voss et al. predicts helium lines that are much shallower than observed. Hence we believe that our solution at $\log g=7.91$ ( or $M=0.56 M_{\odot}$ ) is more appropriate for this star.

The differences in temperature for $T_{\text {eff }}>19,000 \mathrm{~K}$ are significantly more important, partly due to the use of different versions of the mixing-length theory (ML2/ $\alpha=0.6$ versus $\alpha=1.25$ ), but also because of the intrinsic difficulty of measuring $T_{\text {eff }}$ precisely in this particular temperature range. The most extreme case here is for KUV 03493+0131 (0349+015; labeled in Figure 19) for which we obtain an effective temperature $\sim 6000 \mathrm{~K}$ higher than Voss et al. Both spectroscopic solutions for this object are displayed in Figures 18 and 20. Note that if we rely only on the blue spectrum for this object, we find a cooler solution at $T_{\text {eff }}=22,760 \mathrm{~K}$, still significantly hotter than Voss et al. We find that our solution at $T_{\text {eff }}=24,860 \mathrm{~K}$ reproduces the widths and overall strengths of the neutral helium lines, as well of the observed slope of the spectral energy distribution (not shown here), much better than a solution at $18,740 \mathrm{~K}$.

\subsection{Mass Distribution}

Beauchamp et al. (1996) showed that the mass distribution of DB stars was relatively narrow, with the mass of $73 \%$ of their $41 \mathrm{DB}$ stars above $T_{\text {eff }}=15,000 \mathrm{~K}$ (excluding DBA stars) lying between 0.5 and $0.6 M_{\odot}$, for an average mass of $\left\langle M_{\mathrm{DB}}\right\rangle=0.567 M_{\odot}$. The 13 DBA stars were found at slightly larger masses, with an average mass of $\left\langle M_{\mathrm{DBA}}\right\rangle=0.642$ $M_{\odot}$, suggesting that more massive white dwarfs may have a tendency to show hydrogen, perhaps through an increased hydrogen accretion from the interstellar medium, or through a decreased dilution of the accreted hydrogen throughout a thinner helium convection zone (Beauchamp et al. 1996). One significant distinction between the mass distributions of DB and DA stars, noted by Beauchamp et al., was the almost complete absence of the lowand high-mass tails in the mass distribution of DB stars, suggesting that the formation of double degenerates and mergers, which are often invoked to explain respectively these low- and high-mass tails for DA stars, are not producing DB stars. The picture drawn by Voss et al. (2007) using $\sim 50$ DB stars from the SPY survey differs significantly from the one described above. While the results of Beauchamp et al. (1996) indicate that $25 \%$ of all DB white dwarfs are DBA stars, the high-resolution spectroscopic observations at $\mathrm{H} \alpha$ 
of the SPY survey revealed a much larger fraction of $55 \%$ of DBA stars in their sample, as discussed above. The resulting mean mass of each subsample, $\left\langle M_{\mathrm{DB}}\right\rangle=0.584 M_{\odot}$ and $\left\langle M_{\mathrm{DBA}}\right\rangle=0.607 M_{\odot}$, are now in much closer agreement, suggesting that both populations have a common origin. Furthermore, Voss et al. found a significantly larger mass dispersion, with several low-mass $\left(M \lesssim 0.5 M_{\odot}\right)$ and high-mass white dwarfs in their sample, in line with the results for DA stars.

The distribution of mass as a function of effective temperature for all 108 DB and DBA stars in our sample is displayed in Figure 21. Our final sample comprises 47 DBA stars, or $44 \%$ of the DB white dwarf population. Based on our discussion above, it is clear that this fraction represents only a lower limit since we are lacking the red spectral coverage for many of the DB stars in our sample, the cool ones in particular. In contrast to the mass distribution of DB stars in the SDSS obtained by Kepler et al. (2007), see their Figure 5), our mass distribution does not show this overwhelming increase in mass at low effective temperature, even though we do find several stars with masses in excess of $1 M_{\odot}$. These are (below $T_{\text {eff }}=15,000 \mathrm{~K}$ ) 0249+346, 1419+351, 1542-275, 2058+342, 2147+280, and 2316-273, all of which are shown in the last panel of Figure 5. Note that there are additional white dwarfs in the same range of temperature that have more normal masses $\left(M<0.8 M_{\odot}\right)$, for instance $0517+771,1056+345,1129+373,1542+244$, also shown in this last panel, with the exception that these have well defined helium absorption features - the $\log g$-sensitive He I $\lambda 3819$ line in particular — as opposed to the very massive ones. Instead of invoking a problem with the model spectra, our results suggest that we have probably reached the limit of the spectroscopic technique for these objects. They are probably cooler, more normal mass DB stars, with extremely weak absorption features (see further evidence below).

Even if we ignore the most massive objects in Figure 21, we still note a trend for DB stars in the range $13,000 \mathrm{~K} \lesssim T_{\text {eff }} \lesssim 18,000 \mathrm{~K}$ to have larger masses, up to $\sim 0.9 M_{\odot}$ in some cases, a problem usually attributed to the treatment of van der Waals broadening (Beauchamp et al. 1996). What is more intriguing, however, is that white dwarfs with normal masses, $M \sim 0.6 M_{\odot}$, are also found in the same range of temperature as these massive stars, which implies that the large dispersion in mass that occurs at these temperatures might be real after all. This point has already been made by Limoges \& Bergeron (2010) who show in their Figure 7 the spectroscopic fits for two DB stars with nearly identical temperatures $\left(T_{\text {eff }} \sim 15,000 \mathrm{~K}\right)$, but with $\log g$ values that differ by $0.33 \operatorname{dex}(\log g=8.03$ and 8.36$)$. The comparison reveals that the He I $\lambda 3819$ and $\lambda 4388$ lines, which are the most gravity sensitive in this range of temperature, differ markedly in both stars, indicating that the high $\log g$ value measured in one of these stars is probably real, and not a simple artifact produced by the models. 
The problem thus rests on our ability to confirm independently the atmospheric parameters obtained here within the current theoretical framework. To do so we rely on independent distance estimates obtained from trigonometric parallax measurements taken mostly from the Yale parallax catalog (van Altena et al. 1994, hereafter YPC) and from the new Hipparcos-based parallaxes from Gould \& Chanamé (2004). We found good trigonometric parallaxes for $11 \mathrm{DB}$ white dwarfs in our sample. The parallax and corresponding distance for each object are reported in Table 2 together with our atmospheric parameter solution, including the spectroscopic distance. The comparison of spectroscopic distances and those obtained from trigonometric parallaxes is displayed in Figure 22. The agreement is surprisingly good, especially given the fact that white dwarfs with the closest match are found in the temperature range where the mass dispersion is large in Figure 21 ( $T_{\text {eff }} \sim 15,000 \mathrm{~K}$; see Table 2). We are thus fairly confident that our surface gravity and mass values measured spectroscopically are fairly accurate.

We also observe some discrepant distance estimates, labeled in Figure 22, For Feige 4 $(0017+136$; label 1), we obtain a spectroscopic distance twice as large as that inferred from the trigonometric parallax. Our fit to this DB star is excellent, and we find no easy way to reconcile the two distance estimates. GD $358(1645+325$; label 2) is a pulsating white dwarf, the only hot DB star in Table 2. Again we do not have an easy explanation for the discrepancy. G188-27 (2147+280; label 3) has a spectroscopic distance significantly smaller than that inferred from the parallax. In this case, however, the spectroscopic $\log g$ value of 8.85 ( or $M=1.12 M_{\odot}$ ) is certainly overestimated, and this object corresponds to one of the (almost) featureless DB stars discussed earlier. A value of $\log g=8.2$ would actually reconcile both distance estimates perfectly. This corresponds to a mass of $\sim 0.7 M_{\odot}$, i.e. the average mass of the bulk of DB white dwarfs near 15,000 K.

The mass distribution of all DB and DBA stars in our sample, regardless of their temperature, is displayed in Figure 23. If we exclude from this distribution the most massive objects near $\sim 1.2 M_{\odot}$, which all correspond to the almost featureless cool DB stars discussed above, we find a mean mass for our sample of $\langle M\rangle=0.671 M_{\odot}$ with a standard deviation of only $\sigma_{M}=0.085 M_{\odot}$. In contrast to our preliminary conclusion presented in Beauchamp et al. (1996), we now find that there is no significant mass difference between the DB and the DBA samples $-\left\langle M_{\mathrm{DB}}\right\rangle=0.657 M_{\odot}$ and $\left\langle M_{\mathrm{DBA}}\right\rangle=0.688 M_{\odot}$ - in agreement with the conclusions of Voss et al. (2007). This result was already apparent from a quick examination of Figure 21. Note that the hottest white dwarfs in this figure are all of the DB type, and that their masses are slightly below $0.6 M_{\odot}$, thus contributing to an average mass for the DB white dwarfs that is only slightly lower than the mean for DBA stars.

We also compare, in Figure 23, the mass distribution of DB stars with that of DA stars 
from the PG survey, reanalyzed with our improved models for hydrogen-atmosphere white dwarfs (see Tremblay \& Bergeron 2011 and references therein), and for which we obtain $\left\langle M_{\mathrm{DA}}\right\rangle=0.631 M_{\odot}$ and $\sigma_{M}=0.133 M_{\odot}$. While the peak of the mass distribution of DB stars agrees with that of DA stars, the former distribution is shifted towards higher masses, resulting in an average mass $\sim 0.03 M_{\odot}$ higher. The peak value for the DB stars, between 0.60 and $0.65 M_{\odot}$, also agrees with the peak value determined by Voss et al. (2007, see their Figure 8), although their mean mass of $\langle M\rangle=0.596 M_{\odot}$ is much smaller than ours. This result is consistent with our $\log g$ determinations being larger than their values by about 0.15 dex in the temperature range where the bulk of DB white dwarfs is found (see Figure 19]).

Also of interest is the absence of low-mass DB stars in our sample, in contrast with low-mass DA stars, which are present in large numbers in all surveys, including the PG survey shown in Figure 23, Voss et al. (2007, see their Figure 8) identified three object.8 in the SPY sample with $M \lesssim 0.5 M_{\odot}$, the lowest mass DB white dwarf being PG $1115+158$, with a mass of only $0.385 M_{\odot}($ or $\log g=7.52)$. This object has already been discussed in Section 5.1, and we find instead a significantly larger value of $M=0.56 M_{\odot}$ for the same star. Similarly, Voss et al. report a mass of $M=0.481 M_{\odot}\left(T_{\text {eff }}=16,904 \mathrm{~K}\right)$ for HE 0423-1434, while we find $0.64 M_{\odot}\left(T_{\text {eff }}=16,900 \mathrm{~K}\right)$. Hence we reaffirm the conclusion of Beauchamp et al. (1996) that low-mass DB stars are rare, or absent, a conclusion also reached by Bergeron, Leggett, \& Ruiz (2001). These authors found, in their photometric analysis of 152 white dwarfs with trigonometric parallax measurements, that all low-mass degenerates probably possess hydrogen-rich atmospheres. As discussed in that study, since common envelope evolution is required to produce white dwarfs with $M \lesssim 0.5 M_{\odot}$ - the Galaxy being too young to have produced them from single star evolution — we must conclude that this particular evolutionary channel does not produce helium-rich atmosphere white dwarfs. This could be the case because the objects which go through this close-binary phase end up with hydrogen layers too massive to allow the DA to DB conversion near $T_{\text {eff }} \sim 30,000 \mathrm{~K}$, or below.

\subsection{Luminosity Function}

Liebert et al. (2005) presented spectrophotometric observations of a complete sample of 348 DA white dwarfs identified in the Palomar Green Survey and obtained robust values of $T_{\text {eff }}, \log g$, masses, radii, and cooling ages for all stars in their sample using the spectroscopic

\footnotetext{
${ }^{8}$ Note that we could only find two white dwarfs with $M<0.5 M_{\odot}$ in their Tables 1 and 2.
} 
technique. They also calculated the luminosity function of the sample, weighted by $1 / V_{\max }$, where $V_{\max }$ represents the volume defined by the maximum distance at which a given object would still appear in the sample given the magnitude limit of the PG survey. An overall formation rate of white dwarfs in the local Galactic disk of $1 \pm 0.25 \times 10^{-12} \mathrm{pc}^{-3} \mathrm{yr}^{-1}$ was also reported.

Since we observed all DB stars in the PG survey, we can calculate their luminosity function in the same fashion as for DA stars using the $1 / V_{\max }$ values given in Table 19 . Our results are reported in Table 3 and shown in Figure 24. Note that we rely here on bolometric magnitudes rather than absolute visual magnitudes — as Liebert et al. (2005) did (see their Figure 10) — since DA and DB stars have different $M_{V}$ values at a given effective temperature and surface gravity. Also shown in Figure 24 is the contribution from the DA stars in the PG survey, again reevaluated using our updated grid of DA model spectra. In the bolometric magnitude range over which DB stars are detected (roughly $\left.M_{\text {bol }}=7-11\right)$, the $\mathrm{DB} /(\mathrm{DA}+\mathrm{DB})$ ratio is of the order of 0.2 . We note, however, a large and significant increase in the value of $\log \phi$ in the range from $M_{\mathrm{bol}}=9$ to 10 , which corresponds to an effective temperature near 20,000 K. Thus, the DB/DA ratio is lower than the nominal value of 1 out of 4 white dwarfs at high effective temperatures, but increases sharply for stars below 20,000 K. This, it turns out, is also the temperature at which the bottom of the helium convection zone sinks sharply into the envelope along the cooling sequence (see Figure 3 ). It is also the temperature below which DBA stars start to appear in large numbers in Figure 21, and also where the average mass of all DB stars increases by $\sim 0.1 M_{\odot}$.

The total space density of DB stars in the PG survey can be obtained by summing the values in Table 3 over all magnitudes bins. We obtain a space density of $5.15 \times 10^{-5} \mathrm{DB}$ star per $\mathrm{pc}^{3}$. Hence we expect in a volume defined within $20 \mathrm{pc}$ from the Sun around two genuine DB stars, yet none have been identified in our sample (see also Holberg et al. 2008).

\subsection{Hydrogen Abundance and Mass Fraction in DB White Dwarfs}

About $44 \%$ of all DB stars in our sample show traces of hydrogen, or $50 \%$ if we consider only the objects for which we have spectroscopic data covering the $\mathrm{H} \alpha$ region. Voss et al. (2007) obtained an even higher fraction of $55 \%$ based on the SPY data. Hence the DBA phenomenon is quite common among DB stars. The hydrogen abundances as a function of effective temperature for all DBA stars in our sample are displayed in Figure 25. Also shown

\footnotetext{
${ }^{9}$ We ignore here the magnetic DB star PG $0853+163$ as well as the DB component of the double degenerate binary PG $1115+166$.
} 
are the upper limits on the hydrogen abundance for DB stars, as determined from the absence of $\mathrm{H} \alpha$ or $\mathrm{H} \beta$. In general, these DB stars are aligned on the observational limits reproduced here from Figure 12, but some objects have noisier data and these limits are simply not reached. The first striking result is that almost all white dwarfs above $T_{\text {eff }} \sim 20,000 \mathrm{~K}$ are DB stars, with the exception of 3 objects (KUV $05134+2605$, PG 1115+158, and PG $1456+103)$.

There are two possible origins for hydrogen in these helium-dominated atmospheres. First, hydrogen could be residual, originating from the thin hydrogen atmosphere of the DA progenitor, convectively diluted into the more massive helium envelope during the DA to DB transition that occurred at higher effective temperatures. MacDonald \& Vennes (1991) showed that the exact temperature at which this process occurs depends on the thickness of the hydrogen layer (see their Table 1). For their S1 models, which correspond to the Schwarzschild criterion with a value of $\alpha=1$, a DA star with hydrogen layer masses of $M_{\mathrm{H}}=10^{-15}, 10^{-14}$, and $10^{-13} M_{\odot}$ would respectively mix at temperatures of $T_{\text {eff }}=27,400 \mathrm{~K}$, $17,900 \mathrm{~K}$, and 11,700 K. The main effect of adding more hydrogen on top of the helium convection zone is to delay the time it takes for helium to develop a sufficiently deep convection zone, thus reducing the temperature at which mixing occurs. Upon mixing, it is then assumed that the thin hydrogen atmosphere will be thoroughly mixed with the more massive helium convection zone, and that the resulting photospheric hydrogen abundance will simply correspond to the ratio of the total hydrogen mass $M_{\mathrm{H}}$ to that of the helium convection zone, $M_{\mathrm{He}-\mathrm{conv}}$. One problem immediately arises when trying to account for the hot DB stars above $T_{\text {eff }} \sim 20,000 \mathrm{~K}$ in Figure 25. Indeed, for convective mixing to occur at these temperatures, the required hydrogen layer mass has to be of the order of $10^{-15} M_{\odot}$ according to MacDonald \& Vennes. This mass, it turns out, is comparable to the mass of the helium convection zone in this temperature range (see Figure 31). The complete dilution of hydrogen should thus lead to a $\mathrm{H} / \mathrm{He}$ ratio $\sim 1$ instead of the observed limits of $\mathrm{H} / \mathrm{He} \sim 10^{-4}-10^{-5}$.

We can explore this problem more quantitatively by converting the hydrogen abundances from Figure 25 into total hydrogen masses by assuming that hydrogen is indeed homogeneously mixed in the helium convection zone (see also Dufour et al. 2007a and Voss et al. 2007). For simplicity, we assume evolutionary models at $0.6 M_{\odot}$ (as in Figure 3) and we also ignore the feedback effect of the presence of hydrogen on the depth of the mixed $\mathrm{H} / \mathrm{He}$ convection zone10. The total hydrogen mass as a function of effective temperature for all DBA stars in our sample is displayed in Figure 26. Again, the values for DB stars represent only

\footnotetext{
${ }^{10}$ The values of $M_{\mathrm{H}}$ inferred here differ somewhat from the values obtained by Dufour et al. (2007a, see Figure 13) since in their calculations, the depth of the helium convection zone was taken from Table 1 of Dupuis et al. (1993), while we rely here on the more recent evolutionary models of Fontaine et al. (2001).
} 
upper limits. Above $T_{\text {eff }}=22,000 \mathrm{~K}$, these upper limits imply $M_{\mathrm{H}} \lesssim 10^{-17} M_{\odot}$. Such small hydrogen layer masses are simply unable to maintain a hydrogen-rich atmosphere at higher temperatures. The only way to account for the existence of these hot DB stars in our sample is thus to conclude that they must have maintained a helium-dominated atmosphere through their entire life history. This interpretation is consistent with the existence of hot DB stars in the DB gap, i.e. $30,000 \mathrm{~K} \lesssim T_{\text {eff }} \lesssim 45,000 \mathrm{~K}$ (Eisenstein et al. 2006). Even the three DBA stars near 24,000 $\mathrm{K}$ have hydrogen layer masses $\left(M_{\mathrm{H}} \sim 10^{-16} M_{\odot}\right)$ too small to have been DA stars in the past according to the results shown Figure 1 of MacDonald \& Vennes (1991). However, our estimates are sufficiently approximate that larger masses of the order of $M_{\mathrm{H}} \sim 10^{-15} M_{\odot}$ can probably be accommodated in these objects, in which case they would have undergone the DA to DB transition near $\sim 27,000 \mathrm{~K}$ according to Table 1 of MacDonald \& Vennes (1991).

We note that a similar evolutionary channel is required to explain the existence of the Hot DQ stars, whose atmospheres are dominated by carbon, with only traces of helium and no hydrogen (Dufour et al. 2008). Since the hottest DQ stars known have temperatures around 24,000 K, their immediate progenitors must necessarily be DB stars with no hydrogen. Hence it is tantalizing to suggest that some of the hot DB stars observed here, as well as the hot DB stars in the gap identified in the SDSS, are the immediate progenitors of the Hot DQ stars. If this interpretation is correct, what fraction of DB stars between $T_{\text {eff }} \sim 30,000 \mathrm{~K}$ and 24,000 K are progenitors of Hot DQ stars remains to be determined.

The situation is even more complicated below $T_{\text {eff }} \sim 20,000 \mathrm{~K}$ where the inferred hydrogen layer masses in the DBA stars are in the range $10^{-12} \lesssim M_{\mathrm{H}} / M_{\odot} \lesssim 10^{-10}$. According to MacDonald \& Vennes (1991), DA stars with such large amounts of hydrogen would only mix at temperatures below 12,000 K. In other words, we observe much more hydrogen in those stars than would be expected on the basis of the complete mixing of the hydrogen layer in the underlying helium convection zone. Hence, the hydrogen abundances in these DBA stars are too high to have a residual origin, and external sources of hydrogen must be invoked, either from the interstellar medium or from other bodies such as comets, disrupted asteroids, small planets, etc., a conclusion also reached by Voss et al. (2007), see also MacDonald \& Vennes 1991). It is important to recall that hydrogen can only accumulate in the photospheric regions, as a result of accretion, while heavier elements will slowly diffuse at the bottom of the helium convection zone on time scales of the order of $10^{5}$ years or less (see Table 1 of Dupuis et al. 1993). Since the bottom of the helium convection zone grows deeper with time (see Figure 3), hydrogen becomes diluted in a larger volume. The measured hydrogen abundances in cool DB stars, and eventually DC white dwarfs, are thus governed by this balance between accretion and dilution within the helium convection zone. It is thus important to connect the constraints imposed by DBA stars with independent determina- 
tions of the hydrogen abundances in cooler white dwarfs, such as DZA stars (Dufour et al. 2007a).

We show in Figure27 the same results as in Figure 25, but we added the $\mathrm{H} / \mathrm{He}$ abundance ratios predicted from continuous accretion from the interstellar medium at various rates, from $10^{-21}$ to $10^{-17} M_{\odot} \mathrm{yr}^{-1}$ (see also Figure 10 of Voss et al. 2007 for a similar calculation). Also shown are the hydrogen abundances for the DZA stars determined by Dufour et al. (2007a). As can be seen, with the exception of the three hottest DBA stars, this range of accretion rates can easily account for the amount of hydrogen observed in DBA and DZA stars. However, some of the upper limits on the hydrogen abundance below $\sim 20,000 \mathrm{~K}$ are quite stringent, $\mathrm{H} / \mathrm{He}<10^{-6}$. These DB stars are observed in the same range as some DBA stars, characterized by hydrogen abundances of the order of $\mathrm{H} / \mathrm{He} \sim 10^{-4}$, or a factor 100 higher. It is thus difficult to understand why accretion of hydrogen could be effective for some DBA stars, and not for other white dwarfs at the same temperature. The existence of such cool, hydrogen-deficient DB stars in our sample, and probably a significant fraction of cool DC and DZ stars as well, can only be interpreted as having evolved from hotter DB stars that contain negligible amounts of hydrogen in their atmospheres, without invoking any accretion mechanism whatsoever.

If this interpretation is correct, the presence of hydrogen in DBA stars must be residual. Perhaps then, it is time to question the assumption of complete mixing of the hydrogen layer: in that case, it remains conceivable that the amount of hydrogen present is indeed of the order of $\sim 10^{-14} M_{\odot}$, i.e. the amount expected for the convective dilution of the hydrogen atmosphere to occur below $T_{\text {eff }} \sim 20,000 \mathrm{~K}$ according to MacDonald \& Vennes (1991), but that it somehow floats on top of the photosphere rather than being forcefully mixed by the helium convection zone.

One particular object in our sample is LP 497-114 (1311+129), the DBA star with the largest hydrogen abundance, $\mathrm{H} / \mathrm{He} \sim 10^{-3}$ (at $T_{\text {eff }} \sim 19,000 \mathrm{~K}$ in Figure 25). We have three independent spectroscopic observations for this object, one of which is a SDSS spectrum (which serves also as our $\mathrm{H} \alpha$ spectrum). Our best fit to these spectra are displayed in Figure 28, The two bottom fits are clearly at odds with what we can usually achieve in this temperature range, especially given the high signal-to-noise ratio of these observations. In particular, the region around He I $\lambda 4471$, as well as the core of most neutral helium lines, are poorly reproduced. If we consider only the blue spectra, we can achieve much better fits at $T_{\text {eff }} \sim 23,000 \mathrm{~K}$, but then the line cores of $\mathrm{H} \alpha$ and He I $\lambda 6678$ are predicted much too shallow. Note that the fit to the SDSS spectrum (top) is less problematic, although the line cores are also predicted too shallow. Incidentally, LP 497-114 shows the worst agreement between optical and UV temperatures $\left(T_{\mathrm{UV}} \sim 27,000 \mathrm{~K}\right.$ in Figure 15). Perhaps the problem 
with the modeling of this star is the assumption of a homogeneously mixed hydrogen and helium atmospheric composition. This star might be in the process of being convectively mixed, with hydrogen constantly trying to float back to the surface. This could even explain the small spectroscopic variations from spectrum to spectrum observed in Figure 28. If this is the case, the chemically homogeneous models used here no longer apply. This might also be the case, but to a lesser extent, with other DBA stars in our sample.

\subsection{The Instability Strip of the Pulsating V777 Her Stars}

Our first assessment of the instability strip of the pulsating DB (V777 Her) stars was presented in the spectroscopic study of Beauchamp et al. (1999). The picture at that time was complicated by the fact that only blue spectroscopic observations were available, and the insufficient constraints on the hydrogen content is these stars did not allow us to choose the most appropriate solution. Hunter et al. (2001) presented an update of the instability strip of the V777 Her stars using new spectroscopic observations at $\mathrm{H} \alpha$, with the specific aim of constraining the hydrogen abundance in these stars. The results, shown in Figure 2 of Hunter et al., reveal an instability strip that contains at least one non-variable white dwarf, as well as a significant number of unknowns. Since this last report, one object in this sample, PG 2246+121, has been identified as a new variable DB white dwarf by Handler (2001).

We show in Figure 29 the results from our revised spectroscopic analysis. The V777 Her stars are identified in Table 1. We distinguish in this plot variable and non-variable white dwarfs, but also DB and DBA stars. Above $T_{\text {eff }} \sim 20,000 \mathrm{~K}$, there are only 3 DBA stars in our sample with hydrogen abundances around $\mathrm{H} / \mathrm{He} \sim 10^{-3.5}$, i.e. about one dex larger than the upper limits derived for DB stars in the same range of temperature, and all three are pulsating white dwarfs. Given our results presented so far, DB and DBA stars in this range of effective temperature seem to form two distinct classes of objects, with probably different progenitors: hot DB stars that once resided in the gap in one case, DA stars with thin hydrogen layers $\left(M_{\mathrm{H}} \sim 10^{-15} M_{\odot}\right)$ in the other case. Our results displayed in Figure 29 are consistent with this idea, in the sense that we can easily identify two distinct pure instability strips, within the uncertainties, one for the pure helium-atmosphere DB stars, and one for the DBA stars.

Also displayed in Figure 29 are the theoretical blue edges for pure helium envelope models reproduced from Figure 3 of Beauchamp et al. (1999), based on the nonadiabatic calculations of Fontaine \& Brassard (1997) and Brassard \& Fontaine (1997), for various versions of the mixing-length theory. For DB stars, the observed blue edge near $T_{\text {eff }} \sim 30,000 \mathrm{~K}$ suggests 
a convective efficiency somewhere between the ML2 $(\alpha=1.0)$ and ML3 ( $\equiv$ ML2/ $\alpha=2.0)$ versions of the mixing-length theory, a parameterization entirely consistent with that adopted in our model atmosphere calculations (ML2/ $\alpha=1.25)$. The location of the red edge of the DB instability strip is located near $T_{\text {eff }} \sim 25,000 \mathrm{~K}$, for a corresponding strip about $5000 \mathrm{~K}$ wide. Two objects, shown as black open circles, are located at the red edge - KUV $03493+0131(0349+015)$ and PG $2354+159$ - but only the latter has been confirmed as a non-variable, to our knowledge (Robinson \& Winget 1983).

The boundaries of the instability strip for the DBA stars are not as well defined as for the DB white dwarfs since we lack non-variable DBA stars both hotter and cooler than the DBA pulsators studied here, which would allow us to define empirically the edges of the instability strip (for instance, there is no DBA star in our sample between $T_{\text {eff }} \sim 23,000 \mathrm{~K}$ and 20,000 K). Nevertheless, the existence of DBA pulsators cooler than the coolest variable DB stars suggests that the presence of small traces of hydrogen $\left(\mathrm{H} / \mathrm{He} \sim 10^{-3}\right)$ in the partial ionization zone would lower the temperature at which helium-atmosphere white dwarfs can pulsate. Interestingly, the effect produced by the presence of small amounts of hydrogen on the theoretical blue edge of the instability strip has been presented in Figure 10 of Fontaine \& Brassard (2008). Indeed, the blue edge becomes cooler, but the effect is rather small $(\sim 500 \mathrm{~K})$, although the authors considered a trace of only $\mathrm{H} / \mathrm{He}=10^{-4}$ in their calculations, and it is expected that the effect would be considerably more important with the higher hydrogen abundances inferred in our analysis.

What finally comes out of these results is that DB white dwarfs cannot be considered as a homogeneous class of objects when studying the location of the instability strip because of this additional parameter, the hydrogen abundance.

\subsection{The Case of HE 2149-0516}

HE 2149-0516 is a DAB star discussed briefly by Voss et al. (2007), who describe its spectrum as exhibiting strong Balmer lines and weaker but strong neutral helium lines. The authors had difficulty fitting this object with either pure hydrogen or pure helium models, and only a pure hydrogen fit to $\mathrm{H} \alpha$ suggested a temperature near $T_{\text {eff }} \sim 30,000 \mathrm{~K}$, with an extremely low surface gravity of $\log g \sim 7$. Our normalized spectrum for this peculiar white dwarf is displayed in Figure 30. The absolute energy distribution (not shown here) is almost flat in the wavelength range displayed here, so this cannot be a hot white dwarf. Our best solution with our grid of DB models indeed yields $T_{\text {eff }}=11,370 \mathrm{~K}, \log g=7.6$, and $\log$ $\mathrm{H} / \mathrm{He}=-4.5$, but our spectroscopic fit completely fails to reproduce both the helium and

hydrogen lines adequately. Instead, we could achieve a much better fit by assuming that HE 
2149-0516 is an unresolved DA + DB (or perhaps DBA) composite system. In this case, each component of the system dilutes the absorption features of the other component. Given that there are simply too many free parameters to fit and that the observed features are relatively weak, we kept both values of $\log g$ fixed in our fitting procedure, as well as the value of $\mathrm{H} / \mathrm{He}$ for the DB star, and experimented with various values of these parameters. Our best fit is displayed in Figure 30, This is admittedly not a perfect fit, especially in the blue region of the spectrum, but given that both the DA and DB components fall in a temperature range which is problematic in terms of the modeling (i.e., the high-log $g$ problem), we are rather satisfied with the quality of our fit. Note that the combined model fluxes also reproduce the slope of the observed energy distribution perfectly (not shown here).

\section{Conclusion}

We presented a comprehensive analysis of 108 DB white dwarfs using model atmosphere fits to blue and red spectroscopic observations. As in previous investigations, we showed that high signal-to-noise spectroscopic data near $\mathrm{H} \alpha$ are crucial to determine, or constrain, the hydrogen abundance in these white dwarfs. For the hottest stars in particular, such data allowed us to pinpoint more accurately the effective temperatures and surface gravities. While we demonstrated that these parameters depend on the assumed convective efficiency used in our model atmospheres, we also showed that a detailed parameterization of the

mixing-length theory is more difficult to achieve than in DA stars, partly because of the lack of sensitivity of the UV energy distributions to this parameter.

The mean mass of DB white dwarfs appears to be significantly larger than that of DA stars, especially at the cool end of the DB sequence. Since this corresponds to a temperature regime where the physics of line broadening becomes more questionable, van der Waals broadening in particular, we used trigonometric parallax measurements to demonstrate that the spectroscopic distances were in excellent agreement with those obtained from parallaxes, giving us confidence in our ability to model the DB stars with sufficient accuracy. We found no significant differences between the mass of DB and DBA stars, however, suggesting that both populations may have a common origin. DBA stars, on the other hand, are usually found at much lower temperatures than DB stars. While the luminosity functions of DA and DB stars identified in the PG survey indicate that $20 \%$ of all white dwarfs are DB stars, this appears to be true only below $T_{\text {eff }} \sim 17,000 \mathrm{~K}$ (i.e. $M_{\text {bol }}>9.5$ in Figure 24). At higher temperatures, only $\sim 9 \%$ of all white dwarfs are DB stars. This clearly indicates that the sudden increase in the ratio of DB to DA white dwarfs is the result of the transformation of DA stars into DB stars, but at a much lower temperature than the canonical value defined by 
the red edge of the DB gap — or DB deficiency — near 30,000 K, a conclusion also reached by Eisenstein et al. (2006).

The global picture that ultimately emerges from our study is that there are at least two evolutionary channels feeding the DB white dwarf population. A small fraction of white dwarf stars are born as hydrogen-deficient stars, with negligible amounts of hydrogen in their envelope. These will remain hydrogen-deficient throughout their subsequent evolution, and will never become DA stars. The existence of extremely hot DB stars in the DB gap certainly supports this conclusion. We suggest that the hottest DB stars in our sample with $T_{\text {eff }}>20,000 \mathrm{~K}$ descend directly from this evolutionary channel. In the bin centered on $M_{\text {bol }}=7.0$ in Figure 24, we actually find only 3 DB stars, which represent $5.6 \%$ of the entire white dwarf population in this particular bin. If we apply the same fraction to the brighter bin centered on $M_{\text {bol }}=6.0\left(T_{\text {eff }} \sim 35,000 \mathrm{~K}\right)$, which contains 24 DA stars, we should only expect a single DB white dwarf in this particular bin. It is then perhaps not surprising that none were found in the PG survey. Note that a hot DB star in the gap was actually identified in the KUV survey, but this star was hidden in an unresolved DA + DB double degenerate system (Limoges et al. 2009).

The hydrogen-deficient evolutionary channel is also supported by observations at low effective temperatures $\left(T_{\text {eff }} \sim 15,000 \mathrm{~K}\right)$ where we see a significant number of DB stars with extremely low hydrogen abundances $\left(\mathrm{H} / \mathrm{He}<10^{-6}\right)$. The mere existence of these almost pure helium atmospheres rules out the accretion from the interstellar medium scenario as the most likely source of hydrogen in the DBA stars found in the same temperature range. The only alternative, which is the second evolutionary channel, is that hydrogen must have a primordial origin, resulting from the transformation of a thin hydrogen-atmosphere DA white dwarf into a DB degenerate. The observed hydrogen abundances in DBA stars are too large, however, to be explained by a simple model where hydrogen is thoroughly mixed within the helium convection zone. Instead, we proposed a model where hydrogen tends to float on top of the photosphere rather than being completely mixed in the helium convective envelope. Since DBA stars appear in large numbers only below $T_{\text {eff }} \sim 20,000 \mathrm{~K}$, this suggests hydrogen layer masses of $M_{\mathrm{H}} \sim 10^{-14} M_{\odot}$ for most DA progenitors, although the existence of a few hotter DBA stars may indicate even thinner hydrogen layers in some cases.

In principle, Hot DQ stars must also be affecting the luminosity function of DB stars, either when DB stars are transformed into Hot DQ stars near $T_{\text {eff }} \sim 24,000 \mathrm{~K}$ (i.e., the hottest DQ stars currently known), most likely through convective mixing, or when Hot DQ stars apparently return to being DB stars near $T_{\text {eff }} \sim 18,000 \mathrm{~K}$ (i.e. the coolest DQ stars currently known), through a process currently unknown. But the number of known Hot DQ stars in the SDSS sample is so small, only 14 known to date, that the contribution of these 
stars to the luminosity function of DB stars is most certainly negligible.

We may speculate as to the origin of a larger mean mass for DB stars compared to DA stars. First, this may be an artifact associated with the model atmospheres. Indeed, the increase in mass becomes particularly important when the atmospheres of DB stars become strongly convective (see Figures 3 and 21). Perhaps this increase in mass is analog to that observed in cool $\left(T_{\text {eff }} \lesssim 12,000 \mathrm{~K}\right)$ DA stars, where the most likely explanation for this phenomenon has been attributed to the limitations of the mixing-length theory used in the model atmosphere calculations (Koester et al. 2009; Tremblay et al. 2010). It is possible that such limitations also apply to DB atmospheres. If the mean mass of DB stars is indeed significantly larger than that of DA stars, this implies that their progenitors on the main sequence must have been more massive as well. According to Werner \& Herwig (2006), the mechanism by which hydrogen is destroyed - during the post-asymptotic giant branch (AGB) phase - to the level required to account for the almost pure helium-atmosphere DB stars discussed here, or even the DBA stars in our sample, is probably caused by a very late helium-shell flash, or an AGB final thermal pulse. It is not inconceivable that such a mechanism is more efficient in more massive stars on the main sequence.

Our next task will be to study the numerous DB white dwarfs discovered in the Sloan Digital Sky Survey, even though the average signal-to-noise ratio of the spectroscopic observations is admittedly much lower than that of our sample (see Figure 6). Future work should also include a proper treatment of evolutionary models with mixed hydrogen and helium abundances to evaluate better the total hydrogen content of DBA stars.

The work reported here was supported in part by the NSERC Canada and by the Fund FQRNT (Québec). MTR received support from FONDAP Center for Astrophysics and PFB06 (CATA). PB is a Cottrell Scholar of the Research Corporation for Science Advancement, while $\mathrm{PaD}$ is a CRAQ postdoctoral fellow. We are grateful to the Steward Observatory, to the Kitt Peak National Observatory, and to the Carnegie Observatories for providing observing time for this project. 


\section{REFERENCES}

Achilleos, N., Wickramasinghe, D. T., Liebert, J., Saffer, R. A., \& Grauer, A. D. 1992, ApJ, 396,273

Beauchamp, A. 1995, Ph. D. thesis, Université de Montréal

Beauchamp, A. 1998, JRASC, 92, 126

Beauchamp, A., \& Wesemael, F. 1998, ApJ, 496, 395

Beauchamp, A., Wesemael, F., \& Bergeron, P. 1997, ApJ, 108, 559

Beauchamp, A., Wesemael, F., Bergeron, P., Fontaine, G., Saffer, R. A., Liebert, J., \& Brassard, P. 1999, ApJ, 516, 887

Beauchamp, A., Wesemael, F., Bergeron, P., \& Liebert, J. 1995, ApJ, 441, L85

Beauchamp, A., Wesemael, F., Bergeron, P., Liebert, J., \& Saffer, R. A. 1996, in HydrogenDeficient Stars, edited by C.S. Jeffery \& U. Heber, ASP Conference Series 96, San Francisco, 295

Bergeron, P., Leggett, S. K., \& Ruiz, M. T. 2001, ApJS, 133, 413

Bergeron, P., \& Liebert, J. 2002, ApJ, 566, 1091

Bergeron, P., Saffer, R. A., \& Liebert, J. 1992, ApJ, 394, 228

Bergeron, P., Wesemael, F., Lamontagne, R., Fontaine, G., Saffer, R. A., \& Allard, N. F. 1995, ApJ, 449, 258

Böhm, K.-H., \& Cassinelli, J. P. 1971, A\&A, 12, 21

Brassard, P., \& Fontaine, G. 1997, in Proc. 3rd Conf. on Faint Blue Stars, eds A. G. Davis Philip, J. Liebert, \& R. A. Saffer (Schenectady NY: L. Davis Press), 485

Castanheira, B. G., Kepler, S. O., Handler, G., \& Koester, D. 2006, A\&A, 450, 331

Dahn, C. C., et al. 1988, AJ, 95, 237

Deridder, G., \& Van Rensbergen, W. 1976, A\&AS, 23, 147

Desharnais, S., Wesemael, F., Chayer, P., Kruk, J. W., \& Saffer, R. A. 2008, ApJ, 672, 540

Dufour, P., Bergeron, P., Liebert, J., Harris, H. C., Knapp, G. R., Anderson, S. F., Hall, P. B., Strauss, M. A., Collinge, M. J., \& Edwards, M. C. 2007a, ApJ, 663, 1291 
Dufour, P., Desharnais, S., Wesemael, F., Chayer, P., Lanz, T., Bergeron, P., Fontaine, G., Beauchamp, A., Saffer, R. A., Kruk, J. W., \& Limoges, M.-M. 2010a, ApJ, 718, 647

Dufour, P., Fontaine, G, Liebert, J., Schmidt, G. D., \& Behara, N. 2008, ApJ, 683, 978

Dufour, P., Kilic, M., Fontaine, G., Bergeron, P., Lachapelle, F.-R., Kleinman, S. J., \& Leggett, S. K. 2010b, ApJ, 719, 803

Dufour, P., Liebert, J., Fontaine, G, \& Behara, N. 2007b, Nature, 450, 522

Dupuis, J., Fontaine, G., Pelletier, C., \& Wesemael, F. 1993, ApJS, 84, 73

Eisenstein D. J. et al. 2006, AJ, 132, 676

Farihi, J., Barstow, M. A., Redfield, S., Dufour, P., \& Hambly, N. C. 2010, MNRAS, 404, 2123

Fontaine, G., \& Brassard, P. 1997, in White Dwarfs: Proc. 10th European Workshop on White Dwarfs, ed. J. Isern, M. Hernanz, \& E. Garcia-Berro (Dordretch: Kluwer), 451

Fontaine, G., \& Brassard, P. 2008, PASP, 120, 1043

Fontaine, G., Brassard, P., \& Bergeron, P. 2001, PASP, 113, 409

Fontaine, G., \& Wesemael, F. 1987, in IAU Colloquium 95, The Second Conference on Faint Blue Stars, eds. A. G. Davis Philip, D. S. Hayes, \& J. Liebert (Schenectady: L. Davis), 319

Friedrich, S., Koester, D., Christlieb, N., Reimers, D., \& Wisotzki L. 2000, A\&A, 363, 1040

Gould, A., \& Chanamé, J. 2004, ApJS, 150, 455

Green, R. F., Schmidt, M., \& Liebert, J. 1986, ApJS, 61, 305

Handler, G. 2001, MNRAS, 323, L43

Holberg, J. B., Barstow, M. A., \& Burleigh, M. R. 2003, ApJS, 147, 145

Holberg, J. B., \& Bergeron, P. 2006, ApJ, 132, 1221

Holberg, J. B., Sion, E. M., Oswalt, T., McCook, G. P., Foran, S., \& Subasavage, J. P. 2008, AJ, 135,1225

Hummer, D. G., \& Mihalas, D. 1988, ApJ, 331, 794 
Hunter, C., Wesemael, F., Saffer, R. A., Bergeron, P., \& Beauchamp, A. 2001, in 12 ${ }^{\text {th }}$ European Conference on White Dwarfs, edited by J. L. Provencal, H. L. Shipman, J. MacDonald, \& S. Goodchild, ASP Conference Series 226, San Francisco, 153

John, T. L. 1994, MNRAS, 269, 871

Kepler, S. O., Kleinman, S. J., Nitta, A., Koester, D., Castanheira, B. G., Giovannini, O., Costa, A. F. M., \& Althaus, L. 2007, MNRAS, 375, 1315

Koester, D., Kepler, S. O., Kleinman, S. J., \& Nitta, A. 2009, Journal of Physics Conference Series, 172, 012006

Lépine, S., Bergeron, P., \& Lanning, H. H. 2011, AJ, 141, 96

Liebert, J., Bergeron, P., \& Holberg, J. B. 2005, ApJS, 156, 47

Limoges, M.-M., \& Bergeron, P. 2010, ApJ, 714, 1037

Limoges, M.-M., Bergeron, P., \& Dufour, P. 2009, ApJ, 696, 1461

MacDonald, J., \& Vennes, S. 1991, ApJ, 371, 719

Massa, D., \& Fitzpatrick, E. L. 2000, ApJS, 126, 517

Petitclerc, N., Wesemael, F., Kruk, F., Chayer, P., \& Billères, M. 2005, ApJ, 624, 317

Press, W. H., Flannery, B. P., Teukolsky, S. A., \& Vetterling, W. T. 1986, Numerical Recipes (Cambridge: Cambridge University Press)

Robinson, E. L. \& Winget, D. E. 1983, PASP, 95, 386

Tassoul, M., Fontaine, G., \& Winget, D.E. 1990, ApJS, 72, 335

Tremblay, P.-E., \& Bergeron, P. 2009, ApJ, 696, 1755

Tremblay, P.-E., Bergeron, P., \& Gianninas, A. 2011, ApJ, 730, 128

Tremblay, P.-E., Bergeron, P., Kalirai, J. S., \& Gianninas, A. 2010, ApJ, 712, 1345

van Altena, W. F., Lee, J. T., \& Hoffleit, E. D. 1994, The General Catalogue of Trigonometric Parallaxes (New Haven: Yale University Observatory) (YPC)

Voss, B., Koester, D., Napiwotzki, R., Christlieb, N., \& Reimers, D. 2007, A\&A, 470, 1079

Werner, K., \& Herwig, F. 2006, PASP, 118, 183 
Wesemael, F., Liebert, J., Schmidt, G. D., Beauchamp, A., Bergeron, P., \& Fontaine, G. 2001, ApJ, 554, 1118

Wickramasinghe, D. T. 1979, in White Dwarfs and Variable Degenerate Stars, edited by H. M. Van Horn \& V. Weidemann, University of Rochester, Rochester, 35 
Table 1. Atmospheric Parameters of DB and DBA Stars

\begin{tabular}{|c|c|c|c|c|c|c|c|c|c|c|c|c|}
\hline WD & Name & $T_{\text {eff }}(\mathrm{K})$ & $\log g$ & $\log \mathrm{H} / \mathrm{He}$ & $M / M_{\odot}$ & $M_{V}$ & $\log L / L_{\odot}$ & $V$ & $D(\mathrm{pc})$ & $1 / V_{\max }$ & $\log \tau$ & Notes \\
\hline $0000-170$ & G266-32 & $13,880(362)$ & $8.63(0.13)$ & $-5.64(0.48)$ & $0.99(0.08)$ & 12.49 & -2.67 & 14.69 & 27 & & 8.84 & \\
\hline $0002+729$ & GD 408 & $14,410(353)$ & $8.26(0.10)$ & $-5.97(0.82)$ & $0.75(0.07)$ & 11.79 & -2.36 & 14.33 & 32 & & 8.54 & \\
\hline $0017+136$ & Feige 4 & $18,130(440)$ & $8.09(0.07)$ & $-4.61(0.20)$ & $0.65(0.04)$ & 10.97 & -1.85 & 15.37 & 75 & $7.68(-7)$ & 8.12 & \\
\hline $0031-186$ & KUV 00312-1837 & $15,020(397)$ & $8.43(0.12)$ & $-5.36(0.34)$ & $0.86(0.08)$ & 11.96 & -2.39 & 16.66 & 87 & & 8.61 & \\
\hline $0100-068$ & G270-124 & $19,800(532)$ & $8.07(0.07)$ & $-5.08(0.93)$ & $0.64(0.04)$ & 10.78 & -1.68 & 13.95 & 43 & & 7.96 & \\
\hline $0112+104$ & PG $0112+104$ & $31,040(1058)$ & $7.83(0.06)$ & $<-3.84(0.84)$ & $0.53(0.03)$ & 9.89 & -0.74 & 15.36 & 124 & $1.80(-7)$ & 7.17 & \\
\hline $0125-236$ & G274-39 & $16,610(457)$ & $8.26(0.09)$ & $-5.31(0.38)$ & $0.75(0.06)$ & 11.44 & -2.11 & 15.38 & 61 & & 8.36 & \\
\hline $0129+246$ & PG $0129+247$ & $16,440(463)$ & $8.27(0.10)$ & $-5.26(0.40)$ & $0.76(0.07)$ & 11.48 & -2.13 & 16.09 & 83 & $1.49(-6)$ & 8.38 & \\
\hline $0211+646$ & Lan 150 & $20,060(596)$ & $8.01(0.07)$ & $<-3.91(0.18)$ & $0.60(0.04)$ & 10.66 & -1.62 & 17.43 & 226 & & 7.88 & \\
\hline $0214+699$ & Lan 158 & $29,130(1331)$ & $7.88(0.07)$ & $<-4.01(0.97)$ & $0.55(0.04)$ & 10.06 & -0.89 & 16.60 & 203 & & 7.06 & \\
\hline $0224+683$ & Lan 142 & $17,830(474)$ & $8.19(0.10)$ & $<-4.88(0.49)$ & $0.71(0.06)$ & 11.18 & -1.95 & 17.78 & 209 & & 8.21 & \\
\hline $0249+346$ & KUV 02499+3442 & $13,320(454)$ & $8.96(0.22)$ & $<-5.04(0.41)$ & $1.17(0.10)$ & 13.24 & -3.00 & 16.40 & 42 & & 9.13 & \\
\hline $0249-052$ & KUV 02498-0515 & $17,700(548)$ & $8.16(0.09)$ & $-5.47(0.59)$ & $0.69(0.05)$ & 11.13 & -1.94 & 16.60 & 123 & & 8.20 & \\
\hline $0258+683$ & Lan 143 & $14,650(370)$ & $8.33(0.11)$ & $-3.94(0.04)$ & $0.80(0.07)$ & 11.85 & -2.37 & 16.80 & 97 & & 8.57 & \\
\hline $0300-013$ & GD 40 & $14,780(362)$ & $8.13(0.09)$ & $-6.11(1.00)$ & $0.67(0.06)$ & 11.52 & -2.23 & 15.56 & 64 & & 8.43 & 1 \\
\hline $0308-565$ & L175-34 & $23,000(2336)$ & $8.04(0.07)$ & $<-4.80(3.25)$ & $0.63(0.04)$ & 10.55 & -1.40 & 14.07 & 50 & & 7.64 & 2 \\
\hline $0336+625$ & Lan 174 & $21,280(880)$ & $8.12(0.07)$ & $<-4.05(0.38)$ & $0.67(0.04)$ & 10.74 & -1.58 & 17.15 & 191 & & 7.86 & \\
\hline $0349+015$ & KUV 03493+0131 & $24,860(1939)$ & $7.95(0.07)$ & $<-4.59(1.79)$ & $0.58(0.04)$ & 10.38 & -1.21 & 17.20 & 231 & & 7.39 & \\
\hline $0414-045$ & HE $0414-0434$ & $13,470(335)$ & $8.14(0.11)$ & $-5.60(0.31)$ & $0.67(0.07)$ & 11.76 & -2.40 & 15.70 & 61 & & 8.55 & \\
\hline $0418-539$ & BPM 17731 & $19,050(464)$ & $8.10(0.06)$ & $<-4.58(0.20)$ & $0.66(0.04)$ & 10.90 & -1.77 & 15.32 & 76 & & 8.05 & \\
\hline $0423-145$ & HE $0423-1434$ & $16,900(402)$ & $8.08(0.09)$ & $<-5.98(1.22)$ & $0.64(0.05)$ & 11.11 & -1.97 & 16.21 & 104 & & 8.21 & \\
\hline $0429-168$ & HE $0429-1651$ & $15,540(416)$ & $7.99(0.16)$ & $<-6.35(3.11)$ & $0.59(0.09)$ & 11.19 & -2.06 & 15.82 & 84 & & 8.27 & 2 \\
\hline $0435+410$ & GD 61 & $16,810(410)$ & $8.19(0.09)$ & $-4.20(0.06)$ & $0.70(0.06)$ & 11.28 & -2.04 & 14.86 & 52 & & 8.29 & \\
\hline $0437+138$ & LP $475-242$ & $15,120(362)$ & $8.25(0.09)$ & $-4.68(0.06)$ & $0.74(0.06)$ & 11.64 & -2.27 & 14.92 & 45 & & 8.47 & \\
\hline $0503+147$ & KUV $05034+1445$ & $15,610(382)$ & $8.08(0.08)$ & $-5.38(0.22)$ & $0.64(0.05)$ & 11.31 & -2.11 & 13.80 & 31 & & 8.33 & \\
\hline $0513+260$ & KUV $05134+2605$ & $24,680(1322)$ & $8.21(0.06)$ & $-3.78(0.34)$ & $0.73(0.04)$ & 10.76 & -1.39 & 16.70 & 154 & & 7.66 & 3 \\
\hline $0517+771$ & GD 435 & $13,150(338)$ & $8.13(0.13)$ & $-6.00(0.80)$ & $0.67(0.08)$ & 11.80 & -2.44 & 16.01 & 69 & & 8.57 & \\
\hline $0615-591$ & L182-61 & $15,750(374)$ & $8.04(0.07)$ & $<-6.32(1.09)$ & $0.61(0.04)$ & 11.22 & -2.07 & 14.09 & 37 & & 8.29 & \\
\hline $0716+404$ & GD 85 & $17,150(429)$ & $8.08(0.07)$ & $<-6.07(1.38)$ & $0.64(0.04)$ & 11.08 & -1.94 & 14.94 & 59 & & 8.19 & \\
\hline $0825+367$ & CBS 73 & $15,960(438)$ & $8.12(0.11)$ & $<-5.30(0.38)$ & $0.67(0.07)$ & 11.32 & -2.09 & 17.00 & 136 & & 8.32 & \\
\hline
\end{tabular}


Table 1-Continued

\begin{tabular}{|c|c|c|c|c|c|c|c|c|c|c|c|c|}
\hline WD & Name & $T_{\text {eff }}(\mathrm{K})$ & $\log g$ & $\log \mathrm{H} / \mathrm{He}$ & $M / M_{\odot}$ & $M_{V}$ & $\log L / L_{\odot}$ & $V$ & $D(\mathrm{pc})$ & $1 / V_{\max }$ & $\log \tau$ & Notes \\
\hline $0835+340$ & CSO 197 & $22,290(1382)$ & $8.25(0.07)$ & $<-4.67(2.02)$ & $0.75(0.05)$ & 10.89 & -1.59 & 16.00 & 105 & & 7.91 & \\
\hline $0838+375$ & CBS 78 & $14,280(426)$ & $8.61(0.17)$ & $<-6.49(2.01)$ & $0.97(0.11)$ & 12.39 & -2.60 & 17.71 & 115 & & 8.79 & 1 \\
\hline $0840+262$ & TON 10 & $17,770(421)$ & $8.30(0.07)$ & $-3.98(0.05)$ & $0.78(0.04)$ & 11.32 & -2.01 & 14.78 & 49 & $1.21(-6)$ & 8.30 & \\
\hline $0840+364$ & CBS 82 & $21,260(864)$ & $8.15(0.07)$ & $<-5.05(2.60)$ & $0.69(0.04)$ & 10.80 & -1.61 & 17.03 & 176 & & 7.90 & \\
\hline $0845-188$ & L748-70 & $17,470(420)$ & $8.15(0.08)$ & $<-6.00(1.55)$ & $0.68(0.05)$ & 11.15 & -1.95 & 15.55 & 75 & & 8.21 & 2 \\
\hline $0900+142$ & PG $0900+142$ & $14,860(352)$ & $8.07(0.10)$ & $<-6.44(1.30)$ & $0.63(0.06)$ & 11.42 & -2.19 & 16.48 & 102 & $1.40(-6)$ & 8.39 & 2 \\
\hline $0902+293$ & CBS 3 & $18,590(588)$ & $8.02(0.08)$ & $<-5.47(1.68)$ & $0.61(0.05)$ & 10.82 & -1.76 & 17.00 & 172 & & 8.02 & \\
\hline $0906+341$ & CBS 94 & $17,310(535)$ & $8.06(0.11)$ & $<-5.02(0.52)$ & $0.63(0.07)$ & 11.04 & -1.92 & 17.00 & 155 & & 8.17 & \\
\hline $0921+091$ & PG $0921+092$ & $19,430(522)$ & $8.01(0.07)$ & $-4.63(0.37)$ & $0.60(0.04)$ & 10.73 & -1.68 & 16.19 & 123 & $5.60(-7)$ & 7.94 & \\
\hline $0948+013$ & PG $0948+013$ & $16,810(432)$ & $8.09(0.07)$ & $-5.38(0.29)$ & $0.65(0.04)$ & 11.15 & -1.99 & 15.59 & 77 & $9.75(-7)$ & 8.23 & \\
\hline $0954+342$ & CBS 114 & $26,050(1823)$ & $7.98(0.08)$ & $<-3.99(0.45)$ & $0.60(0.04)$ & 10.37 & -1.15 & 17.20 & 232 & & 7.31 & 3 \\
\hline $1006+413$ & KUV $10064+4120$ & $15,100(537)$ & $8.82(0.20)$ & $<-5.44(0.92)$ & $1.10(0.11)$ & 12.68 & -2.67 & 17.83 & 107 & & 8.93 & \\
\hline $1009+416$ & KUV $10098+4138$ & $16,480(424)$ & $8.65(0.08)$ & $<-5.20(0.30)$ & $1.00(0.05)$ & 12.13 & -2.39 & 16.33 & 69 & & 8.65 & \\
\hline $1011+570$ & GD 303 & $17,350(424)$ & $8.13(0.07)$ & $<-5.01(0.21)$ & $0.67(0.04)$ & 11.13 & -1.95 & 14.57 & 48 & & 8.21 & \\
\hline $1026-056$ & PG $1026-057$ & $17,650(427)$ & $8.08(0.06)$ & $<-4.93(0.22)$ & $0.64(0.04)$ & 11.02 & -1.89 & 16.94 & 153 & $8.18(-7)$ & 8.15 & \\
\hline $1046-017$ & GD 124 & $14,620(354)$ & $8.14(0.13)$ & $<-6.46(1.63)$ & $0.68(0.08)$ & 11.57 & -2.26 & 15.81 & 70 & $1.71(-6)$ & 8.45 & 2 \\
\hline $1056+345$ & G119-47 & $12,440(337)$ & $8.23(0.15)$ & $-5.30(0.22)$ & $0.73(0.10)$ & 12.09 & -2.60 & 15.58 & 49 & $3.62(-6)$ & 8.70 & \\
\hline $1107+265$ & GD 128 & $15,060(361)$ & $8.09(0.08)$ & $-5.36(0.18)$ & $0.65(0.05)$ & 11.42 & -2.18 & 15.89 & 78 & $1.39(-6)$ & 8.38 & \\
\hline $1115+158$ & PG $1115+158$ & $23,770(1624)$ & $7.91(0.07)$ & $-3.84(0.41)$ & $0.56(0.04)$ & 10.35 & -1.26 & 16.12 & 142 & $3.39(-7)$ & 7.44 & 3 \\
\hline $1129+373$ & PG $1129+373$ & $13,030(358)$ & $8.16(0.16)$ & $-6.06(1.17)$ & $0.68(0.10)$ & 11.86 & -2.47 & 16.23 & 74 & $2.63(-6)$ & 8.60 & \\
\hline $1144-084$ & PG $1144-085$ & $15,730(379)$ & $8.06(0.08)$ & $<-6.32(1.37)$ & $0.63(0.04)$ & 11.27 & -2.08 & 15.95 & 86 & $1.15(-6)$ & 8.31 & 2 \\
\hline $1148+408$ & KUV $11489+4052$ & $17,130(443)$ & $8.30(0.11)$ & $<-4.66(0.24)$ & $0.78(0.07)$ & 11.43 & -2.08 & 17.33 & 151 & & 8.35 & \\
\hline $1149-133$ & PG $1149-133$ & $20,370(578)$ & $8.30(0.06)$ & $-3.82(0.14)$ & $0.78(0.04)$ & 11.07 & -1.77 & 16.29 & 110 & $8.59(-7)$ & 8.11 & \\
\hline $1252-289$ & EC $12522-2855$ & $21,880(757)$ & $8.03(0.06)$ & $<-4.85(1.24)$ & $0.62(0.03)$ & 10.59 & -1.49 & 15.85 & 112 & & 7.74 & \\
\hline $1311+129$ & LP 497-114 & $19,100(479)$ & $7.96(0.07)$ & $-2.90(0.06)$ & $0.58(0.04)$ & 10.64 & -1.68 & 16.26 & 132 & $5.09(-7)$ & 7.92 & \\
\hline $1326-037$ & PG $1326-037$ & $19,890(531)$ & $8.03(0.06)$ & $<-4.66(0.39)$ & $0.62(0.04)$ & 10.72 & -1.65 & 15.60 & 94 & $5.51(-7)$ & 7.92 & \\
\hline $1332+162$ & PB 3990 & $16,790(424)$ & $8.17(0.08)$ & $-5.09(0.26)$ & $0.70(0.05)$ & 11.28 & -2.04 & 15.98 & 87 & $1.15(-6)$ & 8.28 & \\
\hline $1333+487$ & GD 325 & $15,320(377)$ & $8.03(0.09)$ & $<-5.40(0.26)$ & $0.61(0.05)$ & 11.28 & -2.11 & 14.02 & 35 & $1.17(-6)$ & 8.32 & \\
\hline $1336+123$ & LP 498-26 & $15,950(407)$ & $8.01(0.09)$ & $<-6.29(1.90)$ & $0.60(0.05)$ & 11.16 & -2.03 & 14.72 & 51 & $9.92(-7)$ & 8.25 & 2 \\
\hline
\end{tabular}


Table 1-Continued

\begin{tabular}{|c|c|c|c|c|c|c|c|c|c|c|c|c|}
\hline WD & Name & $T_{\text {eff }}(\mathrm{K})$ & $\log g$ & $\log \mathrm{H} / \mathrm{He}$ & $M / M_{\odot}$ & $M_{V}$ & $\log L / L_{\odot}$ & $V$ & $D(\mathrm{pc})$ & $1 / V_{\max }$ & $\log \tau$ & Notes \\
\hline $1351+489$ & PG $1351+489$ & $26,010(1536)$ & $7.91(0.07)$ & $<-4.37(0.82)$ & $0.56(0.04)$ & 10.27 & -1.11 & 16.38 & 166 & & 7.27 & 3,4 \\
\hline $1352+004$ & PG $1352+004$ & $13,980(341)$ & $8.05(0.10)$ & $-5.30(0.16)$ & $0.62(0.06)$ & 11.53 & -2.28 & 15.72 & 69 & $1.64(-6)$ & 8.45 & \\
\hline $1403-010$ & G64-43 & $15,420(375)$ & $8.10(0.08)$ & $-6.10(0.94)$ & $0.65(0.05)$ & 11.37 & -2.14 & 15.90 & 80 & $1.30(-6)$ & 8.35 & \\
\hline $1411+218$ & PG $1411+219$ & $14,910(361)$ & $8.04(0.09)$ & $<-5.46(0.25)$ & $0.62(0.05)$ & 11.36 & -2.17 & 14.30 & 38 & $1.30(-6)$ & 8.36 & \\
\hline $1415+234$ & PG $1415+234$ & $17,380(469)$ & $8.19(0.08)$ & $-5.06(0.34)$ & $0.71(0.05)$ & 11.23 & -1.99 & 16.80 & 130 & $1.07(-6)$ & 8.25 & \\
\hline $1416+229$ & KUV $14161+2255$ & $17,410(437)$ & $8.22(0.10)$ & $<-4.67(0.23)$ & $0.73(0.06)$ & 11.26 & -2.00 & 16.60 & 116 & & 8.27 & \\
\hline $1419+351$ & GD 335 & $12,830(585)$ & $8.88(0.36)$ & $<-5.58(1.46)$ & $1.13(0.18)$ & 13.15 & -3.00 & 16.89 & 55 & & 9.14 & \\
\hline $1421-011$ & PG $1421-011$ & $16,910(413)$ & $8.20(0.09)$ & $-4.26(0.07)$ & $0.71(0.06)$ & 11.28 & -2.04 & 15.97 & 86 & $1.15(-6)$ & 8.29 & \\
\hline $1425+540$ & G200-39 & $14,490(345)$ & $7.95(0.08)$ & $-4.20(0.03)$ & $0.56(0.05)$ & 11.29 & -2.16 & 15.04 & 56 & $1.20(-6)$ & 8.33 & \\
\hline $1444-096$ & PG 1444-096 & $17,040(412)$ & $8.26(0.11)$ & $-5.82(1.21)$ & $0.75(0.07)$ & 11.38 & -2.06 & 14.98 & 52 & $1.30(-6)$ & 8.32 & \\
\hline $1445+152$ & PG $1445+153$ & $20,960(840)$ & $8.05(0.08)$ & $<-5.12(2.62)$ & $0.63(0.04)$ & 10.66 & -1.57 & 15.55 & 95 & $5.08(-7)$ & 7.84 & \\
\hline $1454-630.1$ & L151-81A & 14,050 & $7.96(0.09)$ & $-4.79(0.06)$ & $0.57(0.05)$ & 11.39 & -2.23 & 16.60 & 110 & & 8.38 & \\
\hline $1456+103$ & PG $1456+103$ & $24,080(1199)$ & $7.91(0.08)$ & $-3.24(0.14)$ & $0.56(0.04)$ & 10.32 & -1.24 & 15.89 & 129 & $3.27(-7)$ & 7.41 & 3 \\
\hline $1459+821$ & G256-18 & $15,850(395)$ & $8.09(0.08)$ & $<-5.32(0.24)$ & $0.65(0.05)$ & 11.29 & -2.09 & 14.78 & 49 & & 8.31 & \\
\hline $1540+680$ & PG $1540+681$ & $22,140(1246)$ & $7.96(0.07)$ & $<-4.25(0.62)$ & $0.58(0.04)$ & 10.47 & -1.42 & 16.19 & 139 & $3.99(-7)$ & 7.64 & \\
\hline $1542+182$ & GD 190 & $22,630(984)$ & $8.04(0.06)$ & $<-4.84(1.41)$ & $0.63(0.03)$ & 10.57 & -1.43 & 14.72 & 67 & $4.48(-7)$ & 7.67 & \\
\hline $1542-275$ & LP 916-27 & $12,700(385)$ & $9.13(0.15)$ & $-4.95(0.58)$ & $1.24(0.06)$ & 13.72 & -3.23 & 15.49 & 22 & & 9.19 & \\
\hline $1545+244$ & Ton 249 & $12,840(332)$ & $8.18(0.13)$ & $-5.02(0.11)$ & $0.70(0.08)$ & 11.93 & -2.51 & 15.78 & 58 & $2.88(-6)$ & 8.63 & \\
\hline $1551+175$ & KUV 15519+1730 & $15,550(378)$ & $7.96(0.11)$ & $-4.38(0.07)$ & $0.57(0.06)$ & 11.13 & -2.04 & 17.50 & 188 & & 8.24 & 5 \\
\hline $1557+192$ & KUV 15571+1913 & $19,570(558)$ & $8.15(0.07)$ & $-4.37(0.30)$ & $0.68(0.04)$ & 10.92 & -1.75 & 15.40 & 78 & & 8.04 & \\
\hline $1610+239$ & PG $1610+239$ & $13,360(334)$ & $8.16(0.12)$ & $<-5.58(0.30)$ & $0.69(0.07)$ & 11.81 & -2.43 & 15.34 & 50 & $2.42(-6)$ & 8.57 & \\
\hline $1612-111$ & GD 198 & $23,420(1781)$ & $7.96(0.06)$ & $<-4.75(2.11)$ & $0.59(0.04)$ & 10.44 & -1.32 & 15.53 & 104 & & 7.53 & \\
\hline $1644+198$ & PG $1644+199$ & $15,190(383)$ & $8.12(0.10)$ & $<-5.42(0.31)$ & $0.66(0.06)$ & 11.43 & -2.18 & 15.20 & 56 & $1.41(-6)$ & 8.38 & \\
\hline $1645+325$ & GD 358 & $24,940(1115)$ & $7.92(0.06)$ & $<-4.58(0.88)$ & $0.57(0.03)$ & 10.33 & -1.19 & 13.65 & 46 & $3.31(-7)$ & 7.36 & 3 \\
\hline $1654+160$ & PG $1654+160$ & $29,410(1613)$ & $7.97(0.08)$ & $<-3.98(1.19)$ & $0.60(0.04)$ & 10.18 & -0.92 & 16.55 & 187 & $2.62(-7)$ & 7.07 & 3 \\
\hline $1703+319$ & PG $1703+319$ & $14,430(362)$ & $8.45(0.11)$ & $-5.51(0.34)$ & $0.88(0.07)$ & 12.09 & -2.48 & 16.25 & 67 & $3.38(-6)$ & 8.67 & \\
\hline $1708-871$ & L7-44 & $23,980(1686)$ & $8.05(0.06)$ & $<-4.69(1.93)$ & $0.64(0.03)$ & 10.54 & -1.33 & 14.38 & 58 & & 7.55 & \\
\hline $1709+230$ & GD 205 & $19,610(507)$ & $8.09(0.06)$ & $-4.00(0.13)$ & $0.65(0.04)$ & 10.81 & -1.71 & 14.90 & 65 & & 7.99 & \\
\hline $1726-578$ & L204-118 & $14,320(341)$ & $8.20(0.08)$ & $-5.46(0.19)$ & $0.71(0.05)$ & 11.70 & -2.33 & 15.27 & 51 & & 8.51 & \\
\hline $1822+410$ & GD 378 & $16,230(385)$ & $8.01(0.08)$ & $-4.45(0.06)$ & $0.60(0.05)$ & 11.09 & -2.00 & 14.39 & 45 & & 8.22 & \\
\hline
\end{tabular}


Table 1-Continued

\begin{tabular}{|c|c|c|c|c|c|c|c|c|c|c|c|c|}
\hline WD & Name & $T_{\text {eff }}(\mathrm{K})$ & $\log g$ & $\log \mathrm{H} / \mathrm{He}$ & $M / M_{\odot}$ & $M_{V}$ & $\log L / L_{\odot}$ & $V$ & $D(\mathrm{pc})$ & $1 / V_{\max }$ & $\log \tau$ & Notes \\
\hline $1940+374$ & L1573-31 & $16,630(431)$ & $8.07(0.08)$ & $<-5.17(0.28)$ & $0.64(0.05)$ & 11.14 & -1.99 & 14.51 & 47 & & 8.23 & \\
\hline $2034-532$ & L279-25 & $17,160(406)$ & $8.48(0.07)$ & $<-5.74(0.57)$ & $0.90(0.05)$ & 11.73 & -2.19 & 14.46 & 35 & & 8.49 & \\
\hline $2058+342$ & GD 392A & $12,220(423)$ & $9.09(0.20)$ & $<-5.54(2.56)$ & $1.23(0.08)$ & 13.72 & -3.26 & 15.68 & 24 & & 9.22 & \\
\hline $2129+000$ & G26-10 & $14,380(351)$ & $8.26(0.14)$ & $<-6.48(1.66)$ & $0.75(0.09)$ & 11.79 & -2.36 & 15.27 & 49 & & 8.55 & 2 \\
\hline $2130-047$ & GD 233 & $18,110(427)$ & $8.11(0.08)$ & $-5.76(1.32)$ & $0.66(0.05)$ & 11.01 & -1.86 & 14.52 & 50 & & 8.13 & \\
\hline $2144-079$ & G26-31 & $16,340(410)$ & $8.18(0.07)$ & $<-6.22(1.45)$ & $0.70(0.04)$ & 11.36 & -2.09 & 14.82 & 49 & & 8.33 & 2 \\
\hline $2222+683$ & G241-6 & $15,230(380)$ & $8.20(0.11)$ & $<-5.42(0.43)$ & $0.71(0.07)$ & 11.56 & -2.23 & 15.65 & 65 & & 8.43 & 1 \\
\hline $2229+139$ & PG $2229+139$ & $14,940(357)$ & $8.18(0.09)$ & $-4.73(0.07)$ & $0.70(0.05)$ & 11.57 & -2.25 & 15.99 & 76 & $1.70(-6)$ & 8.44 & \\
\hline $2234+064$ & PG $2234+064$ & $23,770(1770)$ & $8.07(0.06)$ & $<-4.72(2.10)$ & $0.65(0.04)$ & 10.58 & -1.36 & 16.03 & 123 & $4.51(-7)$ & 7.59 & \\
\hline $2236+541$ & KPD $2236+5410$ & $15,470(386)$ & $8.30(0.09)$ & $<-5.38(0.27)$ & $0.78(0.06)$ & 11.68 & -2.26 & 16.19 & 79 & & 8.48 & \\
\hline $2246+120$ & PG $2246+121$ & $27,070(1521)$ & $7.92(0.07)$ & $<-4.27(0.67)$ & $0.57(0.04)$ & 10.23 & -1.04 & 16.73 & 199 & $2.86(-7)$ & 7.20 & 3 \\
\hline $2250+746$ & GD 554 & $16,370(386)$ & $8.16(0.06)$ & $<-5.22(0.12)$ & $0.69(0.04)$ & 11.32 & -2.07 & 16.69 & 118 & & 8.31 & \\
\hline $2253-062$ & GD 243 & $17,190(437)$ & $8.07(0.10)$ & $-4.33(0.12)$ & $0.64(0.06)$ & 11.05 & -1.93 & 15.06 & 63 & & 8.18 & \\
\hline $2310+175$ & KUV 23103+1736 & $15,170(373)$ & $8.37(0.09)$ & $<-5.43(0.26)$ & $0.82(0.06)$ & 11.84 & -2.34 & 15.88 & 64 & $2.40(-6)$ & 8.56 & \\
\hline $2316-173$ & G273-13 & $12,610(424)$ & $9.11(0.19)$ & $-4.85(0.56)$ & $1.23(0.07)$ & 13.70 & -3.23 & 14.08 & 11 & & 9.19 & \\
\hline $2328+510$ & GD 406 & $14,460(362)$ & $8.06(0.12)$ & $<-5.51(0.35)$ & $0.63(0.07)$ & 11.47 & -2.23 & 15.09 & 53 & & 8.42 & \\
\hline $2354+159$ & PG $2354+159$ & $24,830(1671)$ & $8.15(0.06)$ & $<-4.59(1.78)$ & $0.70(0.04)$ & 10.67 & -1.34 & 15.78 & 105 & $5.00(-7)$ & 7.57 & \\
\hline
\end{tabular}

Note. - (1) Solution with $\log \mathrm{Ca} / \mathrm{He}=-7.0 ;(2)$ Limits on the hydrogen abundance based on the absence of H $\alpha$ from Table 1 of Voss et al. 2007; (3) Variable white dwarf of the V777 Her class; (4) PG star not in the complete sample; (5) Solution with log Ca/He $=-6.5$. 
Table 2. Parameters of DB Stars with Trigonometric Parallaxes

\begin{tabular}{clcccccccc}
\hline \hline WD & Name & $\begin{array}{c}\pi \\
(\mathrm{mas})\end{array}$ & $\begin{array}{c}\sigma_{\pi} \\
(\mathrm{mas})\end{array}$ & $\begin{array}{c}D_{\pi} \\
(\mathrm{pc})\end{array}$ & $\begin{array}{c}D_{\text {spec }} \\
(\mathrm{pc})\end{array}$ & $\begin{array}{c}T_{\text {eff }} \\
(\mathrm{K})\end{array}$ & $\log g$ & $M / M_{\odot}$ & Notes \\
\hline $0002+729$ & GD 408 & 28.80 & $(4.7)$ & 34.7 & 32.2 & 14,410 & 8.26 & 0.75 & 1 \\
$0017+136$ & Feige 4 & 30.00 & $(9.4)$ & 33.3 & 75.9 & 18,130 & 8.09 & 0.65 & 1 \\
$0615-591$ & L182-61 & 27.50 & $(1.0)$ & 36.4 & 37.5 & 15,750 & 8.04 & 0.61 & 2 \\
$1333+487$ & GD 325 & 28.60 & $(3.2)$ & 35.0 & 35.3 & 15,320 & 8.03 & 0.61 & 1 \\
$1425+540$ & G200-39 & 17.30 & $(3.9)$ & 57.8 & 56.2 & 14,490 & 7.95 & 0.56 & 1 \\
$1645+325$ & GD 358 & 27.30 & $(3.3)$ & 36.6 & 46.0 & 24,940 & 7.92 & 0.57 & 1 \\
$1940+374$ & L1573-31 & 20.30 & $(2.8)$ & 49.3 & 47.2 & 16,630 & 8.07 & 0.64 & 1 \\
$2129+000$ & G26-10 & 20.26 & $(2.0)$ & 49.4 & 49.6 & 14,380 & 8.26 & 0.75 & 2 \\
$2144-079$ & G26-31 & 14.40 & $(5.8)$ & 69.4 & 49.2 & 16,340 & 8.18 & 0.70 & 1 \\
$2147+280$ & G188-27 & 28.30 & $(3.0)$ & 35.3 & 20.8 & 12,940 & 8.85 & 1.12 & 1 \\
$2222+683$ & G241-6 & 14.70 & $(4.4)$ & 68.0 & 65.8 & 15,230 & 8.20 & 0.71 & 3 \\
\hline
\end{tabular}

Note. - (1) YPC, (2) Gould \& Chanamé 2004, (3) Dahn et al. 1988. 
Table 3. White Dwarf Space Density ${ }^{\mathrm{a}}$ in the PG Survey

\begin{tabular}{cccc}
\hline \hline$M_{\text {bol }}$ & DB & DA & Total \\
\hline 6 & - & $2.43 \times 10^{-6}$ & $2.43 \times 10^{-6}$ \\
7 & $7.28 \times 10^{-7}$ & $1.23 \times 10^{-5}$ & $1.30 \times 10^{-5}$ \\
8 & $3.10 \times 10^{-6}$ & $2.48 \times 10^{-5}$ & $2.79 \times 10^{-5}$ \\
9 & $4.57 \times 10^{-6}$ & $5.15 \times 10^{-5}$ & $5.61 \times 10^{-5}$ \\
10 & $2.57 \times 10^{-5}$ & $6.00 \times 10^{-5}$ & $8.58 \times 10^{-5}$ \\
11 & $1.73 \times 10^{-5}$ & $6.11 \times 10^{-5}$ & $7.85 \times 10^{-5}$ \\
\hline
\end{tabular}

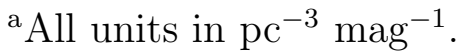


Fig. 1. - Left panel: synthetic spectra of pure helium DB models ( $\log g=8$ and ML2/ $\alpha=$ 1.25) at various effective temperatures (blue) normalized to a continuum set to unity and offset from each other by a factor of 0.4 for clarity; each model spectrum is compared to a spectrum at $T_{\text {eff }}=24,000 \mathrm{~K}$ (red). Middle panel: same as left panel but for synthetic spectra at $\log g=7.5$ (red) and $\log g=8.5$ (blue). Right panel: same as left panels but for synthetic spectra calculated with ML2/ $\alpha=0.75$ (red) and $\alpha=1.75$ (blue).

Fig. 2.- Variation of the equivalent width of He I $\lambda 4471$ (measured from 4220 to $4625 \AA$ ) as a function of effective temperature for our grid of model spectra with various hydrogen abundances (left panel), surface gravities (middle panel), and convective efficiencies (right panel). Also shown as red circles are the measured equivalent widths in our sample of DB stars, arbitrarily located at $T_{\text {eff }}=22,000 \mathrm{~K}$.

Fig. 3.- Location of the helium convection zone (hatched region) as a function of effective temperature in the pure helium envelope of a $0.6 M_{\odot}$ DB white dwarf calculated with the ML2/ $\alpha=0.6$ version of the mixing-length theory (from G. Fontaine \& P. Brassard 2006, private communication). The depth is expressed as the fractional mass above the point of interest with respect to the total mass of the star. The thick solid line corresponds to the photosphere $\left(\tau_{R} \sim 1\right)$.

Fig. 4.- Distribution of DB stars in our sample as a function of the $V$ magnitude.

Fig. 5.- Optical (blue) spectra for our complete sample of DB stars. The spectra are normalized at $4500 \AA$ and shifted vertically from each other by a factor of 0.5 for clarity. The effective temperature decreases from upper left to bottom right.

Fig. 6. - Distribution of signal-to-noise ratios for the 108 optical spectra secured for the analysis of the DB stars in our sample (hatched histogram). Also shown are the corresponding values for 744 DB stars identified in the Data Release 4 of the SDSS (thick solid line).

Fig. 7.- Misclassified spectra in our original target list of DB white dwarfs.

Fig. 8.- Optical spectra in the red for the DB stars in our sample, when available, ordered in right ascension. The spectra are normalized at $6200 \AA$ and shifted vertically from each other by a factor of 0.5 for clarity. The dotted line indicates the location of $\mathrm{H} \alpha$. Spectra labeled in red are taken from the SDSS. $\mathrm{H} \alpha$ spectra from the SPY survey, also used in our analysis of DBA stars, are displayed in Voss et al. (2007).

Fig. 9.- Example of the procedure used to define the continuum. In the top panel, the observed spectrum is fitted with a model spectrum multiplied by a high order polynomial (up 
to $\lambda^{5}$ ) to achieve the best possible match (shown in red); in this case the resulting atmospheric parameters are meaningless since too many free parameters are used in the fitting procedure. The continuum flux is then defined by this fitting function at some predefined wavelength points (shown as blue tick marks in the bottom panel), and these are then used to normalize each segment of the spectrum to a continuum set to unity, as shown in the bottom panel.

Fig. 10.- Examples of spectroscopic fits for two DB white dwarfs using a cool (bottom panels) and a hot (top panels) initial estimate of the effective temperature in our fitting procedure.

Fig. 11. - Example of a full spectroscopic fit where the $\mathrm{H} \alpha$ line profile, shown in the insert, is used to measure, or constrain, the hydrogen abundance of the overall solution.

Fig. 12. - Limits on the hydrogen abundance set by our spectroscopic observations at $\mathrm{H} \alpha$ and $H \beta$. We estimate the limit of detectability at an equivalent width of $200 \mathrm{~m} \AA$ and 300 $\mathrm{m} \AA$, respectively.

Fig. 13.- Our best fits to the blue spectra of the strongest DBZ stars in our sample. The region near $\mathrm{H} \alpha$ is not displayed here.

Fig. 14. - Distribution of surface gravity as a function of effective temperature for all DB white dwarfs in our sample for various parameterization of the convective efficiency. Also shown in each panel is a $0.6 M_{\odot}$ evolutionary sequence.

Fig. 15.- Top two panels: Comparison of optical and UV temperatures for 34 DB stars in our sample with available $I U E$ spectra, using two different parameterization of the convective efficiency. Bottom panel: UV temperatures obtained by Castanheira et al. (2006) based on ML2 $/ \alpha=0.6$ models compared with our own temperature estimates based on similar models.

Fig. 16. - Comparison of UV temperatures obtained from model spectra calculated with different convective efficiencies (top panel), different hydrogen abundances (middle panel), and different surface gravities (bottom panel). In each panel, the UV temperatures on the $\mathrm{y}$-axis, obtained from models with parameters given in the legend, are compared against UV temperatures obtained from pure helium model atmospheres at $\log g=8$ and ML2 $/ \alpha=1.25$.

Fig. 17.- Comparison of $T_{\text {eff }}$ and $\log g$ determinations for 28 DB stars in our sample with mutliple spectroscopic observations.

Fig. 18. - Sample fits for several DB and DBA stars in our sample; the atmospheric parameters $\left(T_{\text {eff }}, \log g\right.$, and $\left.\log \mathrm{H} / \mathrm{He}\right)$ of each object are given in the figure. The region near $\mathrm{H} \alpha$ (right panel) is used to measure, or constrain, the hydrogen abundance. In the case of 
DB stars, these high signal-to-noise spectra provide upper limits on the hydrogen-to-helium abundance ratio.

Fig. 19. - Comparison of $T_{\text {eff }}$ and $\log g$ measurements between our analysis and that of Voss et al. (2007) as a function of our $T_{\text {eff }}$ values (this work - Voss in each case). The dashed lines represent a perfect agreement. Objects shown as open circles correspond to cool DB white dwarfs for which Voss et al. assumed a value of $\log g=8$. The two objects labeled in the figure correspond to (1) PG 1115+128 and (2) KUV 03493+0131 (0349+015).

Fig. 20.- Spectroscopic solutions obtained by Voss et al. (2007) for PG 1115+158 and KUV 03493+0131. These should be contrasted with our solutions displayed in Figures 11 and 18 , respectively.

Fig. 21. - Distribution of mass as a function of effective temperature for the 61 DB (red symbols) and 47 DBA (blue symbols) stars in our sample. Also shown as dotted lines are the theoretical isochrones from our evolutionary models, labeled as $\log \tau$ where $\tau$ is the white dwarf cooling age in years.

Fig. 22.- Comparison of distances inferred from trigonometric parallax measurements $\left(D_{\pi}\right)$ with those derived from spectroscopy $\left(D_{\text {spec }}\right)$ taken from Table 2 . The objects labeled in the figure correspond to (1) Feige $4(0017+136)$, (2) GD $358(1645+325)$, and (3) G188-27 $(2147+280)$.

Fig. 23. - Mass distribution of all DB and DBA white dwarfs in our sample (hatched histogram). If the most massive DB stars in this distribution are excluded (see text), the mean mass becomes of $\langle M\rangle=0.671 M_{\odot}$ with a standard deviation of $\sigma_{M}=0.091 M_{\odot}$. Also shown as a thick solid line is the mass distribution of the DA stars in the PG sample.

Fig. 24.- Luminosity function derived for all DB and DA stars from the complete PG sample presented in bolometric magnitude bins, assuming a scale height for the Galaxy of $z_{0}=250$ pc (see Liebert et al. 2005 for details).

Fig. 25.- Hydrogen-to-helium abundance ratio as a function of effective temperature for all DB (white symbols) and DBA (red symbols) white dwarfs in our sample. Limits on the hydrogen abundance set by our spectroscopic observations at $\mathrm{H} \alpha$ (lower dotted line) and $\mathrm{H} \beta$ (upper dotted line) are reproduced from Figure 12. The hydrogen abundances for DB stars represent only upper limits.

Fig. 26. - Total hydrogen mass as a function of effective temperature for all DB (white symbols) and DBA (red symbols) in our sample. The values for DB stars represent only upper limits. 
Fig. 27.- Same as Figure 25, but including also the results for DZA stars from Dufour et al. (2007a). Solid lines represent the expected abundances for continuous accretion of material from the interstellar medium with accretion rates of $10^{-21}$ to $10^{-17} M_{\odot} \mathrm{yr}^{-1}$.

Fig. 28.- Our best fit to three independent spectroscopic observations of LP 497-114 $(1311+129)$, the DBA star with the largest hydrogen abundance in our sample. Our adopted solution is derived from the bottom spectrum, obtained in 2010. The other spectra have been secured in 1995 (middle) and 2004 (top; from SDSS).

Fig. 29.- Location of the V777 Her stars in a mass versus effective temperature diagram. Variable DB white dwarfs are represented by filled symbols while open symbols correspond to photometrically constant, or unknown, objects. DB and DBA stars are shown in black and red, respectively. The dashed lines correspond to theoretical blue edges for pure helium envelope models with convective efficiencies given by, from left to right, ML3, ML2, and ML1.

Fig. 30.- Our best fit to HE 2149-0516 assuming composite DA + DB models. The atmospheric parameters of each component are given in the figure; the $\log g$ values have been kept constant in the fitting procedure, and a hydrogen abundance of $\mathrm{H} / \mathrm{He}=10^{-6}$ was also assumed for the DB component. Note that the relative flux scale starts at 0.4 so the absorption lines are particularly weak as a result of the dilution by each component of the system. 


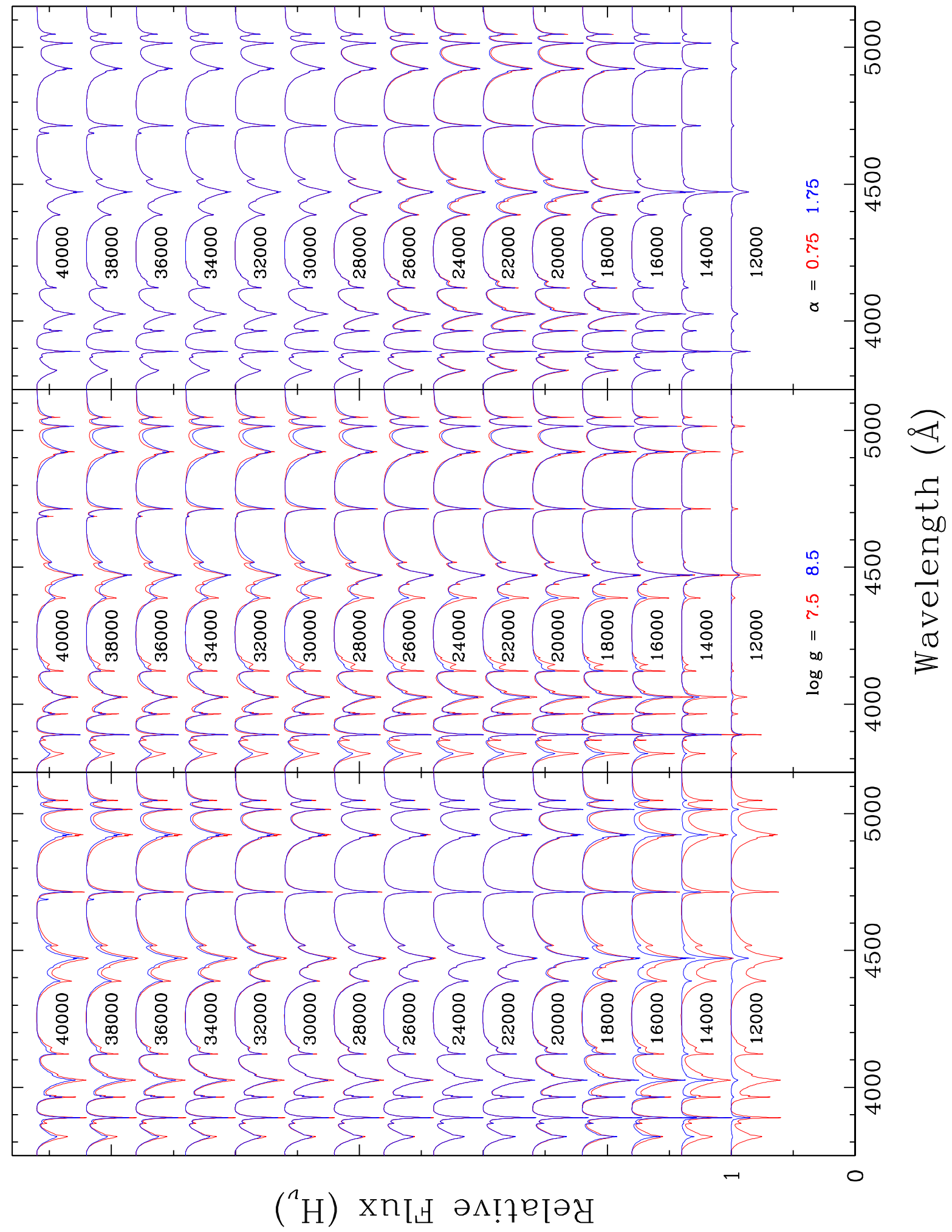

Figure 1 


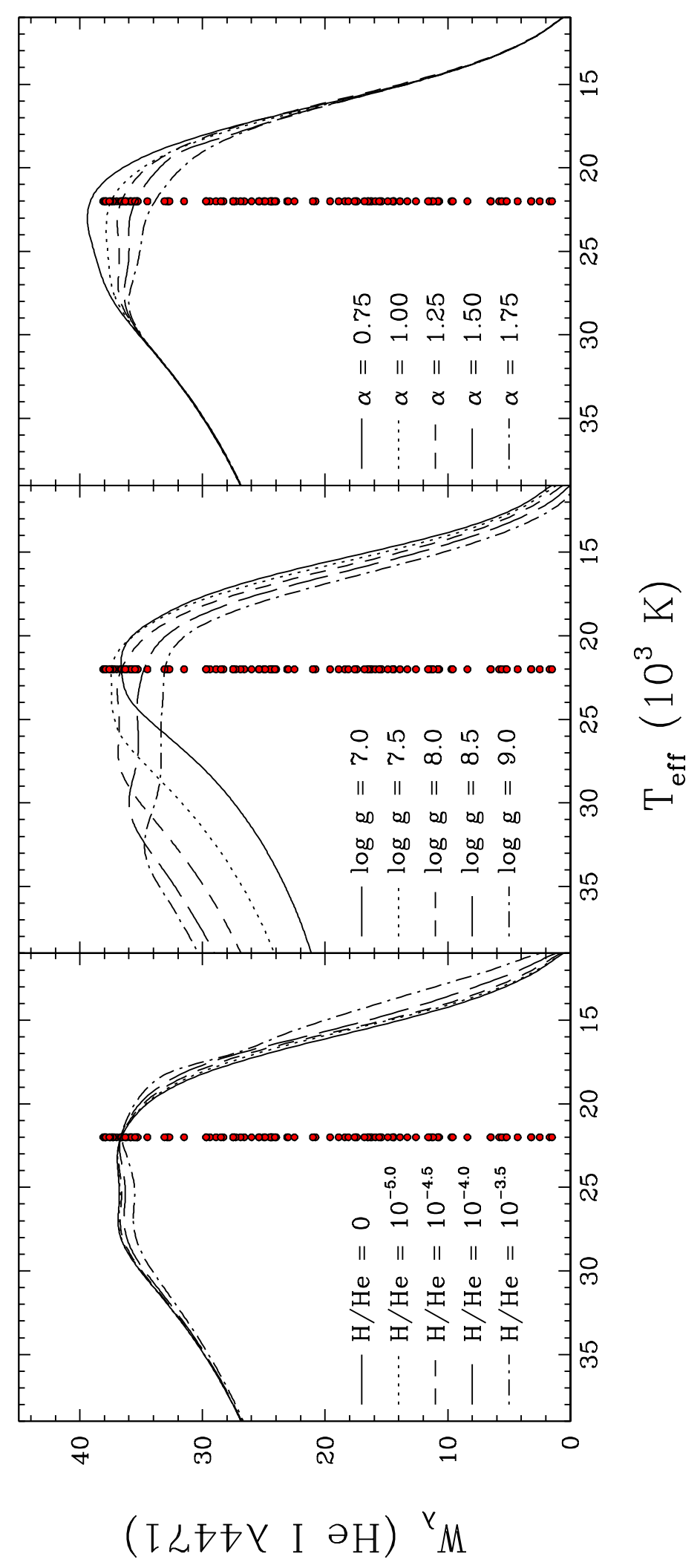

Figure 2 


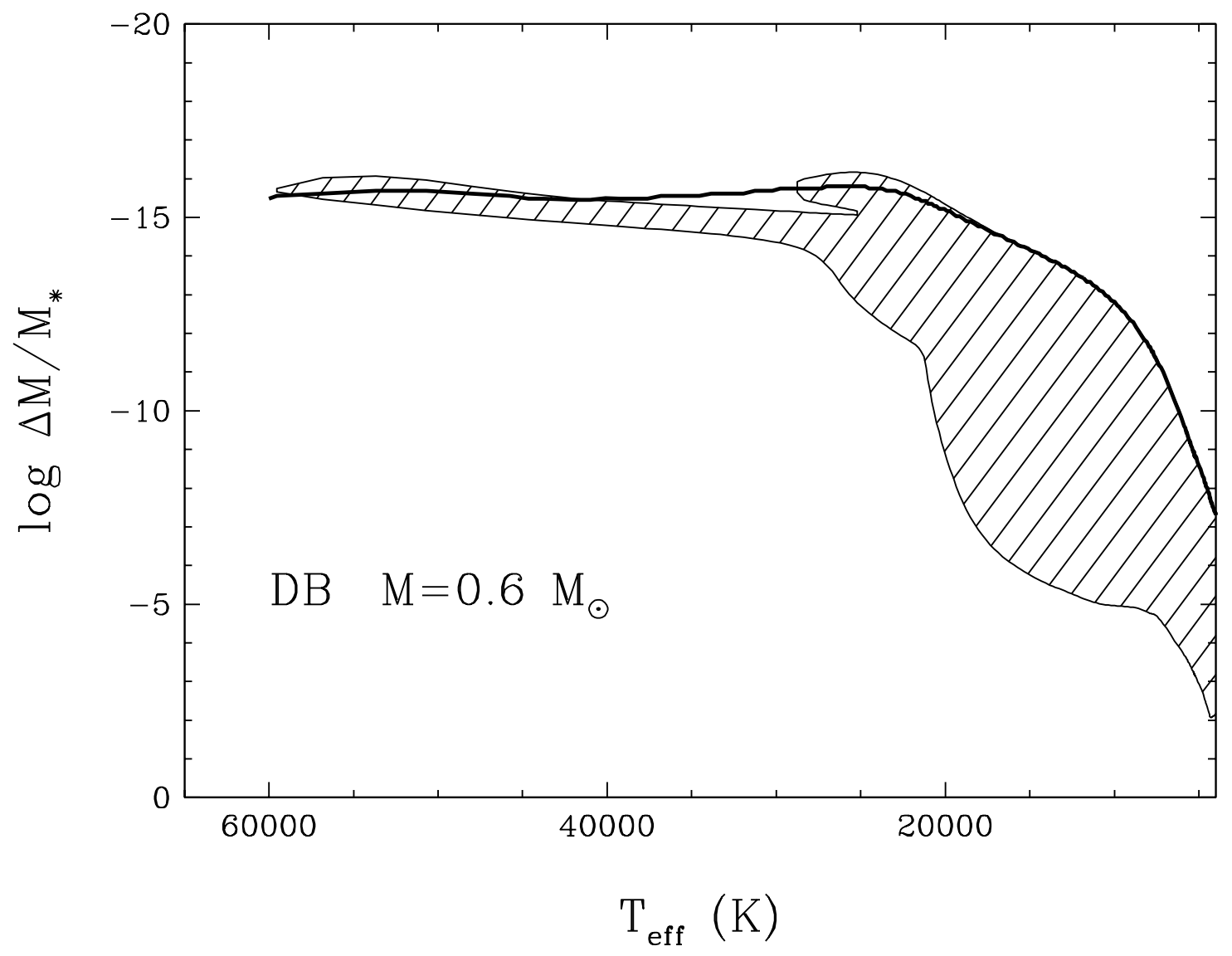

Figure 3 


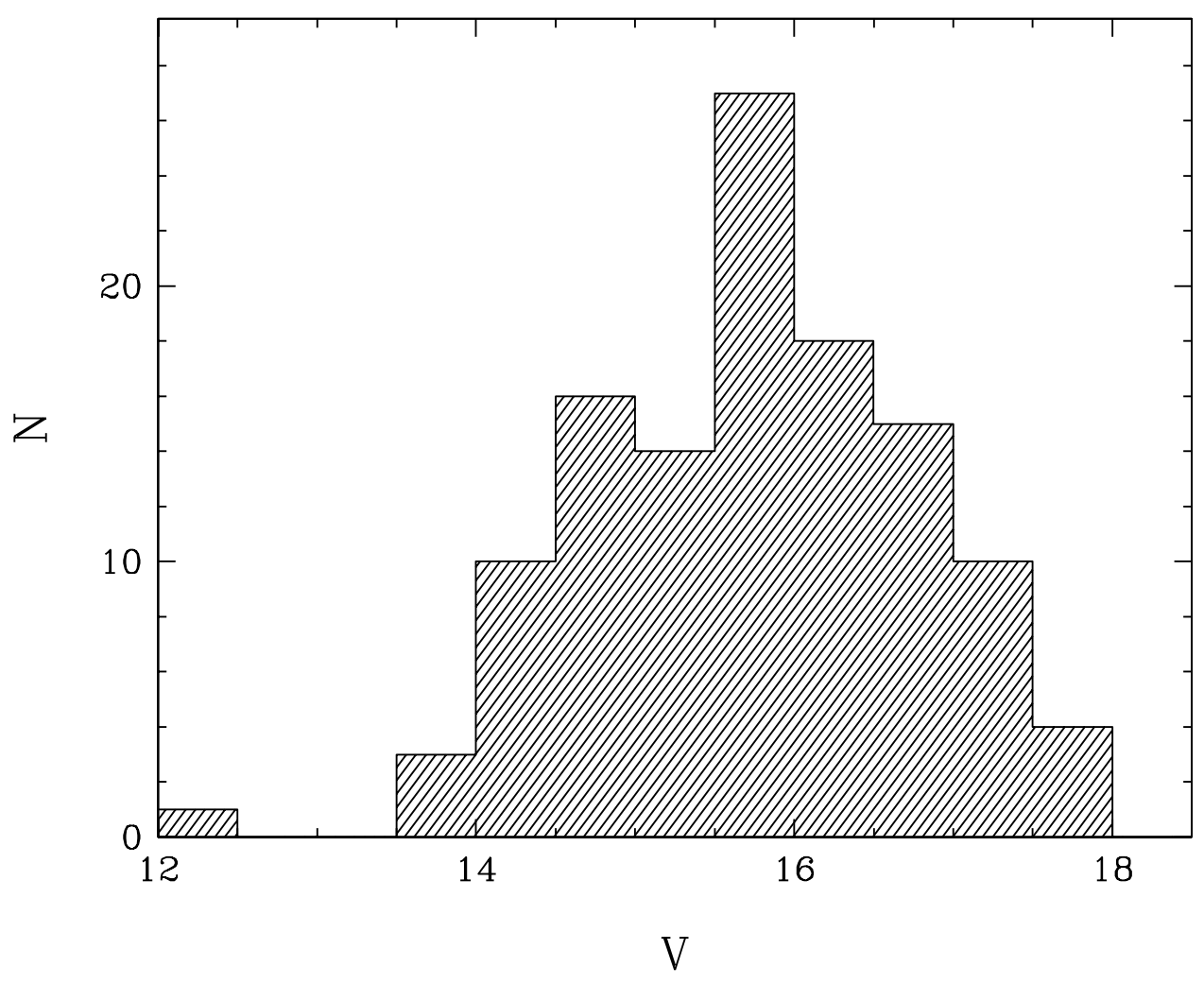

Figure 4 


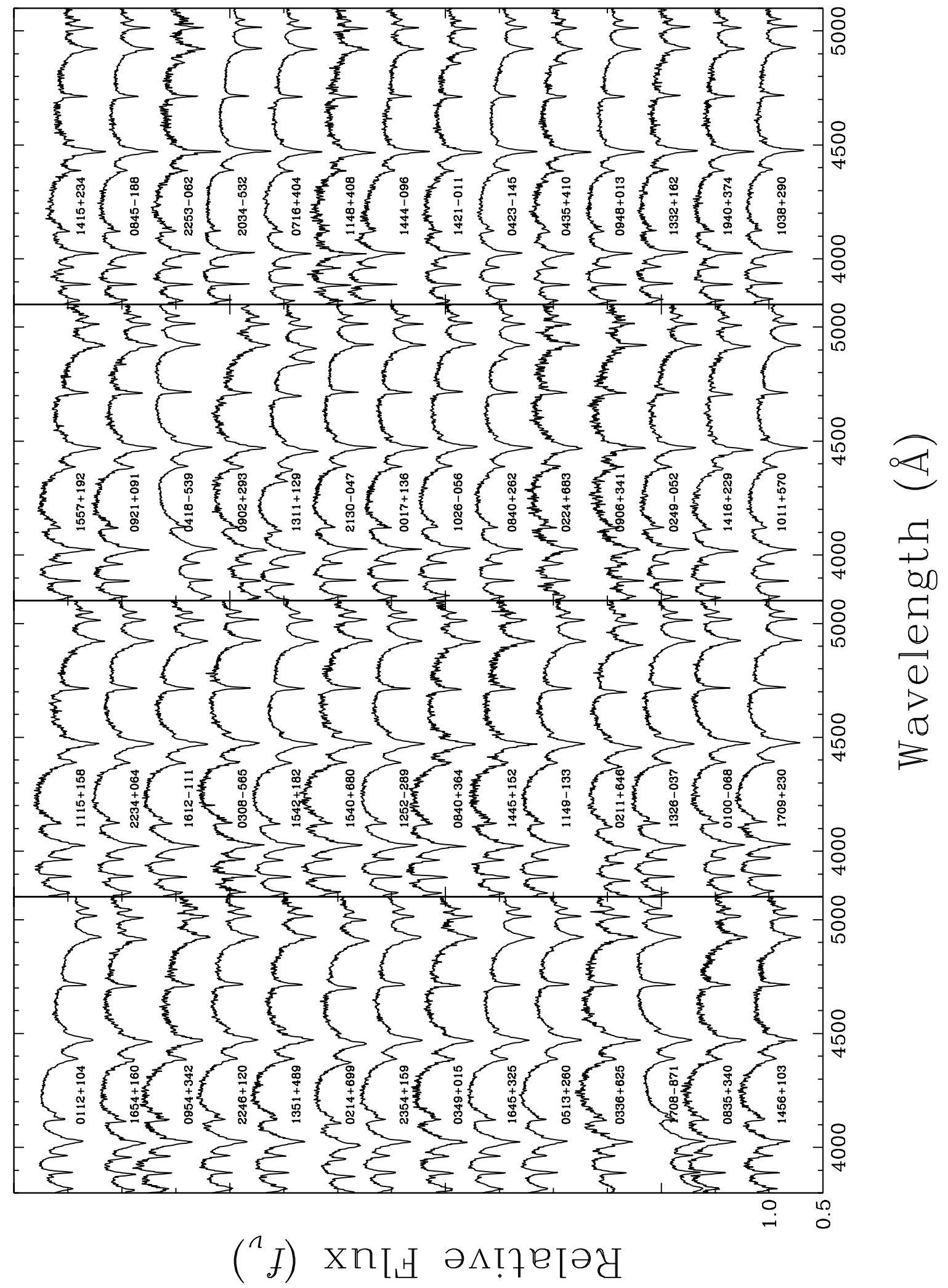

Figure $5 \mathrm{a}$ 


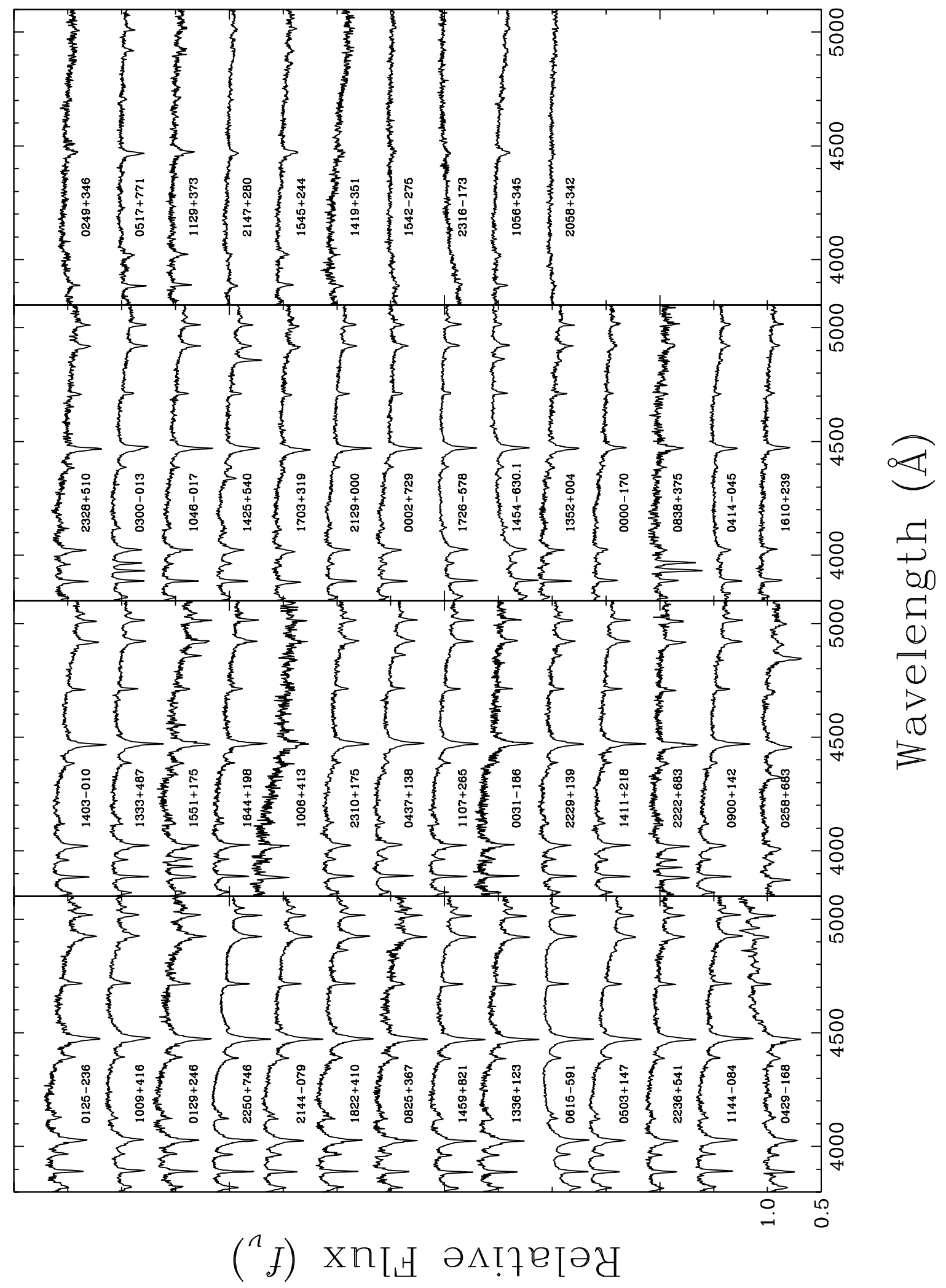

Figure 5b 


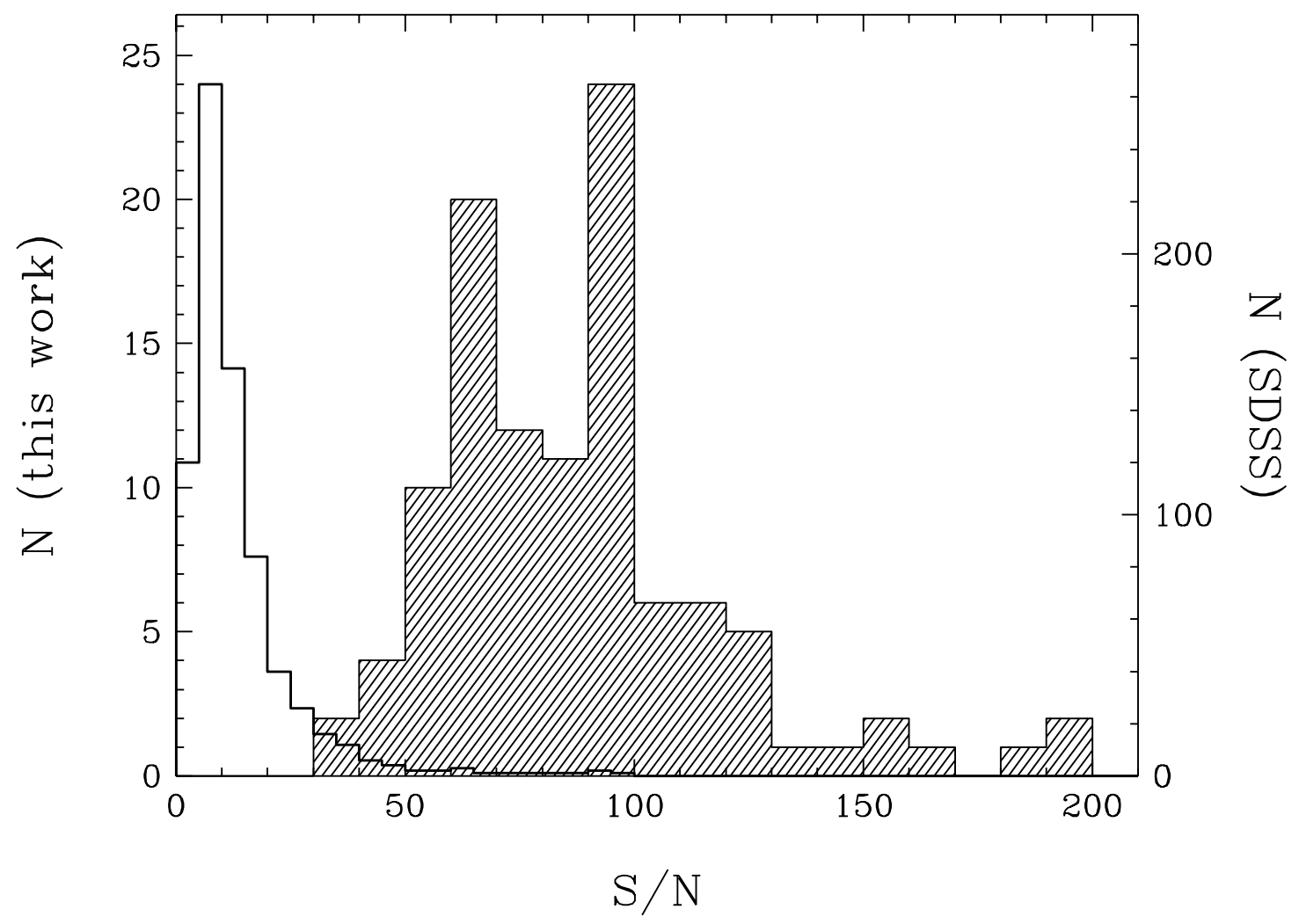

Figure 6 


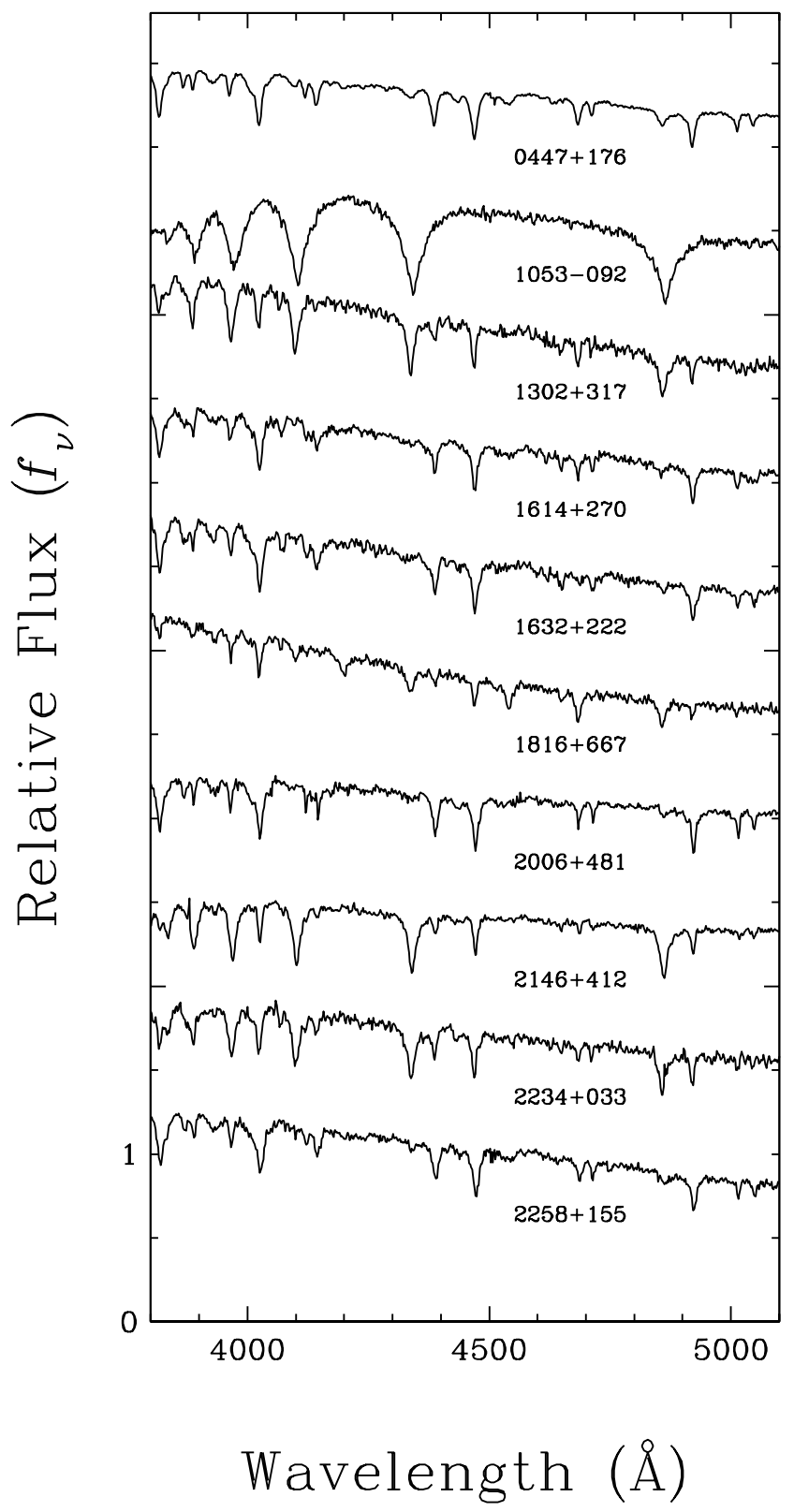

Figure 7 


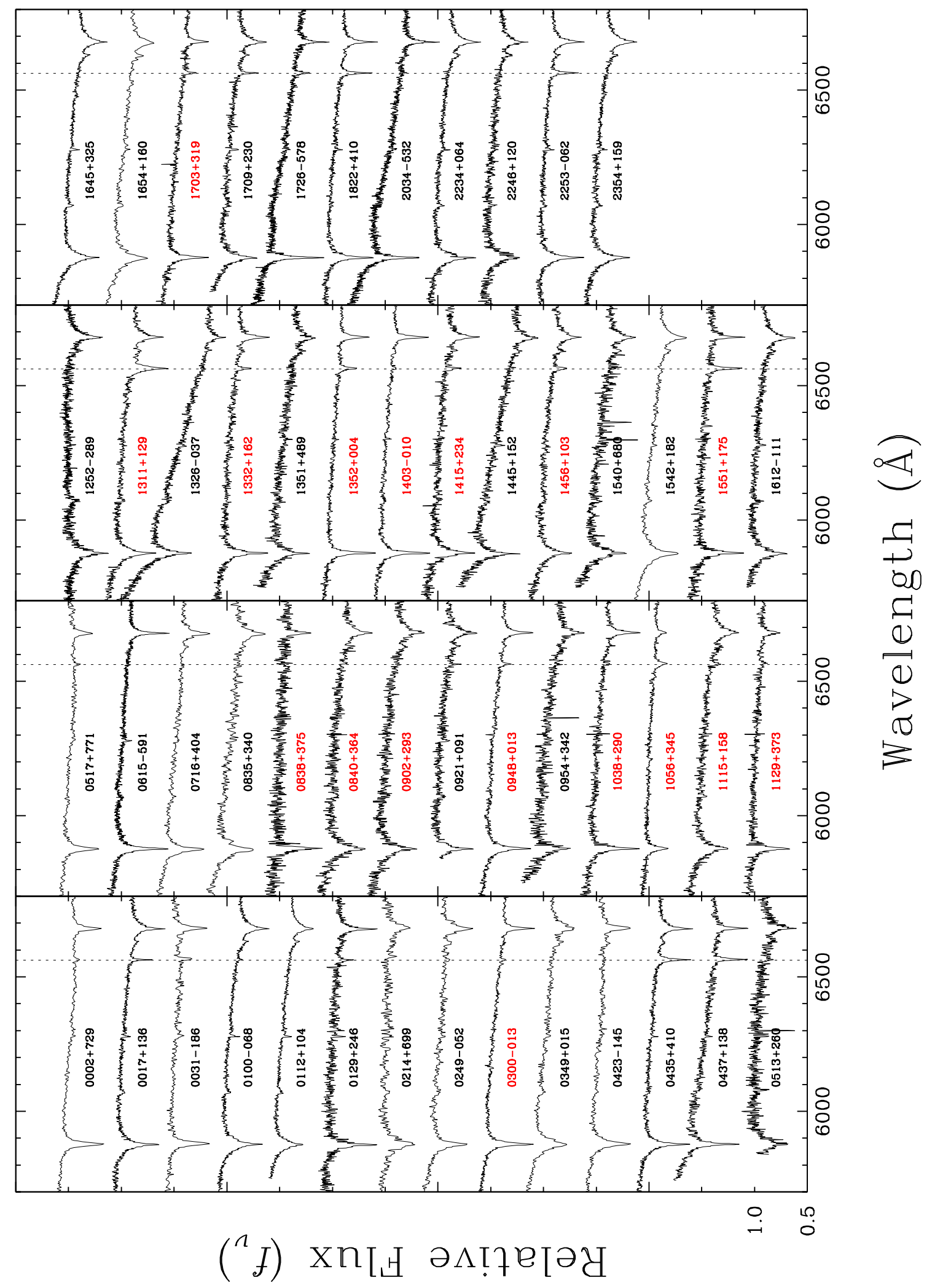

Figure 8 

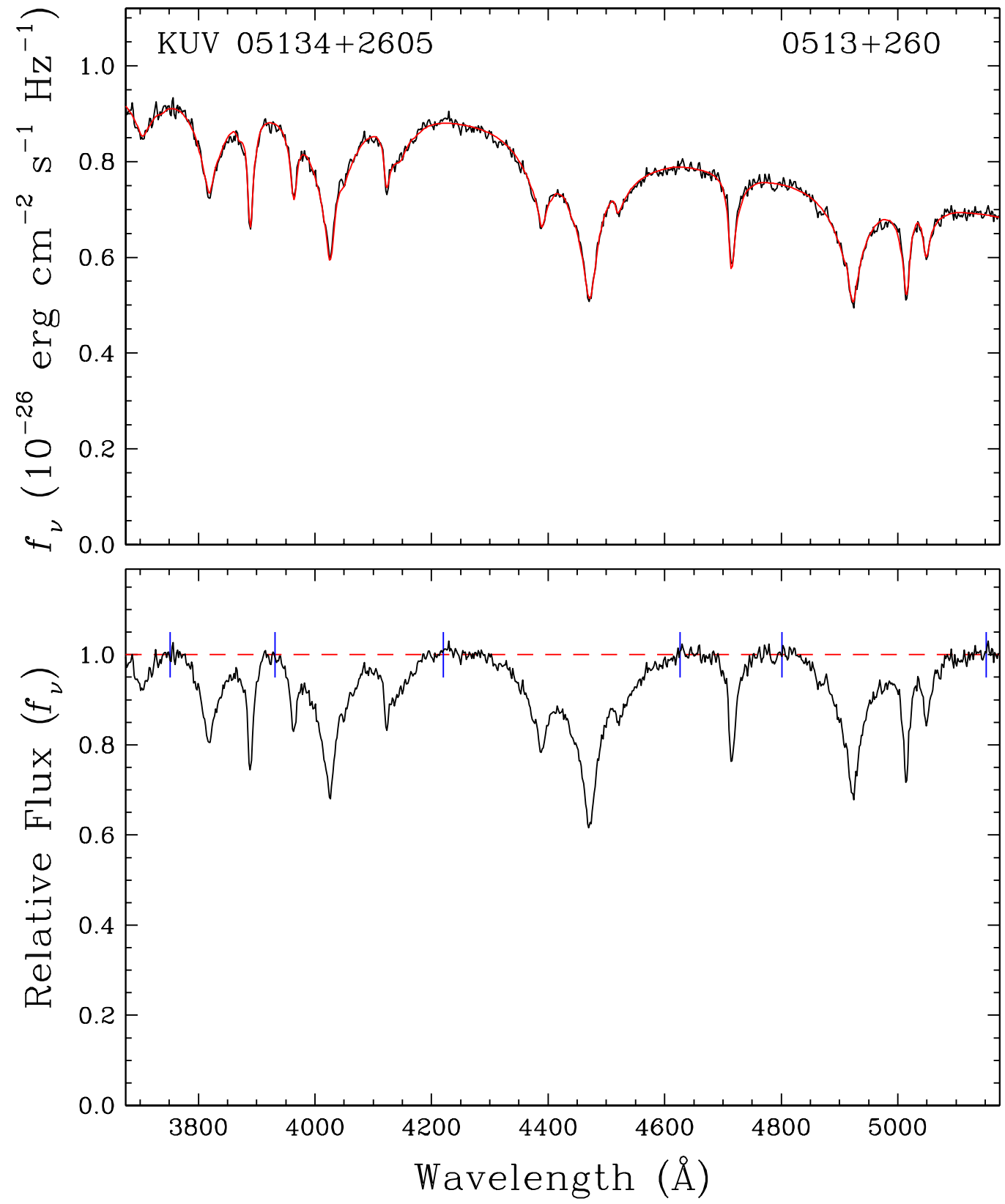

Figure 9 

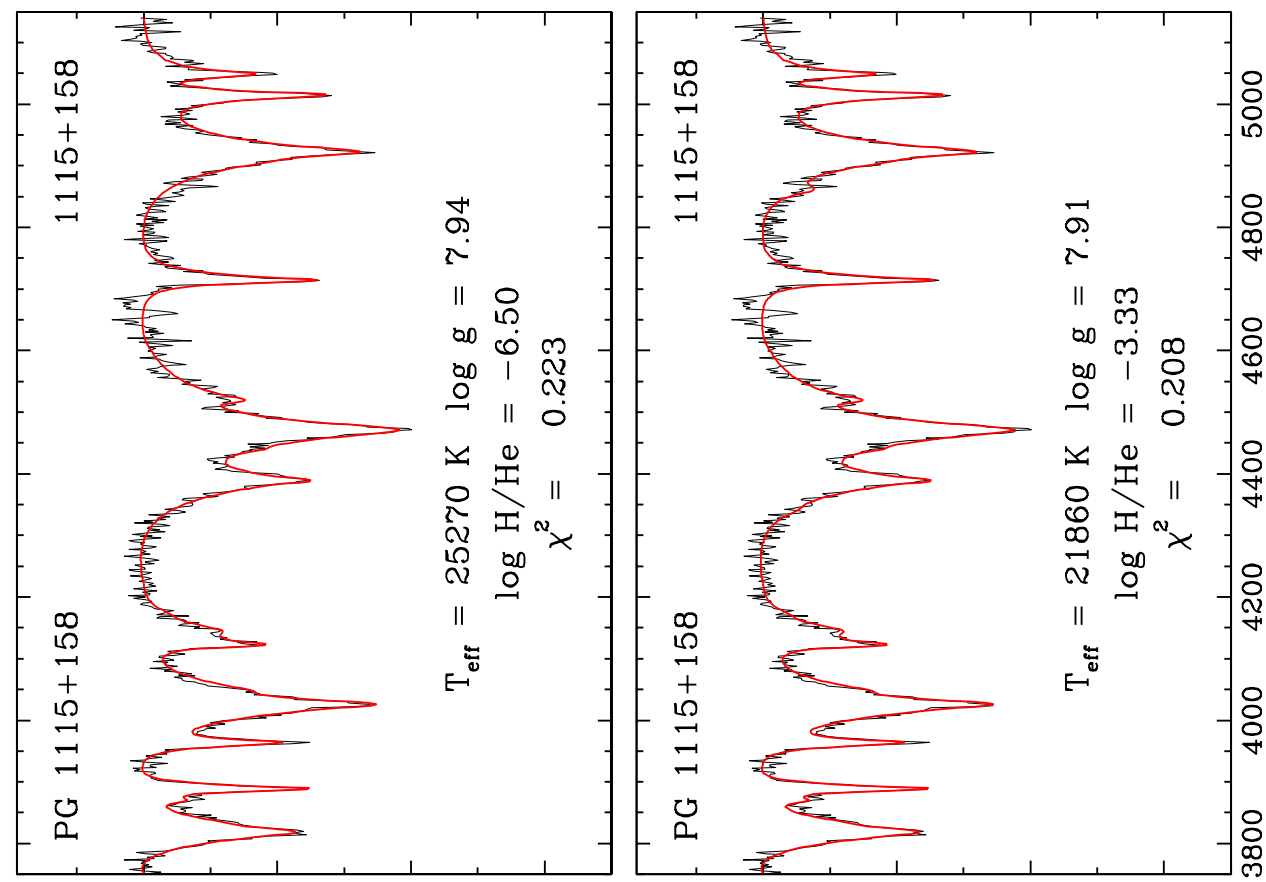

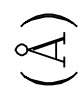
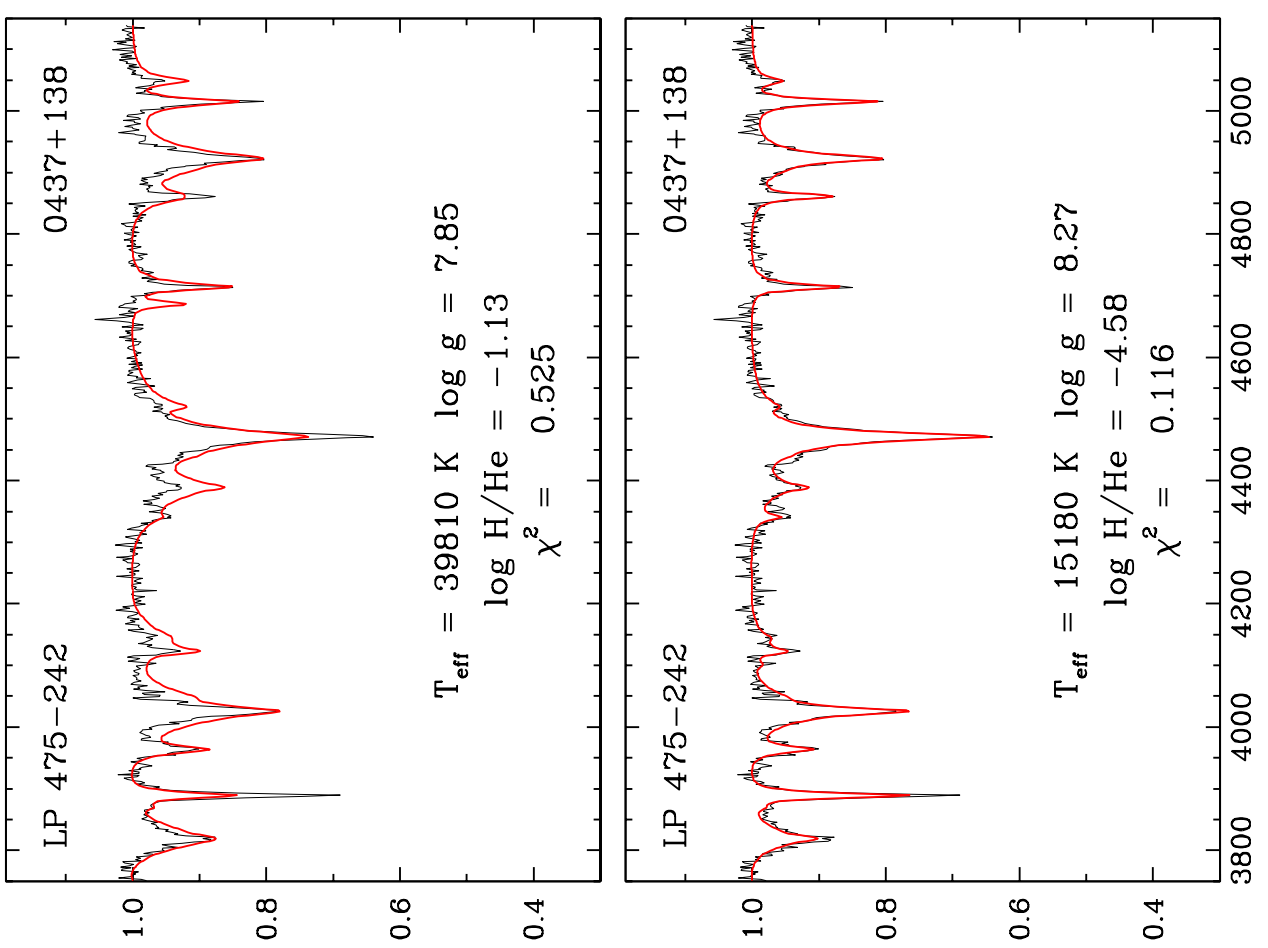

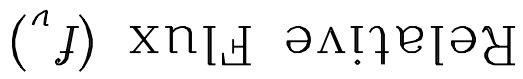

Figure 10 


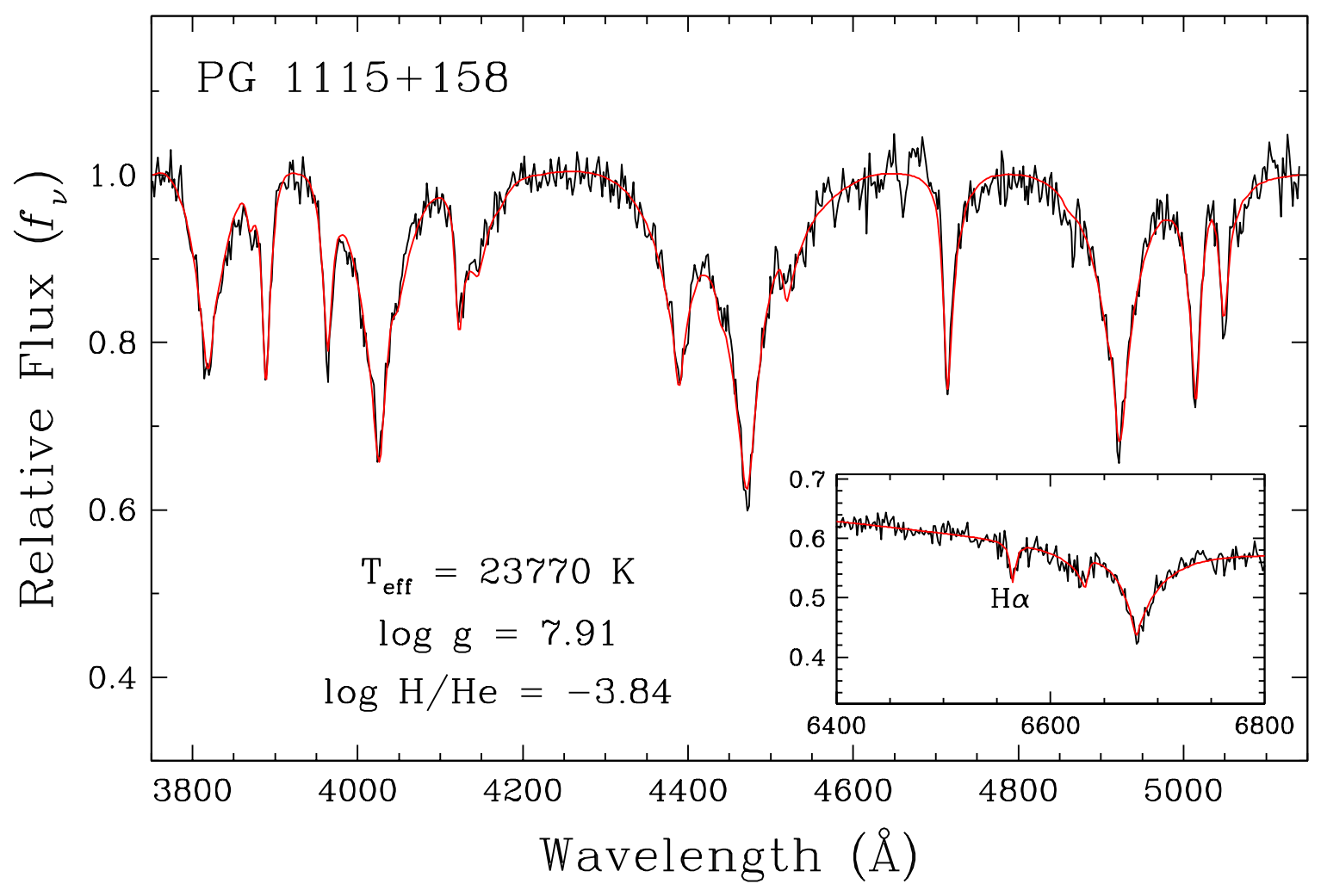

Figure 11 


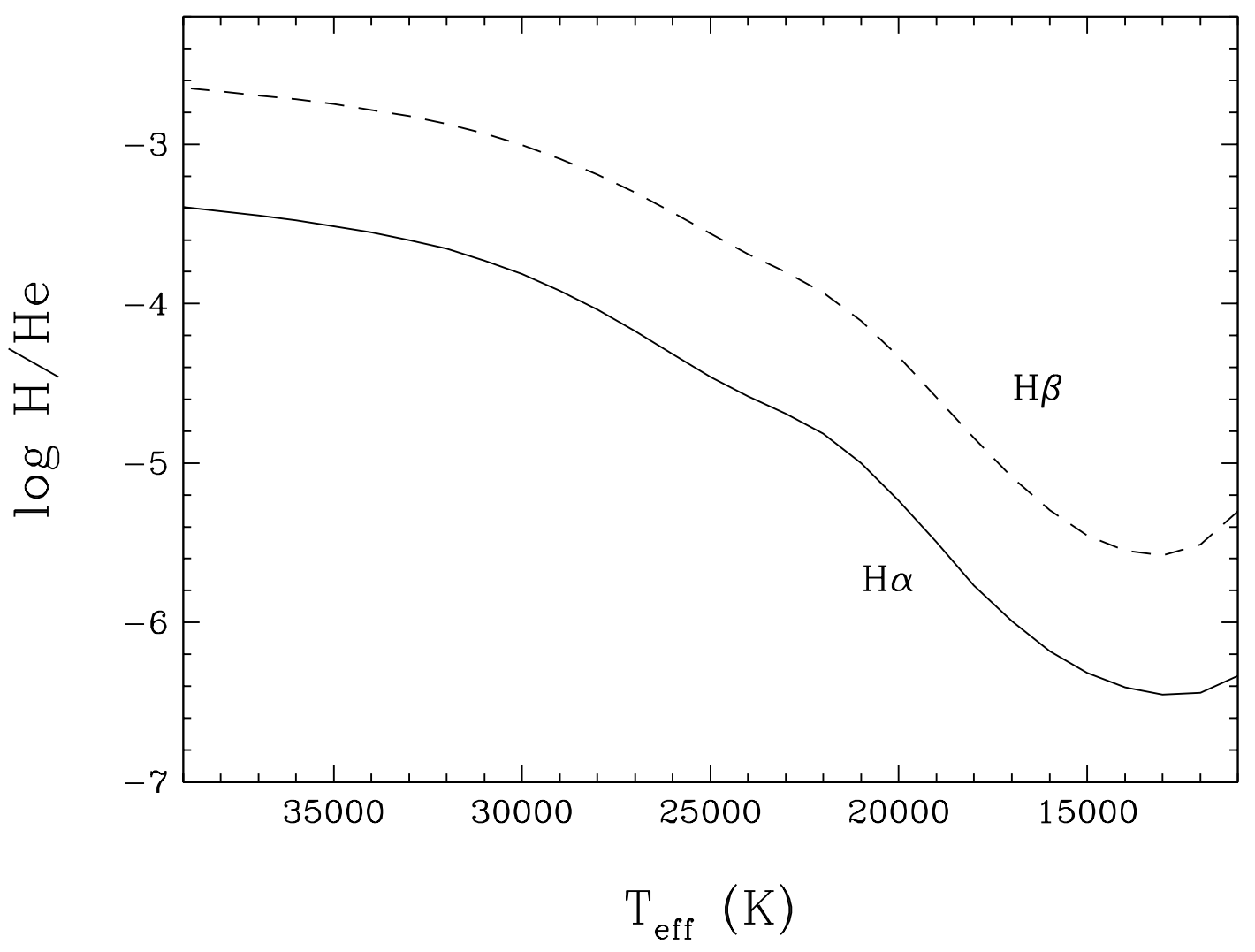

Figure 12 


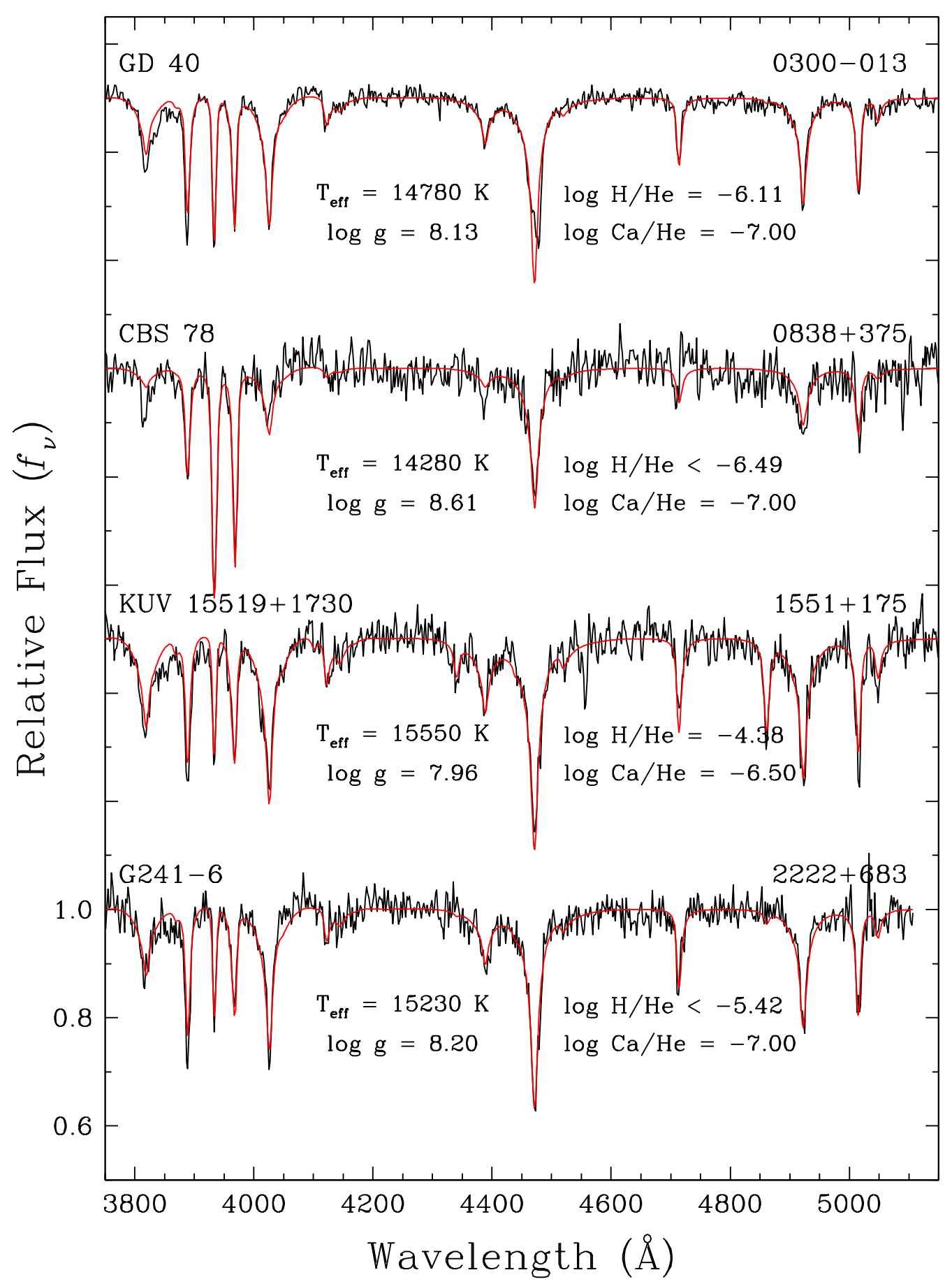

Figure 13 

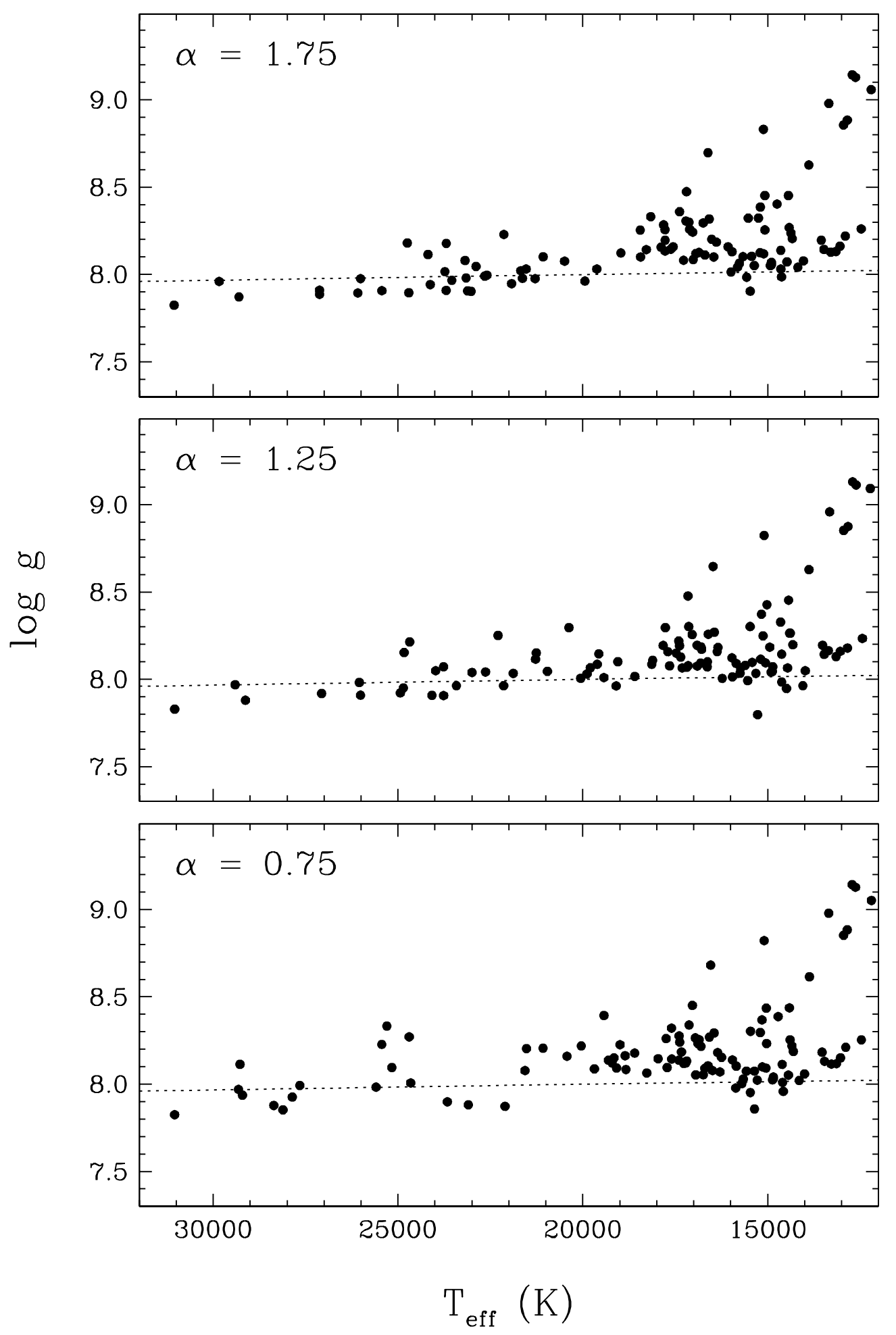

Figure 14 

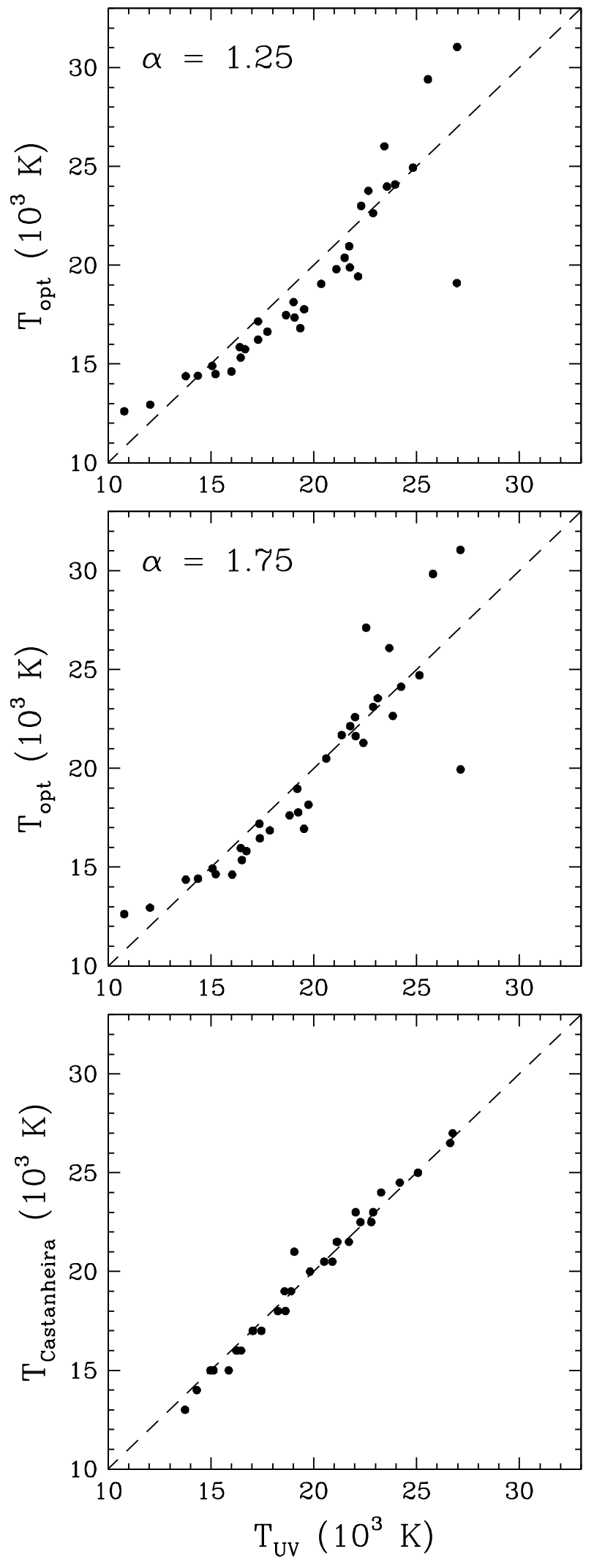

Figure 15 

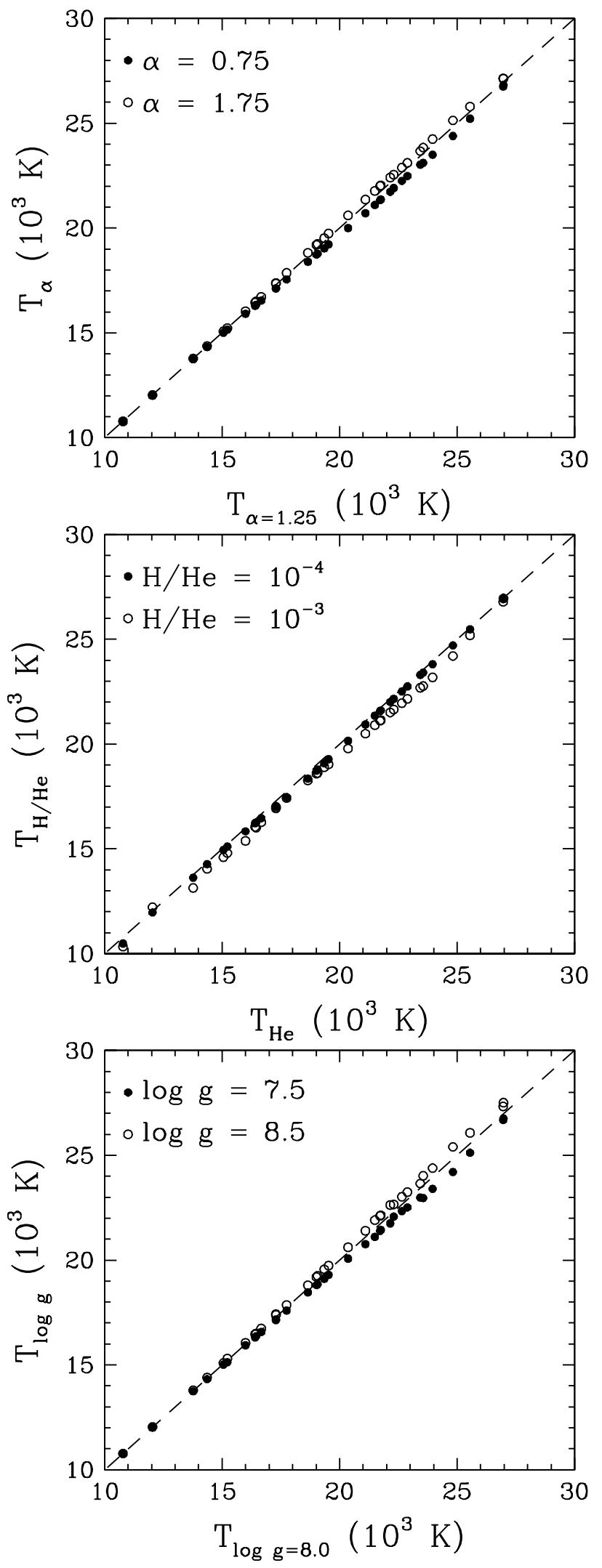

Figure 16 

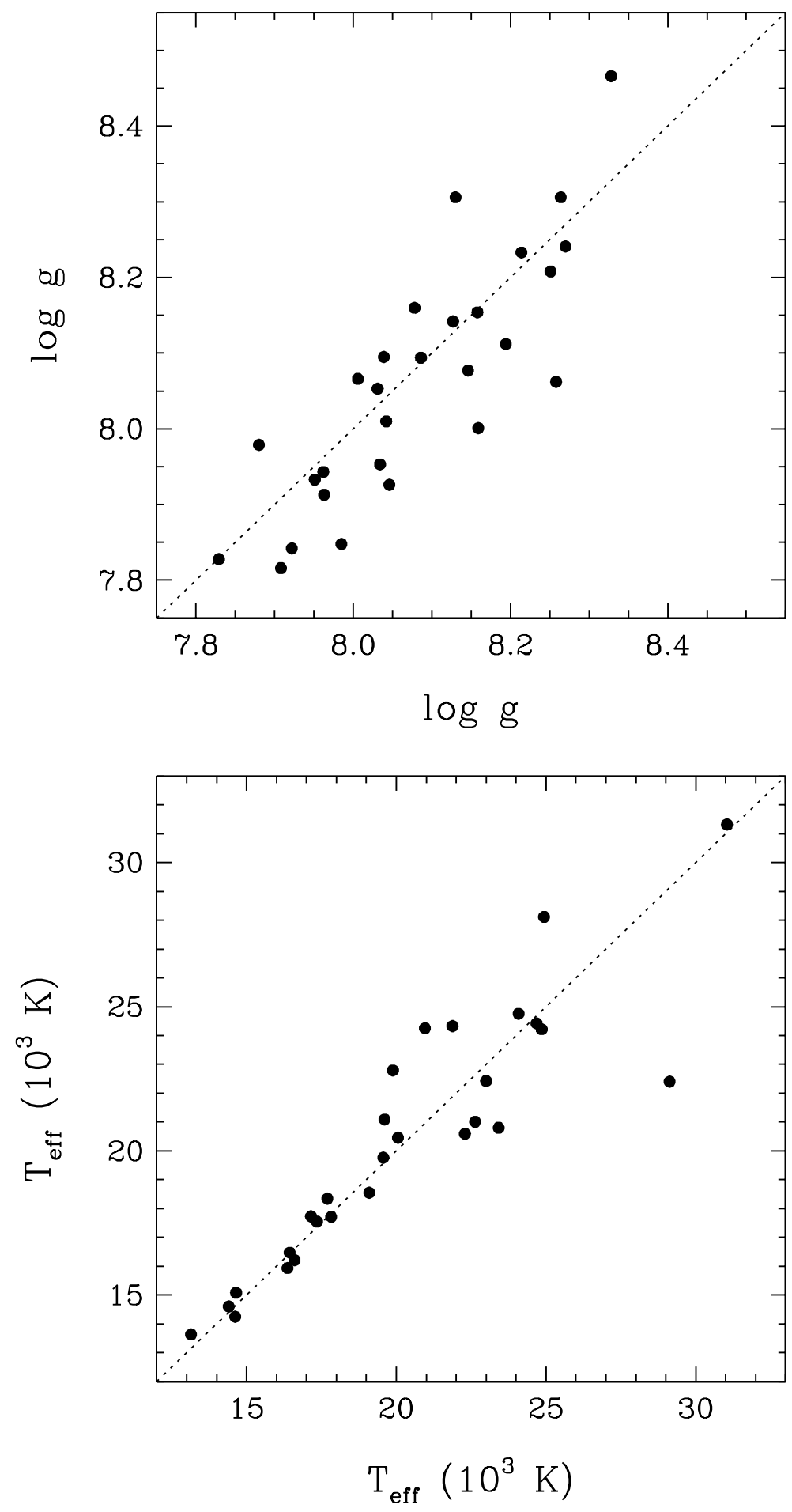

Figure 17 


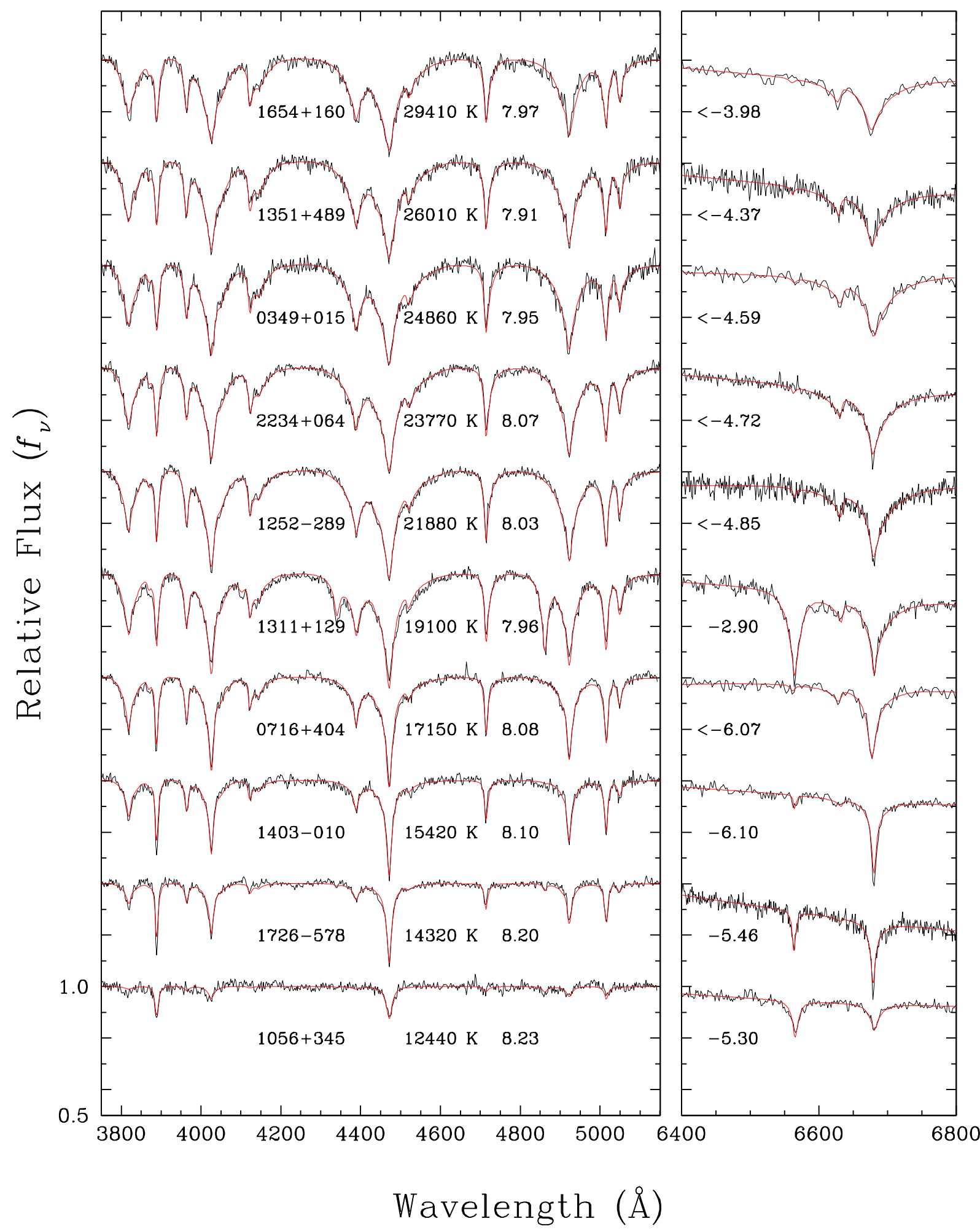

Figure 18 


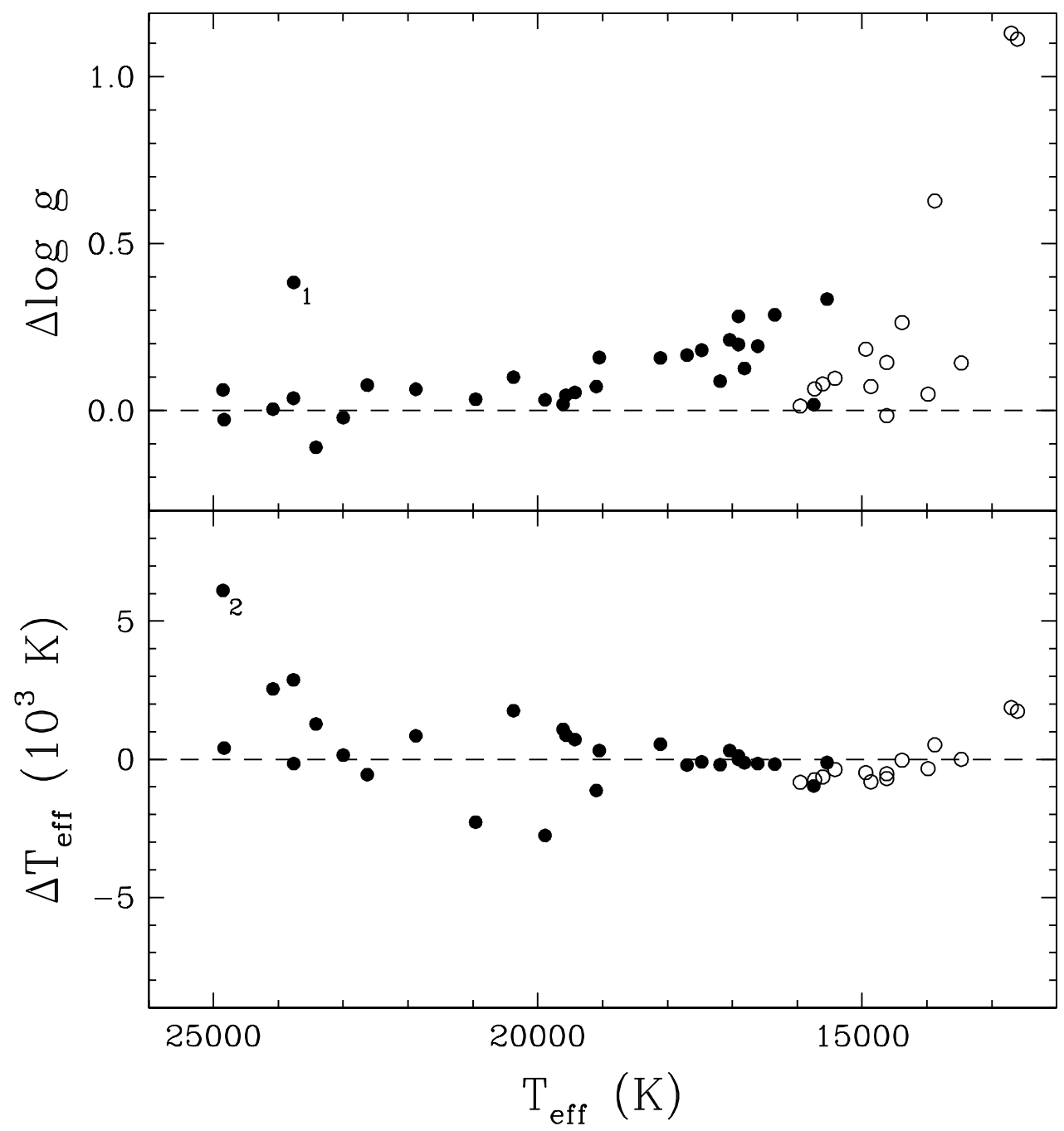

Figure 19 


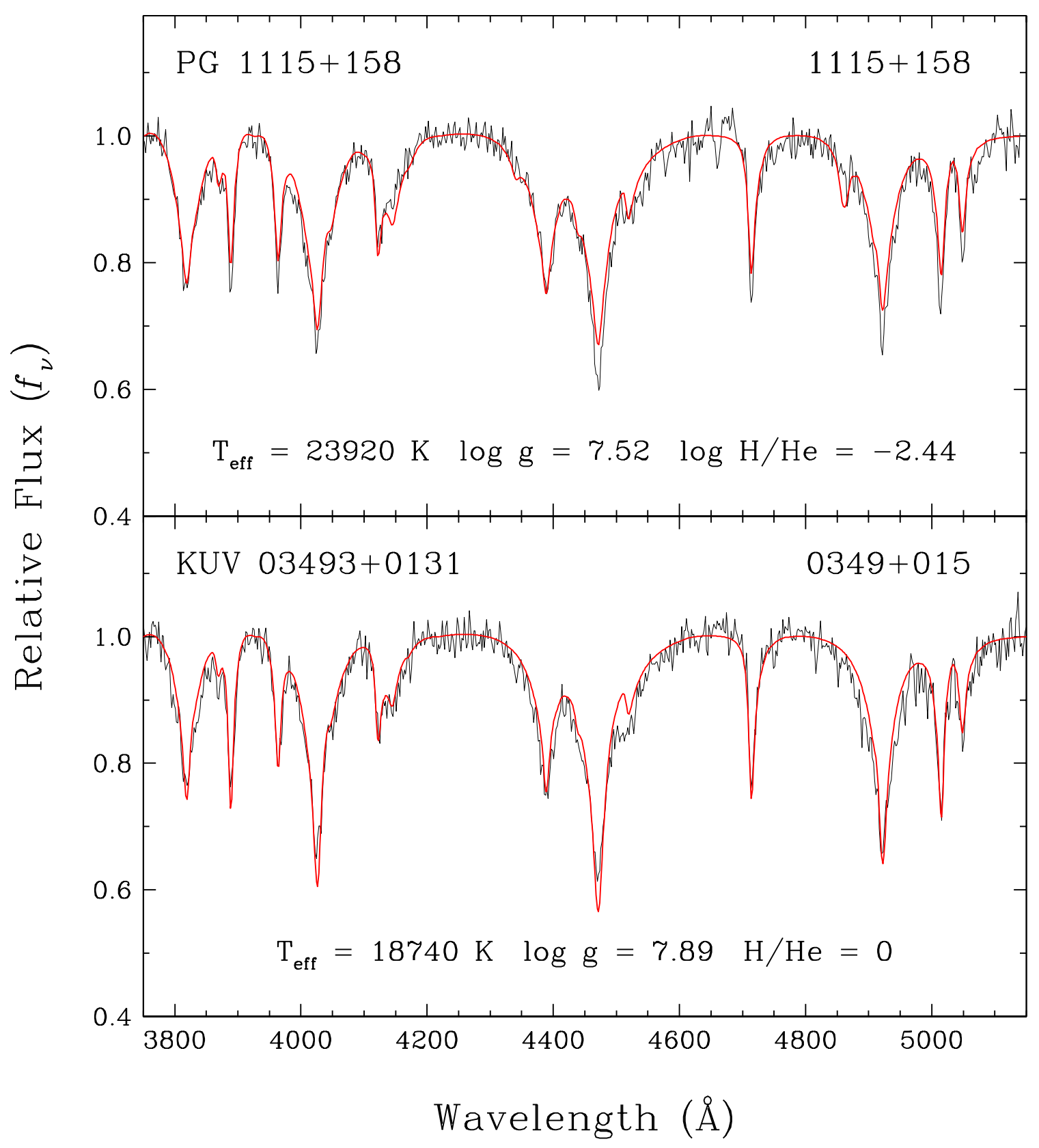

Figure 20 


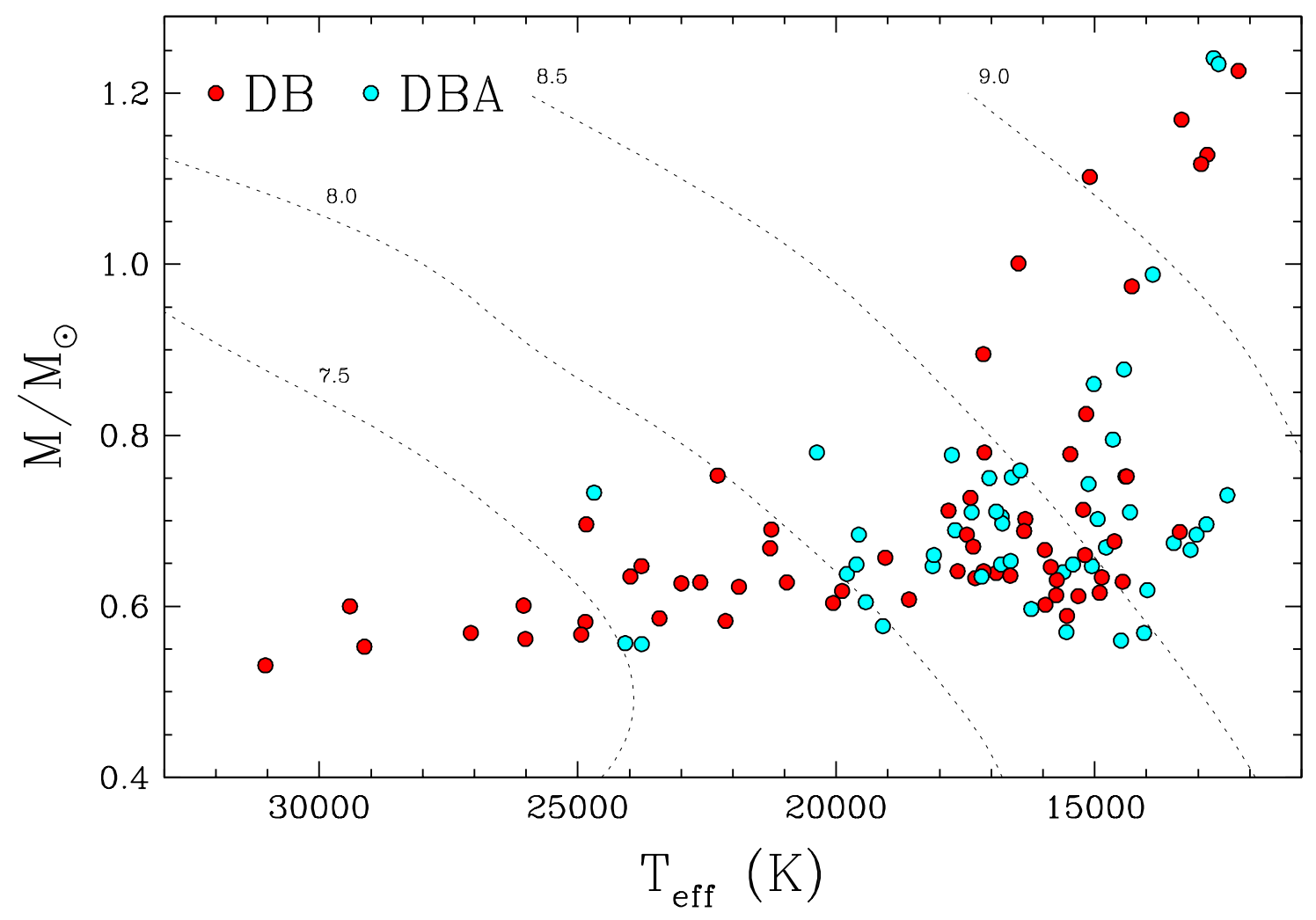

Figure 21 


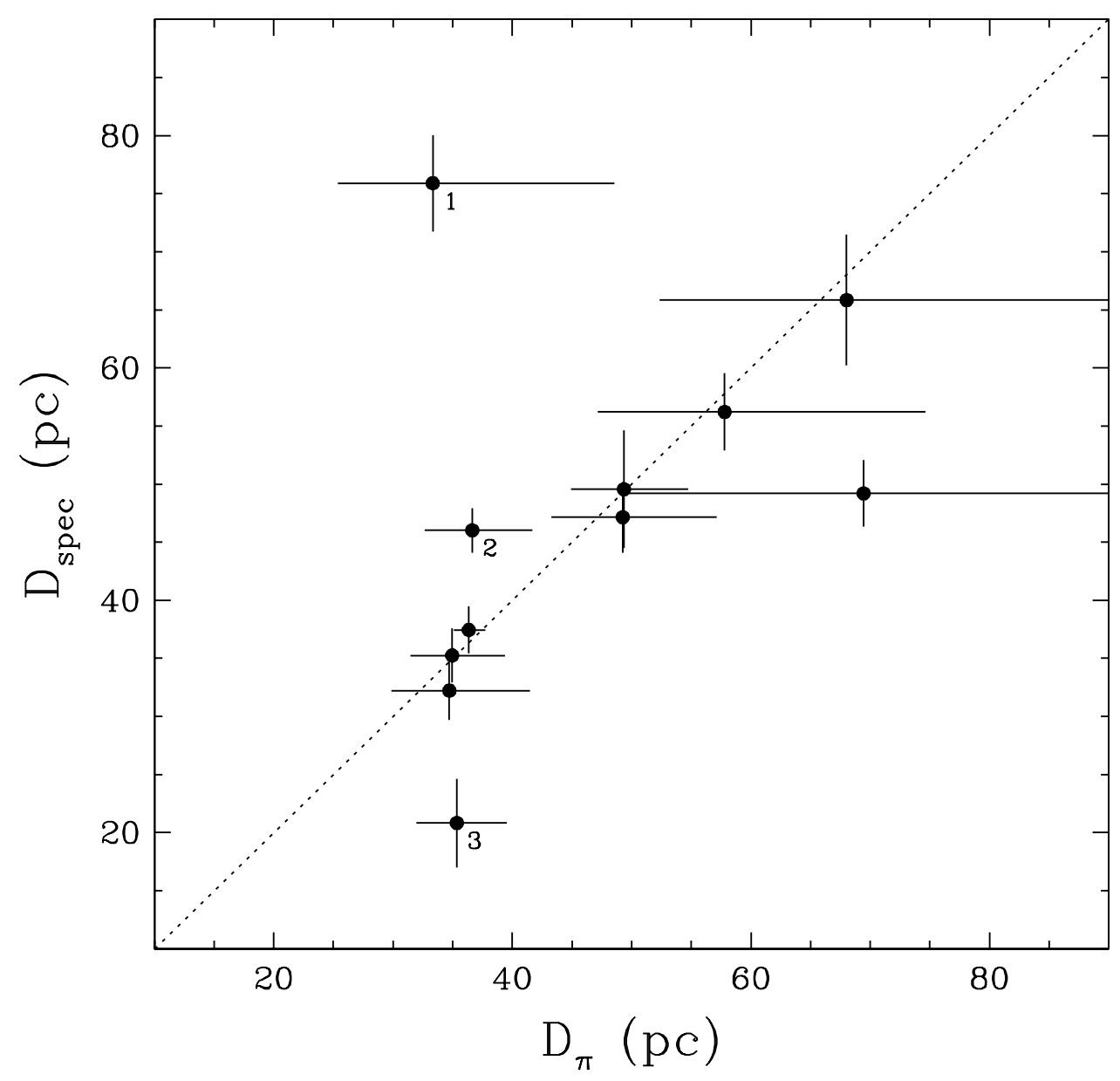

Figure 22 


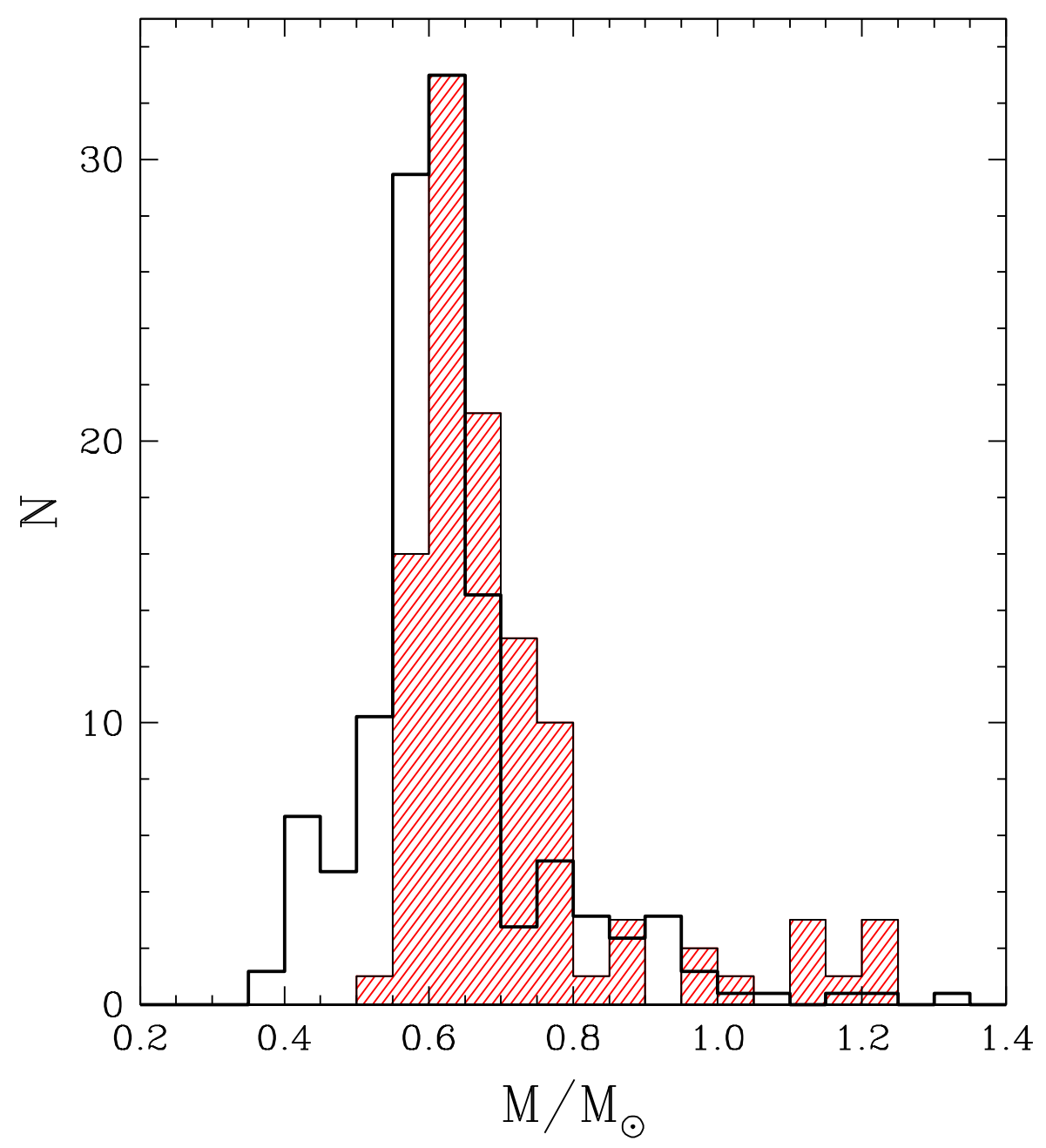

Figure 23 


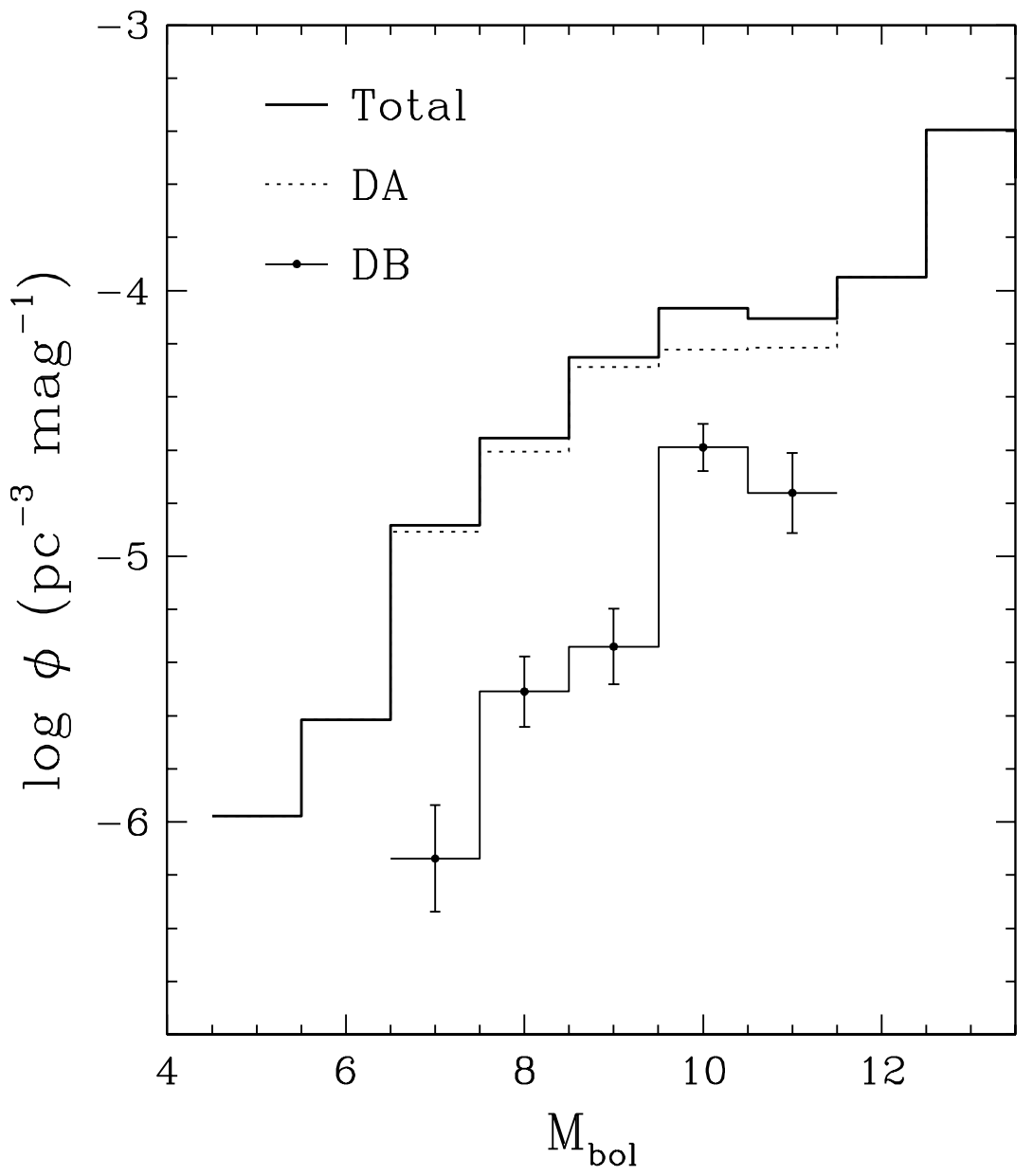

Figure 24 


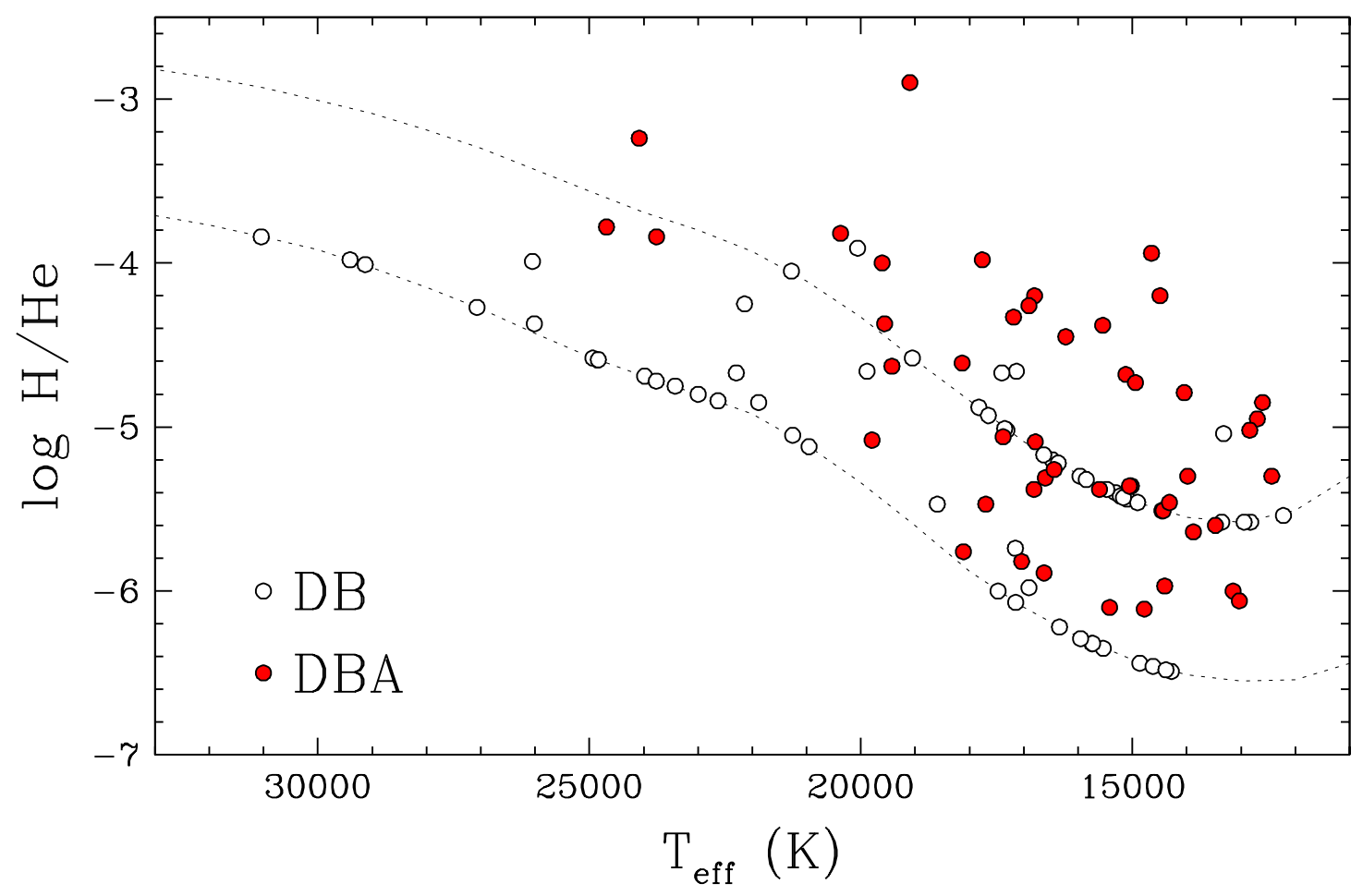

Figure 25 


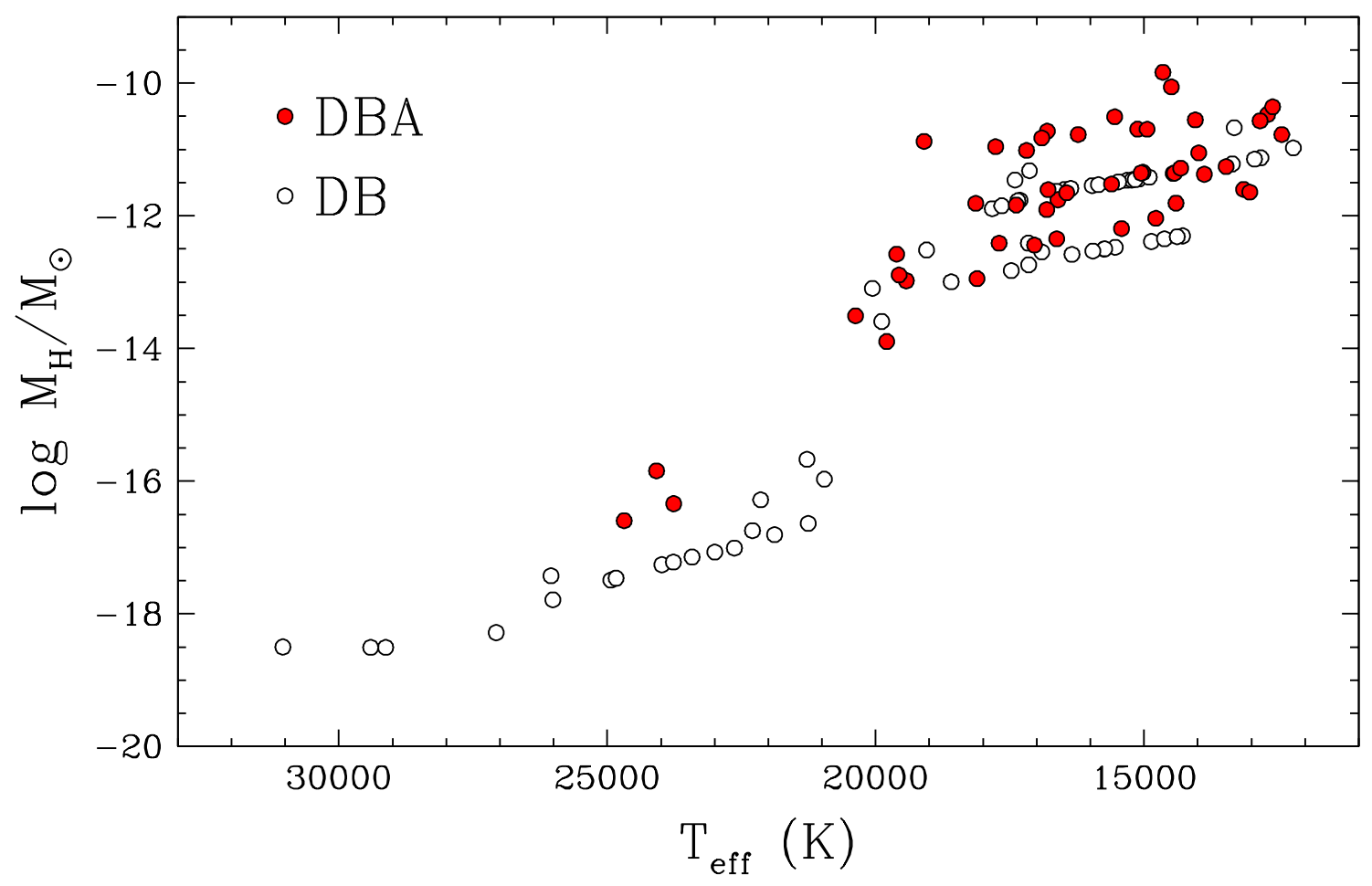

Figure 26 


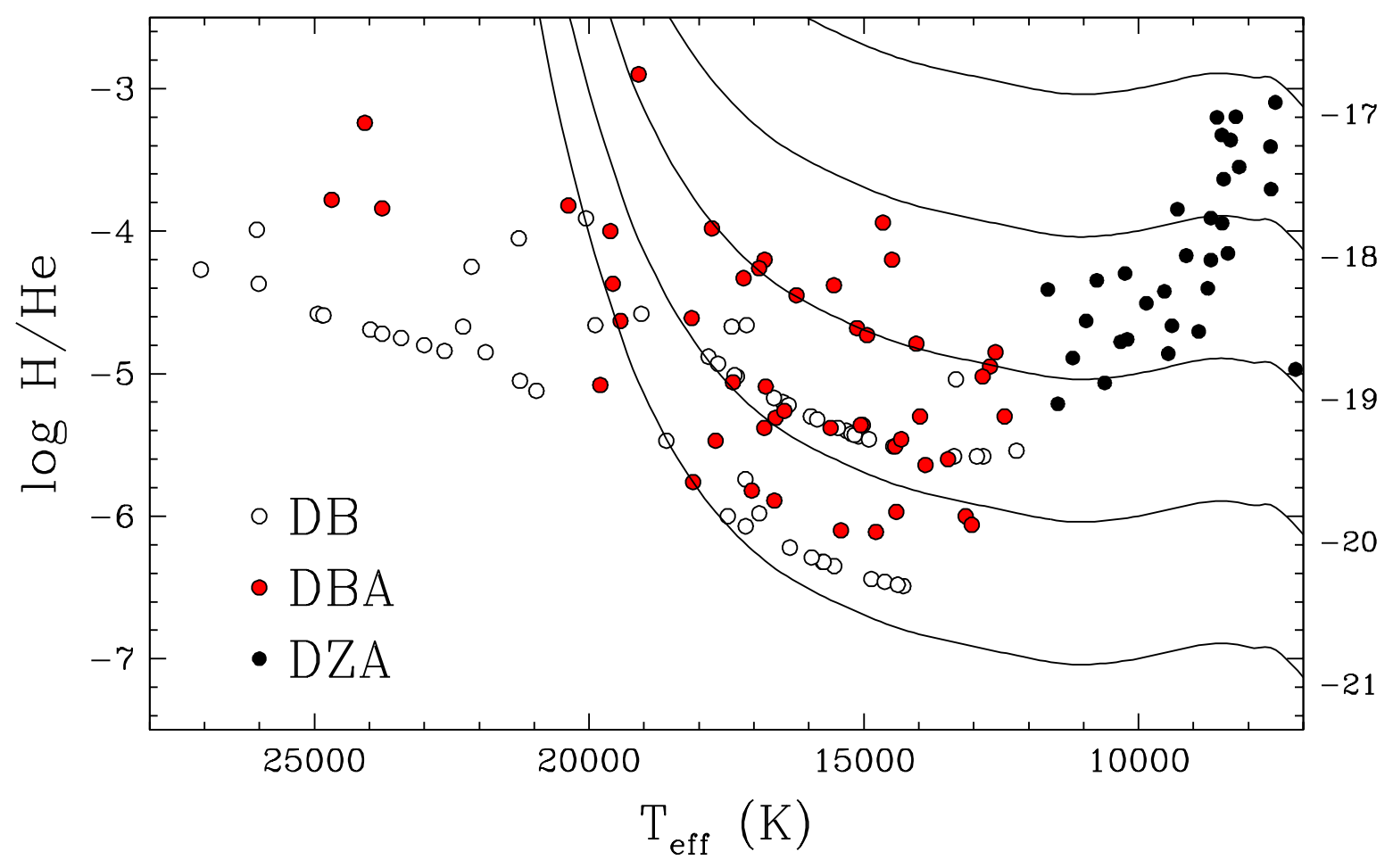

Figure 27 


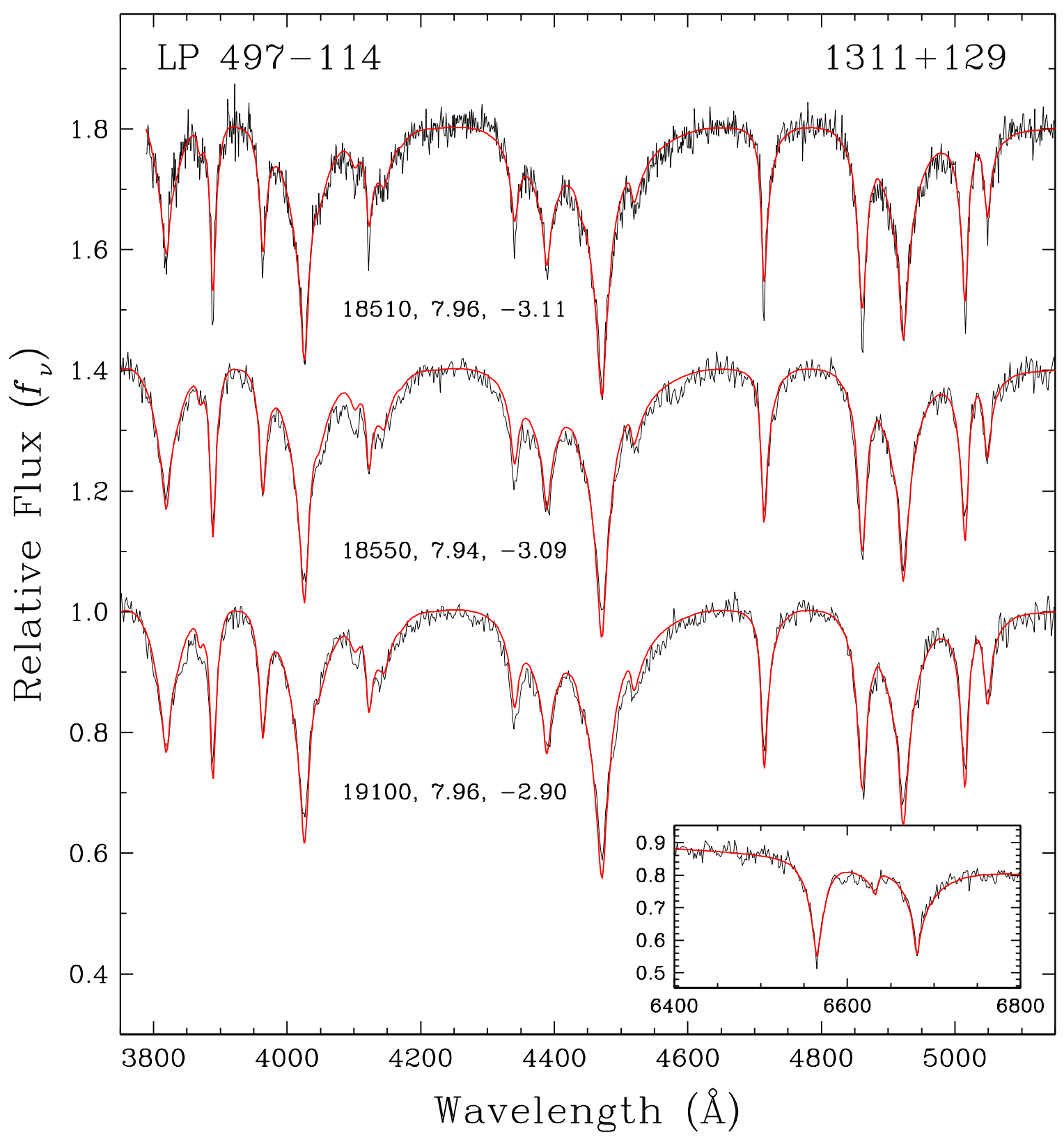

Figure 28 


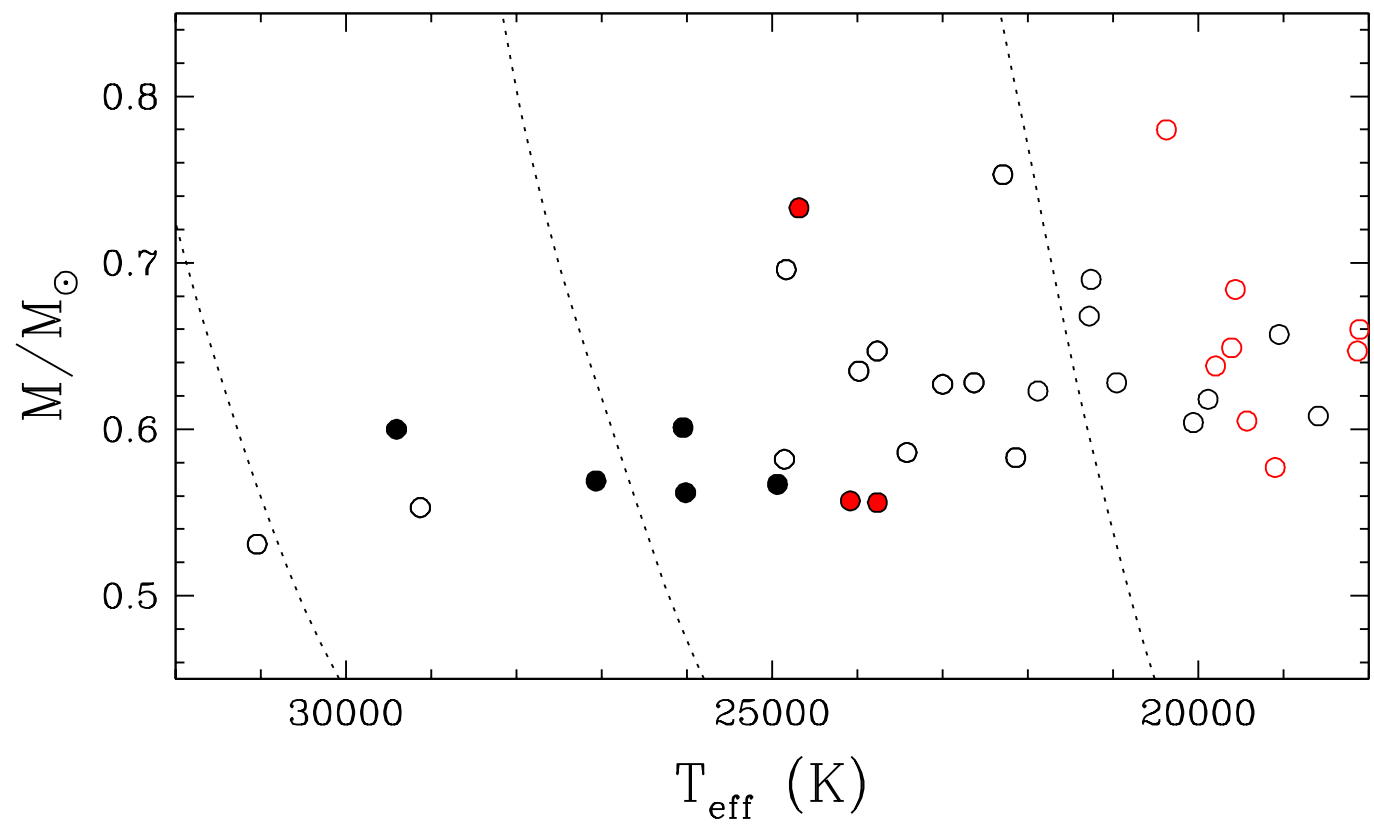

Figure 29 


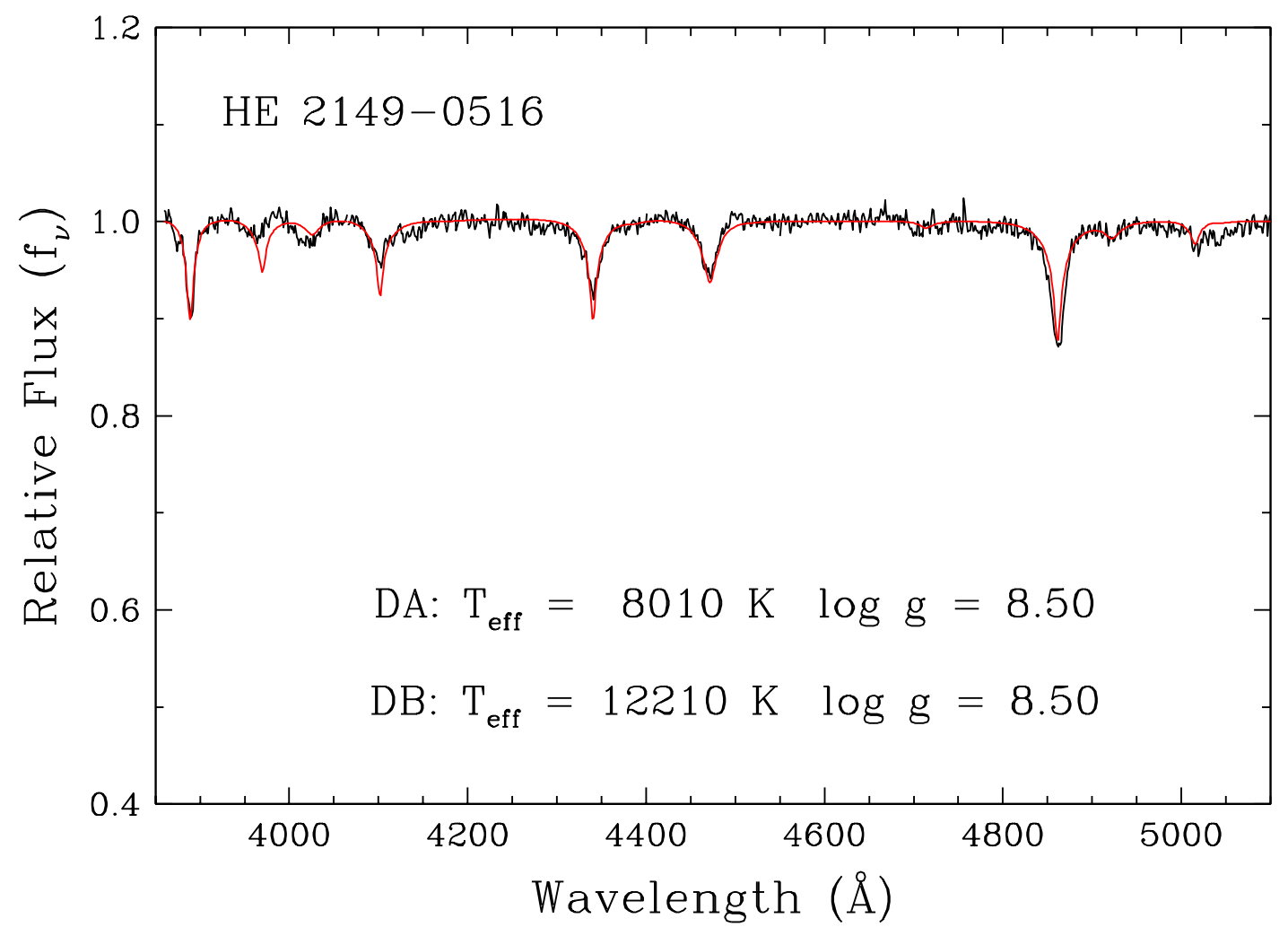

Figure 30 